PRRC 03-05

\title{
Risk Reduction with a Fuzzy Expert Exploration Tool
}

(Fourth Annual Technical Progress Report)

DOE Contract No. DE-AC-26-99BC15218

New Mexico Petroleum Recovery Research Center

New Mexico Institute of Mining and Technology

Socorro, NM 87801

(505) 835-5142

Date of Report:

Contract Date:

Anticipated Completion Date:

Award Amount for Current Fiscal Year:

Project Manager:

Principal Investigator:

Contributors:

Contracting Officer's Representative:

Reporting Period:
April 15, 2003

March 15, 1999

March 15, 2004

$\$ 420,000$

James Barnes, NPTO

Robert Balch

Ron Broadhead

William R. Mundorf, FETC

March 16, 2002 through March 15, 2003 


\section{Disclaimer}

This report was prepared as an account of work sponsored by an agency of the United States Government. Neither the United States Government nor any agency thereof, nor any of their employees, makes any warranty, express or implied, or assumes any legal liability or responsibility for the accuracy, completeness, or usefulness of any information, apparatus, product, or process disclosed, or represents that its use would not infringe privately owned rights. Reference herein to any specific commercial product, process, or service by trade name, trademark, manufacturer, or otherwise does not necessarily constitute or imply its endorsement, recommendation, or favoring by the United States Government or any agency thereof. The views and opinions of authors

expressed herein do not necessarily state or reflect those of the United States Government or any agency thereof. 


\begin{abstract}
Incomplete or sparse information on types of data such as geologic or formation characteristics introduces a high level of risk for oil exploration and development projects. "Expert" systems developed and used in several disciplines and industries have demonstrated beneficial results. A state-of-the-art exploration "expert" tool, relying on a computerized database and computer maps generated by neural networks, is being developed through the use of "fuzzy" logic, a relatively new mathematical treatment of imprecise or non-explicit parameters and values. Oil prospecting risk can be reduced with the use of a properly developed and validated "Fuzzy Expert Exploration (FEE) Tool."

This FEE Tool can be beneficial in many regions of the U.S. by enabling risk reduction in oil and gas prospecting as well as decreased prospecting and development costs. In the 1998-1999 oil industry environment, many smaller exploration companies lacked the resources of a pool of expert exploration personnel. Downsizing, low oil prices, and scarcity of exploration funds have also affected larger companies, and will, with time, affect the end users of oil industry products in the U.S. as reserves are depleted. The pool of experts is much reduced today. The FEE Tool will benefit a diverse group in the U.S., leading to a more efficient use of scarce funds, and possibly decreasing dependence on foreign oil and lower product prices for consumers.
\end{abstract}

This fourth of five annual reports contains a summary of progress to date, problems encountered, plans for the next year, and an assessment of the prospects for future progress. The emphasis during the April 2002 through March 2003 period was directed toward Silurian-Devonian geology, development of rules for the fuzzy system, and on-line software. 


\section{TABLE OF CONTENTS}

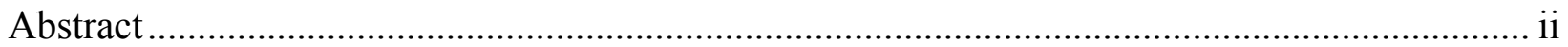

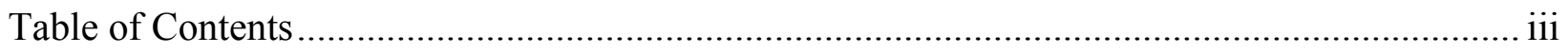

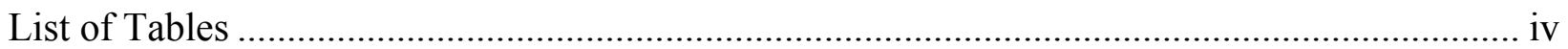

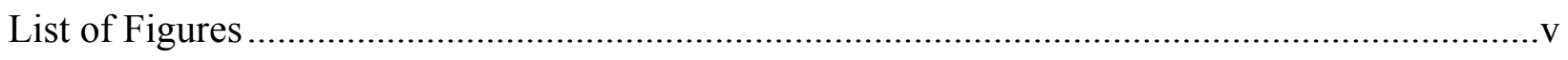

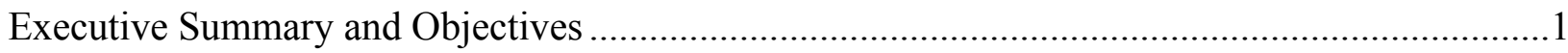

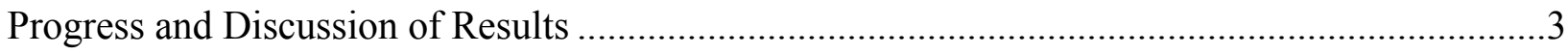

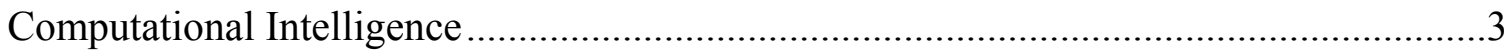

Web-Based Data Management System (WDMS) ....................................................28

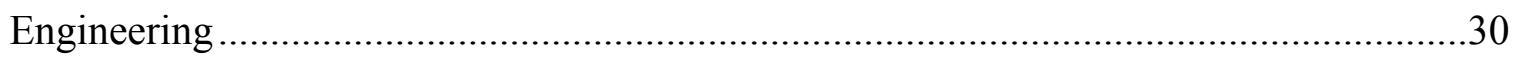

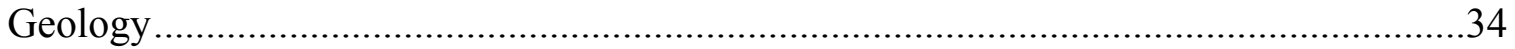

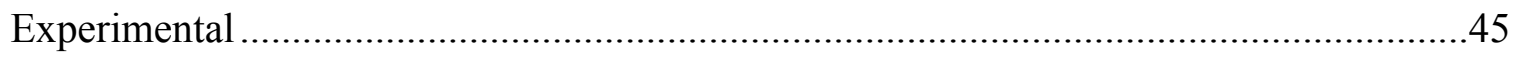

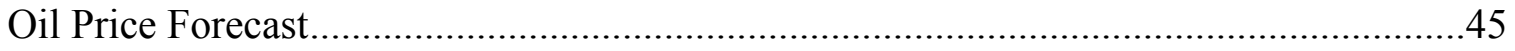

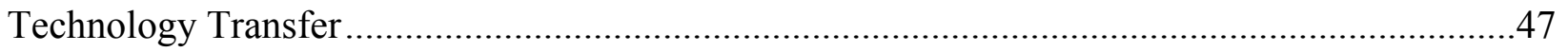

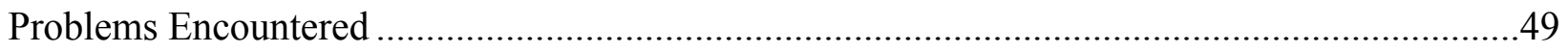

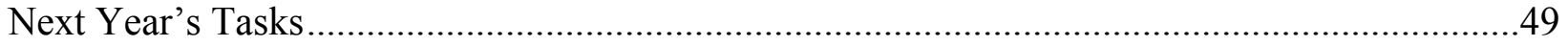

Continuing Expert System Development...........................................................49

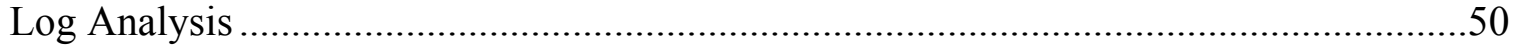

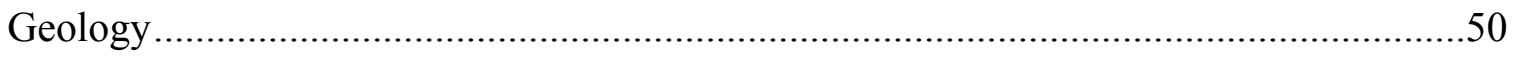

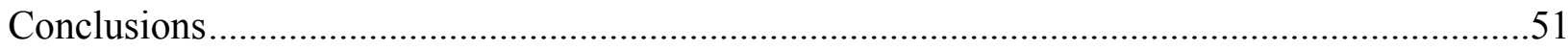

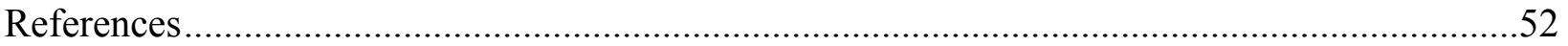




\section{LIST OF TABLES}

Table 1. Variables Selected as Optimal ..............................................................................

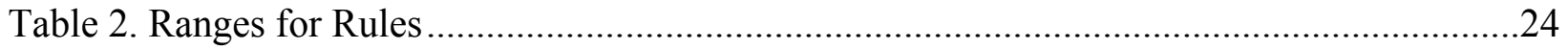

Table 3. Numerical Values Generated Using Trap and Source Rules ......................................25

Table 4. Results of "gaussmf” Function Trial ......................................................................26

Table 5. Numerical Results from Psinmf/dsinmf, pimf Functions .........................................27

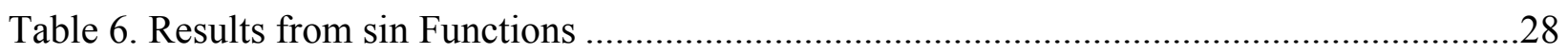

Table 7. Goodness of the Relationship between Commodity Future Price and Oil Price............47 


\section{LIST OF FIGURES}

Fig. 1. Histogram of average hydrocarbon equivalent. ........................................................53

Fig. 2. Log filtered version of histogram from Fig. 1. .........................................................53

Fig. 3. Sample fuzzy membership functions drawn for two points of a 100-point random data distribution.

Fig. 4. Sample fuzzy membership functions drawn for two points of a 100-point random data distribution with a trend added.

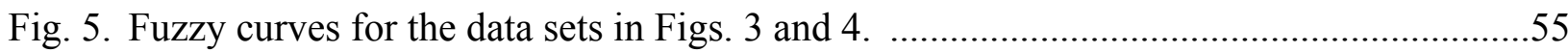

Fig. 6. Operation of second stage fuzzy ranking program FuzzyOnline. ...................................56

Fig. 7. Crossplot of neural network training using the inputs identified via FuzzyOnline...........57

Fig. 8. Crossplot of testing data for the trained neural network of Fig. 7 . ................................57

Fig. 9. Map of predicted production potential based on the trained and tested neural network regression.

Fig. 10. Benefits of neural network regressions for complex problems where forecasts need to be made.

Fig. 11. The original schematic for the fuzzy expert system shell.

Fig. 12. More complicated system, which breaks the analysis into several separate categories to simplify calculations and customization

Fig. 13. Initial rules for trap assessment (Part I)............................................................61

Fig. 14. Initial rules for trap assessment (Part II). ..........................................................61

Fig. 15. Initial rules for trap assessment (Part III) ..........................................................62

Fig. 16. Initial rules for source rock assessment (Part I). ...............................................62

Fig. 17. Initial rules for source rock assessment (Part II)...............................................63

Fig. 18. Initial rules for source rock assessment (Part III) ..............................................63

Fig. 19. Initial rules for source rock assessment (Part IV)....................................................64

Fig. 20. Initial rules for source rock assessment (Part V).....................................................64 
Fig. 21. Initial rules for regional assessment (Part I)

Fig. 22. Initial rules for regional assessment (Part II). .65

Fig. 23 Histogram showing the skewed nature and large mean of the thickness of the porous sand. .66

Fig. 24. Histogram of the differences in initial and enhanced estimate when thickness enhancement reduced. $($ Difference $=$ final - initial $)$ .66

Fig. 25. Fuzzy membership functions for total organic carbon. .67

Fg. 26. Fuzzy membership functions for porosity. .67

Fig. 27. Fuzzy membership curves for the trapmf function. .68

Fig. 28. Summary of all ranges for Trap (left), Regional (middle), and risk (right) as defined in Table 2. .68

Fig. 29. Fuzzy membership curves for the gaussmf function.... 69

Fig. 30. Fuzzy membership curves for the gauss $2 \mathrm{mf}$ function. .69

Fig. 31. Fuzzy membership curves for a composite of zmf and smf functions. .70

Fig. 32. Plot of membership curves with "dsinmf" and "psinmf" used for both low and medium and the "zmf" and "smf" functions for the zero and high. .70

Fig. 33. Plot of membership curves when the "pimf" function is used for low and medium parameters and the "zmf" and "smf" functions for zero and high.

Fig. 34. Plot of membership curves using "sin" curves for Low and Medium and using "zmf" and "smf" curves for the zero and high parameters.

Fig. 35. Overtraining cartoon demonstrates that large errors are possible with $100 \%$ correlation coefficient.

Fig. 36. Small core dataset trains to almost $100 \%$ correlation coefficient. .72

Fig. 37. Training to almost $100 \%$ correlation coefficient produces an extremely poor blind test. .73

Fig. 38. Typical logs and core measurements through $200 \mathrm{ft}$ of an algal mound reservoir. .73

Fig. 39. Shallow resistivity log fuzzy curve. .74 
Fig. 40. Gamma ray log fuzzy curve. .74

Fig. 41. Trained neural network BVO; pseudo-log vs. core measurements. .............................75

Fig. 42. Trained neural network BVG; pseudo-log vs. core measurements. ............................75

Fig. 43. Trained neural network BVW; pseudo-log vs. core measurements. ............................76

Fig. 44. Trained neural network aspect ratio; pseudo-log vs. core measurements. .....................76

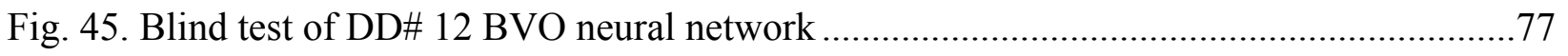

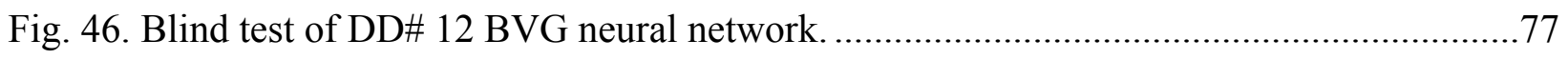

Fig. 47. Sum of pseudo-log BVO; $\log$ predictions vs. initial oil rate. ....................................78

Fig. 48. Sum of pseudo-log BVG; $\log$ predictions vs. initial gas rate....................................78

Fig. 49. Sum and average neural network predictions vs. actual initial oil rate.........................79

Fig. 50. Sum and average neural network predictions vs. actual initial gas rate........................79

Fig. 51. Location of project area in relation to Delaware Basin and other geologic elements, Permian Basin area southeast New Mexico and west Texas............................80

Fig. 52. Stratigraphic chart of Delaware Mountain Group.....................................................8

Fig. 53. Location of well data control points used in the Brushy Canyon part of this project. See Fig. 511 for map location.

Fig. 54. Three-dimensional view of structure on Bone Spring Formation and location of oil pools (in green) productive from the lower part of the Brushy Canyon Formation

Fig. 55. Three-dimensional view of structure on lower Brushy Canyon Formation and location of oil pools (in green) productive from the lower part of the Brushy Canyon Formation .82

Fig. 56. Isopach map of lower Brushy Canyon Formation.

Fig. 57. Areas productive from lower Brushy Canyon, net thickness of lower Brushy Canyon sandstones with porosity $>15 \%$, and wells that unsuccessfully tested the lower Brushy Canyon.

Fig. 58. Areas productive from lower Brushy Canyon and net thickness of lower Brushy Canyon sandstones with porosity $>10 \%$. .83 
Fig. 59. Net thickness of lower Brushy Canyon sandstones with porosity $>15 \%$ superimposed on 3-D diagram of Bone Spring structure

Fig. 60. Salinity of produced Brushy Canyon waters superimposed on 3-D diagram of Bone Spring structure.

Fig. 61. Salinity of produced lower Brushy Canyon waters and areas productive from lower Brushy Canyon. .85

Fig. 62. Total organic carbon content, in weight percent, of lower Brushy Canyon source rocks.

Fig. 63. Thermal maturity of lower Brushy Canyon source rocks as determined by Rockeval TMAX values and areas productive from lower Brushy Canyon. .86

Fig. 64. API gravity of lower Brushy Canyon oils. .86

Fig. 65. Oil and gas fields productive from Siluro-Devonian carbonates reservoirs in southeast New Mexico.

Fig. 66. Stratigraphic column of lower Paleozoic strata in southeast New Mexico. .87

Fig. 67. Regional west-east cross section across the Delaware Basin in southeastern New Mexico, showing internal stratigraphy of the Siluro-Devonian carbonate section. .88

Fig. 68. Wells penetrating sub-Woodford Devonian strata in southeast New Mexico. .88

Fig. 69. Structure contour map of Siluro-Devonian carbonates.

Fig. 70. Isopach map of Siluro-Devonian strata in southeastern New Mexico. .89

Fig. 71. Woodford subcropmap.

Fig. 72. Pie diagram indicating relative number of wells that each Siluro-Devonian stratal unit (see Fig. 66) produces.

Fig. 73. Rock_eval productivity index (PI) for the Woodford Shale in southeastern New Mexico.

Fig. 74. Total organic carbon (TOC) content of Woodford Shale in southeast New Mexico.

Fig. 75. Actual WTI oil price vs.30-day future price. Time format.

Fig. 76. Actual WTI oil price vs. 30-day future price. Crossplot format....................................92

Fig. 77. Actual WTI oil price vs. Euro/Yen 90-day future price. .93 


\section{Executive Summary and Objectives}

Incomplete or sparse information on types of data such as geologic or formation characteristics introduces a high level of risk for oil exploration and development projects. "Expert" systems developed and used in several disciplines and industries have demonstrated beneficial results. A state-of-the-art exploration "expert" tool, relying on a computerized database and computer maps generated by neural networks, is being developed through the use of "fuzzy" logic, a relatively new mathematical treatment of imprecise or non-explicit parameters and values. Oil prospecting risk can be reduced with the use of a properly developed and validated "Fuzzy Expert Exploration (FEE) Tool."

This FEE Tool can be beneficial in many regions of the U.S. by enabling risk reduction in oil and gas prospecting as well as decreased prospecting and development costs. In the 1998-1999 oil industry environment, many smaller exploration companies lacked the resources of a pool of expert exploration personnel. Downsizing, low oil prices, and scarcity of exploration funds have also affected larger companies, and will, with time, affect the end users of oil industry products in the U.S. as reserves are depleted. The pool of experts is much reduced today. The FEE Tool will benefit a diverse group in the U.S., leading to a more efficient use of scarce funds, and possibly decreasing dependence on foreign oil and lower product prices for consumers.

This fourth of five annual reports contains a summary of progress to date, problems encountered, plans for the next year, and an assessment of the prospects for future progress. The emphasis during the April 2002 through March 2003 period was directed toward Silurian-Devonian geology, development of rules for the fuzzy system, and on-line software. 


\section{Introduction}

In the first four years of the Fee Tool Project, an immense amount of data on the Delaware Basin has been accumulated. Data on geology, structure, production, regional information such as gravity as well as local data, such as well logs. This data, organized and cataloged into several online databases, is available for the Expert System and users as needed and as appropriate in analyzing production potential. A preliminary map of production potential for the basin has been generated and stands ready to be modified by rules defined both by human experts in exploring the Delaware basin, and by statistical rules defined by the database. We have generated a number of new and useful tools and technologies to support these efforts, including online useable interfaces for neural network analysis (PredictOnline), ranking of potential inputs using fuzzy logic (FuzzyOnline), a preliminary Expert System able to make rudimentary drilling decisions, and a web interface for accessing the databases and Expert System software.

In the next year we will provide a finished Expert System that will run remotely from a browser on nearly any computer. The system will be able to aid in development and drilling decisions for both the Brushy Canyon and Devonian plays by providing readily accessible public information. An interactive and customizable questionnaire coupled with relevant analyses will produce an "Expert" opinion of a prospect in a short time to enhance the work of a human explorationist. Though this on-line system will be secure, many users will feel more comfortable if an off-line version of the software is also available. Given time, an effort will be made to produce a stand-alone version. 


\section{Progress and Discussion of Results}

\section{Computational Intelligence}

Regional Data Analysis

A key component to this study is the analysis of the regional data, not only to provide baseline data to correlate with production potential, but also to provide a source of heuristic rules for the expert system. Four major categories of regional data (gravity, aeromagnetic, structure, and thickness were selected, compiled and finalized during the previous year. Regional gravity surveys cover the entire area of the Delaware basin and have been compiled with an accuracy of a few milligals. The survey measurements are on the order of a few thousand feet apart, but sample point locations are highly variable as gravity is measured in easily benchmarked locations, such as along roadways. Gravity measures variations in density and tends to highlight large-scale regional structures in basement materials and if structure has an impact on maturation, migration or trapping of hydrocarbons in the basin useful information can be obtained.

Regional aeromagnetic data, primarily collected via over-flights, with one-mile spacing, re-gridded to 0.296 miles longitude and 0.346 miles latitude, also exists for the region. Aeromagnetic data highlights contrasts in the magnetic susceptibility between rocks and can help indicate basement blocks, large-scale faults, and possible large-scale alluvial deposits.

The structure of the lower Brushy Canyon was picked on 700 wells in the basin covering a geographically large area. Large-scale maps of structure covering the region were constructed with a kriging algorithm using this data. Structure can play more than one role in trapping and migration of hydrocarbons; two potentially helpful attributes 
used in this study are structural highs and flexures that may be caused by subsurface faulting.

Finally, the wells used to compute structure were used to generate an isopach map for the Brushy Canyon in the region. Thickness may indicate areas of greater potential production and also can indicate pinch-outs and other nonstructural features that may form hydrocarbon migration pathways or traps.

A number of attributes were calculated from the four regional data types. These attributes are first and second derivatives along latitude and longitude; dip azimuth and magnitudes; and curvature azimuths and magnitudes. These values were computed to expose finer scale features in the basic data types that might be useful for correlating with a production indicator. A total of 36 maps were generated using the Zmap tool of Landmark Graphics Release 98 plus interpretation package.

Each of these maps was gridded at a scale of 1320-ft (quarter section) because that is the regulatory spacing for wells in the Brushy Canyon in New Mexico. The gridded data was exported and loaded into the project production database. Our current production database is a subset of the ONGARD database (courtesy of the SW PTTC) containing production information on all New Mexico wells. In this database we have also identified Brushy Canyon wells and using grid locations from the Zmap maps we were able to correlate producing wells with grid numbers. The gridding algorithm we selected is based on a 40 ac minimum well spacing and thus consists of points $1320 \mathrm{ft}$ apart. This gridded data set essentially allows regressions to be formed using the production data as control points (training and testing) and the attribute data as variables. Regressions formed in this manner can be used to predict production in all 60,478 40-ac bins in the basin. 
There are two primary considerations in forming regressions. The first involves the quality of the data being predicted with the generated regression model. To address this, we focused initially on overall data quality and tried to filter the data to improve the likelihood of obtaining robust correlations. The second consideration deals with the choice of attributes or variables that will be used in forming the regression model. An optional consideration is the type of models used: linear models (least squares regression) or more complicated non-linear solutions such as polynomial regressions or neural networks.

For the filtering attempt an average of the first 12 producing months' Hydrocarbon Equivalent $(\mathrm{BO}+\mathrm{MCF} / 6)$ calculated at each well was chosen as the data to be modeled. Figure 1 shows a histogram of average hydrocarbon equivalent produced per month in barrels for the 2257 identified Brushy Canyon wells. The trend of the histogram is approximately an exponential decay function. A more ideal data distribution that simplifies modeling is data that follows a Gaussian distribution. The production data was conditioned with a $\log 10$ filter; Fig. 2 shows a histogram of the production indicator after $\log 10$ conversion. The bulk of the data now follows a roughly Gaussian distribution with some notable outliers on the low end. It may be desirable to remove outliers from the training data if those data are not significant to the solution. In this case, a cutoff of 50 barrels of oil per month was applied to remove the outliers. The data can then be unfiltered once the outliers are removed and used as normal in regression analysis as the filtered data is well conditioned for either linear or non-linear regression analyses. However, the 2257-well data set was found to be unwieldy and time-consuming to run regression experiments with and instead we used a smaller subset of the wells that had been examined closely, but not filtered, for geologic data as a training data set. 
For the second consideration, choice of attributes, there are a number of ways to determine which of a set of inputs (attributes) would best be used to form a regression for a particular output. Simply crossplotting each input against the output can give an indication of the quality of potential linear models, but more complicated schemes are required for nonlinear problems. The next subtopic deals with the software developed to address this concern.

\section{Fuzzy Ranking Revisited}

A fuzzy curve solution to the problem of identifying important neural network input variables from a large sparse database was discussed in the second annual report. This first attempt at fuzzy ranking required a certain expertise to use effectively; for this project we prefer to use as much automated software as possible, to avoid variances between interpretive styles. For completeness this "first stage" fuzzy ranking concept is reviewed. In a later section this initial fuzzy ranking concept is expanded and developed into useable, fully automated software.

First stage fuzzy ranking. There are a number of ways to select the best set of inputs to be used to form a regression for a particular output. Simply crossplotting each input against the output can give an indication of the quality of linear or multiple linear regression models that could be formed. Software was developed based on a single stage fuzzy-ranking algorithm to select inputs best suited for predicting the desired output. The global algorithm statistically determines how well a particular input could resolve a particular output with respect to any number of other inputs using fuzzy curve analysis.

To illustrate the technique a simple example is given. Consider a set of random numbers in the range $\{0,1\}$ using $x=\left\{x_{i}\right\}, i=1,2, \ldots, 99$, and $x_{i}=0.01 * i$, and plot each value 
$\left(\mathrm{y}_{\mathrm{l}}=\operatorname{Random}\left(\mathrm{x}_{\mathrm{i}}\right)\right)$. Next add a simple trend to the random data $\left(\mathrm{y}_{\mathrm{i}}=\left(\mathrm{x}_{\mathrm{i}}\right)^{\wedge} 0.5+\operatorname{Random}\left(\mathrm{x}_{\mathrm{i}}\right)\right)$ and plot those values. For each data $\left(\mathrm{x}_{\mathrm{i}}, \mathrm{y}_{\mathrm{i}}\right)$ a "fuzzy" membership function, $\boldsymbol{F i}(\boldsymbol{x})$, is defined using the following relationship:

$$
F_{i}(x)=\exp \left(-\left(\frac{\left.x_{i}-x\right)}{b}\right)^{2}\right) * y_{i}
$$

Sample fuzzy membership functions are shown in Figs. 3 and 4. Here, $b=0.1$, since $\mathrm{b}$ is typically taken as about $10 \%$ of the length of the input interval of $\mathrm{x}_{\mathrm{i}}$. A fuzzy curve was constructed using a summation of all individual fuzzy membership functions in $\left(\mathrm{x}_{\mathrm{i}}, \mathrm{y}_{\mathrm{i}}\right)$, and this final curve can prioritize a set of inputs for linear or non-linear regressions. The fuzzy curve function $\mathbf{F C}(\mathbf{x})$ is defined below:

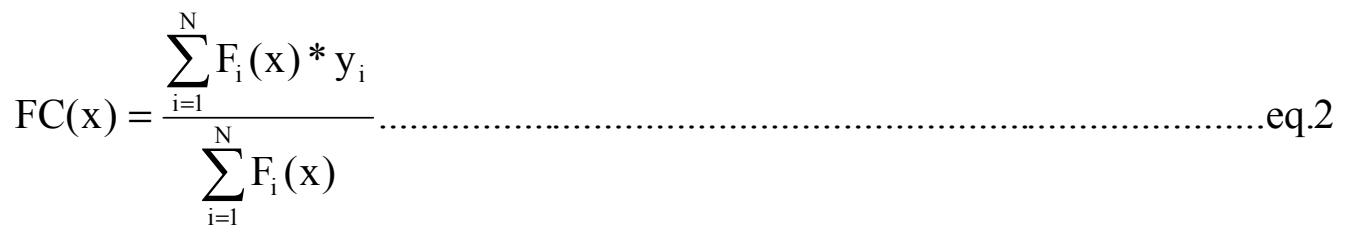

where $\mathrm{N}$ is the size of the data set or the total number of fuzzy membership functions. Figure 5 shows the curves for the data sets shown in Figs. 3 and 4. This simple example illustrates the ability of the fuzzy ranking approach to screen apparently random data for obscure trends such as the correlation between seismic attributes and reservoir properties (Balch et al., 1999).

Second stage fuzzy ranking. More information is needed to advance this analysis from the art of reading these fuzzy curves to a more robust and systematic elimination of less useful inputs. Therefore, work began on a two-stage fuzzy ranking code (Lin et al., 1998). The two-stage fuzzy ranking (TSFR) has two improvements: 1) Reduction of input variable space through random characterization and 2) Setting hard rules for selection of best-input variables. TSFR introduces second stage fuzzy curves, with first and second stage fuzzy surfaces that select the most important and independent input 
variables for modeling, while removing the input variables that show random characteristics.

TSFR uses first and second stage fuzzy curves to generate the fuzzy curve performance index $\left(\mathrm{P}_{\mathrm{c}}\right)$ :

$$
P_{c}=\frac{P_{\text {stage } 1}}{1+P_{\text {stage } 2}}
$$

With the addition of a known random variable into the input space the $\mathrm{P}_{c}$ is normalized by the random $\mathrm{P}_{\mathrm{c}, \mathrm{R}}$ to produce the normalized fuzzy curve performance index $\left(\mathrm{P}_{\mathrm{c}, \mathrm{N}}\right)$ :

$$
P_{c, N}=\frac{P_{c}}{P_{c, R}}
$$

The input variable with the smallest $\mathrm{P}_{\mathrm{c}, \mathrm{N}}$ value is the most important variable. Input variables with $\mathrm{P}_{\mathrm{c}, \mathrm{N}}$ greater than one are eliminated from the selection process. Once the most important variable is determined fuzzy surface analysis is performed.

Analogously, for fuzzy surfaces there exists a performance index using the first and second stage fuzzy surfaces $\left(\mathrm{P}_{\mathrm{s}}\right)$ :

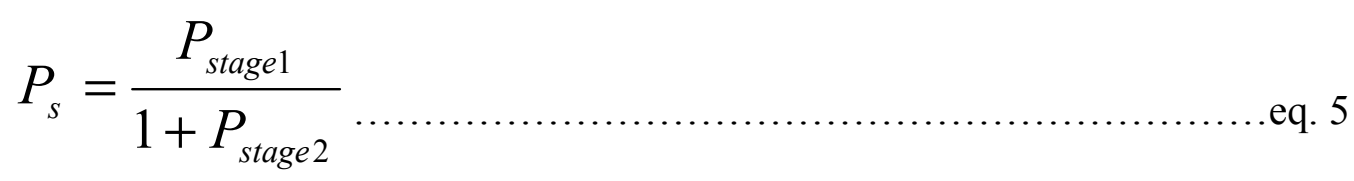

A similar normalization procedure produces the normalized fuzzy surface performance index $\left(\mathrm{P}_{\mathrm{s}, \mathrm{N}}\right)$ :

$$
P_{s, N}=\frac{P_{s}}{P_{s, R}}
$$


Iteratively, the input variable with the smallest $\mathbf{P}_{\mathbf{s , N}}$ is considered the next most important and independent input. In an iterative process, the input variables with $\mathbf{P}_{\mathbf{s}, \mathbf{N}}$ 's above 1.0 are eliminated from selection process. The fuzzy surface analysis continues until no input variables remain. In this way, two-stage fuzzy ranking can be used to automatically and quickly identify the important, independent inputs needed to model the system of interest.

Case study. For this study each of the 36 data and data attributes calculated and loaded into the database were analyzed using fuzzy ranking (Lin and Cunningham, 1995). It is both statistically dangerous and not computationally feasible to use all 36 attributes to form a regression relationship, so two-stage fuzzy ranking software was used to select attributes best suited for predicting production indicators. The algorithm statistically determines how well a particular input (regional data or data attribute), using fuzzy curve analysis, could resolve a particular output (production indicator) with respect to any number of other inputs. Using the newly refined fuzzy ranking tool each data attribute was ranked for its ability to predict the production potential at these well locations. Figure 6 shows the progressive elimination of non-unique and irrelevant data. The four best attributes selected were dip azimuth of gravity, second latitude derivative of thickness, longitude derivative of gravity, and longitude derivative of structure, as shown in Table I.

Table 1. Variables Selected as Optimal

\begin{tabular}{||l|l|l||}
\hline RANKING & VARIABLE & $P C / P C R$ \\
\hline 1 & 6 & 0.8584718 \\
\hline 2 & 32 & 0.8490006 \\
\hline 3 & 2 & 0.8779765 \\
\hline 4 & 20 & 0.88185640 \\
\hline
\end{tabular}


Currently an on-line implementation of this software has been developed and is operating in-house while undergoing testing prior to making it globally available via the web.

\section{PredictOnline Webware Revisited}

For in-house neural network analysis, a web-based software, PredictOnline, was developed. More precisely, it is a web-based interface to SNNS-Stuttgart Neural Network Simulator developed at the University of Stuttgart, Germany and the University

of Tübingen, Germany. [*] SNNS is a comprehensive neural network package featuring a full GUI. It can also be used without its GUI, using a special SNNS program called batchman. This program is the engine of PredictOnline.

Since PredictOnline is "web-based" it means that end users can run the program through their web browsers on local computers connected to the Internet. The computation will be performed remotely on our server machines. Thus, the users are not limited by the speed of their local computers, as neural network training can be computationally demanding.

\section{Multivariate Regression}

Using PredictOnline, a regression relationship was formed between the four fuzzy ranked inputs (Table 1) and the average first month's production for the first year at each of 520 carefully examined Delaware wells. When forming regressions, it is best to hold out a randomly selected sample of the data for testing. This data is used for testing the ability of the regression to accurately predict data not used in forming the regression. For this study a 520 well subset of the available 2434 Brushy Canyon wells in the basin was 
used to train the neural network. These 520 wells were selected because they were verified to have produced only from lower Brushy Canyon, and because they included dry holes in which a completion effort was made to generate production. In addition, they were distributed fairly evenly across the basin. Of these 520 wells, 466 were used to form the regression while 54 were held out for blind testing. A 4-10-10-10-1 neural network with 250 weights provided an excellent solution with a 2:1 ratio of data to weights, $\mathrm{CC}=0.90$ for the training data, and $\mathrm{CC}=0.81$ for the test data. For simplicity, BOPM (average barrels of oil per month expected in first year) at all 60478 40ac-bins in the basin were predicted using this model, including nearly 2000 other wells with Brushy Canyon production. Training and testing cross-plots are shown in Figs. 7 and 8, respectively.

\section{Results}

The calculated BOPM for each 40-ac bin in the New Mexico portion of the Delaware basin, Brushy Canyon formation was used to generate a map (Fig. 9) to highlight potential areas of exploration interest. Neural network analysis necessarily results in a non-crisp solution; examination of the cross-plots in Figs. 7 and 8 demonstrate that there is some possible error in the maps, though in general the high cross-correlation means the overall fit is good. Therefore it would be inappropriate to expect that any given drilling locations would produce exactly as mapped, as there are simply too many variables and the algorithm is designed to form generalized solutions, as seen in Fig. 10. The objective of this project is to use this potential oil rate map as the basis for an expert system that will then quantify the risk associated with each prospect by answering questions often posed by human experts exploring in the Brushy Canyon, 
as well as questions posed by statistical analyses of the data itself. The Map and associated database also provides a rich source of valuable heuristic rules for the expert system.

\section{Expert System Rules}

Basic design changes. The original design entailed the use of a single massive expert system to make decisions about a prospect's potential as a well site, Fig. 11. As we have investigated the process of designing and running expert systems, it has become apparent that a multi-tiered system, with components running in parallel, would be both more efficient and more versatile in actual usage. Figure 12 shows the current design structure for implementing and accessing the various expert systems needed to evaluate production potential. The new design is more efficient for several reasons. First, it will be faster to code the rules and the resulting code will run faster. Second, parallel expert systems will allow the user to consider only the data types they feel are most influential, and ease customization to their personal philosophies. Third, database entry, IO, from the system, will occur in numerous small packets instead of large chunks and extraneous data transfers will be reduced.

Implementation. Figure 12 shows the basic layout of the FEE Tool project. Tier 1 is a user interface that allows selection of an area or prospect of interest. Users can select the types of data they are interested in, and can review that data online with their browsers. Tier 2 in Fig. 12 represents the access of the user's browser with our online database. Advanced users can manipulate the transferred data for personal use. This data will reside on the user's computer and will not be generally available or affect the permanent database in any way. This allows the use of proprietary information with the system. Once the data is accepted or modified, the next step is to run the appropriate 
expert systems using the available data to answer heuristic questions and accepting user input to answer other questions that "experts" tend to ask when evaluating Brushy Canyon prospects. In Tier 3, there are five expert systems that can be applied based on user wishes. These address Regional Indications, Trap Assessment, Formation Assessment, Improved Recovery, and Oil Price. Specifics and starting rules for these five systems are discussed below. Some users may elect to not factor in certain aspects, or to hard wire their own values for future oil price.

Types of rules. Two main types of rules are implemented. Heuristic rules are derived directly from our analysis of regional and local data. These rules are interpreted from the data using algorithms, such as distance relationships, and will be based on publicly available data. Heuristic rules include elements like proximity of mature source rocks, structural pinchouts, nearest producing well, and formation thickness. Expert rules will come from interviews with Delaware explorationists and will mimic questions they ask when evaluating prospects. Expert rules may include information about position on structure, porosity or permeability ranges, and production at analogous sites. In addition, heuristic rules can be replaced if the user has more detailed knowledge than is publicly available. Both types of rules may be fuzzy, as appropriate. Generally, for sites with less information heuristic rules will be more important and will provide a first estimate of production potential. For sites with sufficient specific or proprietary information Expert rules will be dominant.

Heuristic rules. One source of rules for the Fuzzy Expert Exploration Tool is statistical analyses of gridded data in our databases. Currently the regional database has four basic data types for the Brushy Canyon: Gravity, Aeromagnetic, Structure, and Thickness. An additional eight attributes for each of those four basic types has been 
calculated: DX, DY, DX2, DY2, dip azimuth, dip magnitude, curvature azimuth, and curvature magnitude. Additional data include location information in latitude/longitude, oilfield $\mathrm{X}-\mathrm{Y}$ coordinate systems, and a numeric grid number that also functions as a database key. Additionally, in grids that contain a Brushy Canyon well, there is relevant production information for oil, water, and gas. One factor that complicates working with the databases is the fact that the grid is not square: rather, it runs linearly from north to south increasing by integer amounts from the top of the study area to the bottom. The grid then steps over to the next "column." Each gridpoint is separated by a physical distance of $1320 \mathrm{ft}$ that corresponds to an area of 40 acres contained by four adjacent (squared) gridpoints. The gridding system looks something like this:

081318

0409141923

01051015202427

02061116212528

03071217222629

The primary use of the regional database thus far has been to organize the regional data, determine which bins contain production information, and then to calculate a "first guess" map of production potential using the data with the highest fuzzy rank to predict production. With this "first guess" map we enter a new phase, where we want to modify the probability of a successful well prediction using the "first guess" as a baseline. To do this we are using an expert system programmed in Jess and designed to ask the same sort of questions a human explorationist would, when looking at a prospect. Is it high on structure? Where is it in the basin? Are there stratigraphic traps; is it close to other producing wells? A lot of these questions can be answered directly from the 
database itself, which speeds the process of examining a prospect. As a bonus, gridded heuristic answers (e.g. closeness to nearest producing well) also provide us with a way to quantify the value of these answers using fuzzy ranking.

Simple heuristic rules. The simplest questions to ask of the database are related to distances. How close is the nearest producing well? How close to structural highs or lows? Where on structure is the location? Questions about the thickness and existence of potential pay zones at the location of interest can also be answered in this manner. The following new data is being generated for the database:

- Distance to nearest oil show

- Distance to nearest production

- Distance to nearest production $>500$

- Distance to nearest production $>1000$

- Distance to nearest production $>1500$

- Distance to nearest production $>2000$ etc

- Nearness to structural high/low

- Where is the prospect on structure? Scale between local minima and maxima (0100)

- Distance to nearest pinchout (structural trap) defined by sand thickness.

- Direction of nearest pinchout (updip downdip, across trend)

- Relative sandstone thickness (\% distance between local maxima and minima)

- Sandstone thickness relative to nearest producing well (greater or lessser)

- What part of the basin does the prospect lie in? (defined as distance from key points?)

-West edge 
-Center

-North edge

-East edge

Scoring of rules. Each of the subexpert systems will assign a numerical score based on the answers to individual questions. The score can come in several varieties: binary, or off/on flagging, assigned percentage values, or fuzzy-based distributions. Most rules in the subsystems will likely be assigned numerical values based on analysis of training data or fuzzy distributions based on data analysis. When combined to form the global relationship, fuzzy distributions or other functions will be applied.

Grading heuristic rules. One function of the new fuzzy ranking software is that it numerically grades, and sorts by uniqueness. We can now take suites of data, compare them to some desired quantity, and rapidly determine which are better (numerically ranked higher), and which add no new information (uniqueness test). Suites of heuristic rules can be compared to production and a numeric grade assigned based on the fuzzy rank and level of uniqueness.

Trap assessment rules. Initial trap assessment rules as programmed into the initial system are graphically illustrated in Figs. 13-15 in flowchart form.

Formation assessment rules. Initial source rock assessment rules as programmed into the initial system are graphically illustrated in Figs. 16-20 in flowchart form.

Regional indication rules. Initial regional assessment rules as programmed into the initial system are graphically illustrated in Figs. 21-22 in flowchart form.

Improved recovery rules. As the Brushy Canyon sands are a relatively new play, little information is available on improving existing production. Also, as the play is 
believed by some to be water-wet, waterfloods are high risk anywhere in the basin. Flooding with $\mathrm{CO}_{2}$ is a possibility for advanced recovery techniques, but Brushy Canyon $\mathrm{CO}_{2}$ field application data is scarce. Horizontal wells appear to be the most likely candidate technology for improved recovery.

Oil price rules. Oil price will be available in three main formats.

- $\quad$ User entered: the user enters a fixed price per barrel based on internal projections or company philosophy.

- $\quad$ Predicted by neural network: a neural network is being developed to project the price of oil using the futures markets. The status of the oil price predictive method is discussed later in the report.

- $\quad$ Standard posted price for the day: the calculation is made is the third option.

Enhancements and reductions of rule values. There are many methods available to enhance or reduce the estimates. The method used at this stage of development is to enhance an estimate by taking roots as all values are normalized between Zero and One. The cube root is used to strongly enhance the value, and the square root is used to moderately enhance the value. To reduce the estimate, it is raised to the second power and to strongly reduce, it is raised to the third power. In the case of the thickness modification, a variation is used in which the enhancements are smaller, created by taking the 1.25 root and the 1.5 root instead of the square and cube root.

Statistical analysis of rules. Statistical analyses of the data are useful in determining data-based estimates of ranges to be used. For example, in order to 
determine cutoff values for determining if values such as dip or thickness are significant enough to result in an enhancement or reduction of the estimate, a histogram of the variable in question can be drawn, and tests for normality performed. If the distribution of the variable seems close to normal, cutoff values can then be determined by taking the mean, plus or minus a standard deviation, for a moderate enhancement and reduction, and the mean, plus or minus two standard deviations. for a strong enhancement.

For example, when we observed thickness data, the data appeared to be skewed to the right (Figure 23). This caused the majority of the variables to be enhanced, as can be seen by looking at the distribution of the differences in the initial value and the final value after the first branch enhancements are completed (Figure 24).

The difference between the initial value and final value is one tool to evaluate how the expert system is performing. In the first trial, all enhancements and reductions were accomplished by using the square and cube roots and the second and third powers respectively. When the study of the differences between initial value and final value showed that the majority of final estimates were higher than the corresponding initial estimate for every grid-point, the thickness enhancement was found to be the cause. The goal is to have a symmetric distribution when looking at the differences. In other words, if prospect potential is indeed distributed approximately normally, the mean, median and mode difference should be zero. To achieve this distribution of the differences, a new method of enhancement based on the thickness was used as discussed above.

This is an example of how statistical analysis of the raw data itself may aid in forming a robust system, when combined with Expert-derived rules. 
Numerical Results from Non-Numerical Rules.

Introduction. Fuzzy set theory is a mathematical approach for working with imprecise data and measurements. In exploration, relevant data such as porosity is sometimes approximated or interpolated from data collected at nearby wells. This example shows how principles of fuzzy set theory are used along with expert opinions to compute a value for a well's potential. The steps involved are: determining the input parameters and obtaining approximate numerical values, developing the linguistic values, fuzzifying the input parameters, firing the appropriate expert defined rules, and defuzzification of the output parameter. Each of these steps is discussed in detail below.

Input parameters. In this example, two variables will be used as input parameters. The variables, total organic carbon $(\mathrm{T})$ and porosity $(\Phi)$ are variables for which it is sometimes difficult to get a precise value, and measurements may have to be used from nearby wells. For each of these variables, linguistic values will be defined based on the following criteria:

$T=$ Total Organic Carbon

$\mathrm{T}$ : ZERO if $0 \leq \mathrm{T}<0.5$

$\mathrm{T}$ : LOW if $0.5 \leq \mathrm{T}<1.0$

T: MEDIUM if $1.0 \leq \mathrm{T}<1.5$

T: HIGH if $1.5 \leq \mathrm{T}$

$\Phi=$ Porosity (percentage)

$\Phi$ : ZERO if $0 \leq \mathrm{P}<5$

$\Phi$ : LOW if $5 \leq \mathrm{P}<10$

$\Phi$ : MEDIUM if $10 \leq \mathrm{P}<15$

Ф: HIGH if $15 \leq \mathrm{P}$

For this example, 0.72 will be used as the best available value for TOC, and $13 \%$ will be used for the best available porosity. These two inputs will be used to develop a value for $\mathrm{R}$, the prospect potential on a scale of 1 to 100 . 
Fuzzification of input parameters. The next step in the process is to "fuzzify" the input parameters. In order to do this, we will define fuzzy membership values for each of the sets; zero, low, medium and high, using a set diagram called a fuzzy membership curve that graphically defines each of the linguistic values. There are many curves that can be used in this process (and a suite was tested and reported later in this report) but the simplest is a trapezoidal graph, which we will use here for purposes of illustration. The process is repeated for each of the input parameters. Figure 25 illustrates the process for the variable $T$. The value of 0.72 is plotted on the $\mathrm{x}$-axis, corresponding to the following values of membership in each of the linguistic sets:

$\mathrm{T}($ Zero $)=0$

$\mathrm{T}(\mathrm{Low})=56$

$\mathrm{T}($ Medium $)=44$

$\mathrm{T}($ High $)=0$

The process is repeated for the porosity (Figure 26), using the best value of $13 \%$.

$\Phi($ Zero $)=0$

$\Phi($ Low $)=0$

$\Phi($ Medium $)=40$

$\Phi($ High $)=60$

Rules. Once the input parameters have been fuzzified, the linguistic sets with nonzero membership can be used to fire a set of rules determined by an expert. The rules for this example are

1. If $\mathrm{T}$ is zero then $\mathrm{R}$ is zero

2. If $\Phi$ is zero then $R$ is zero

3. If $\mathrm{T}$ is low and $\Phi$ is low or medium, then $\mathrm{R}$ is low

4. If $T$ is low and $\Phi$ is high then $R$ is medium

5. If $T$ is medium and $\Phi$ is low then $R$ is low

6. If $\mathrm{T}$ is medium and $\Phi$ is medium or high, then $\mathrm{R}$ is medium

7. If $\mathrm{T}$ is high and $\Phi$ is low or medium then $\mathrm{R}$ is medium

8. If $\mathrm{T}$ is high and $\Phi$ is high then $\mathrm{R}$ is high 
We use the non-zero memberships from the fuzzification process to determine that rules 3, 4 and 6 are applicable.

Defuzzification. The next step in the process is to determine the strength of each of the fired rules using the set theory operators min for "and" and max for "or". Beginning with rule 3, we have $\mathrm{T}$ low with membership value of 56 , $\Phi$ low with membership value of 0 and $\Phi$ medium with membership value of 40 . So, $\Phi$ is low $\boldsymbol{o r}$ medium with a membership value of 40 . Rule 3 is then "fired" with a strength of 40 , using $\min (56,40)$ to arrive at this value.

Following this process for the two other rules, rule 4 and 6, we have rule 4 fired with a strength of 56 and rule 6 fired with a strength of 44 . Rule 4 and 6 , however, both result in $\mathrm{R}$ being medium, so we combine the two using the max operator. In the final results, $\mathrm{R}$ is medium with strength of 56 and low with strength of 40 .

To obtain a numerical value for $\mathrm{R}$, on a scale of 1 to 100 , we consider the median values of 10 for low, 50 for medium and 90 for high. Then using the strengths computed above, we calculate $\mathrm{R}$ as follows:

$\mathrm{R}=0.40 *(10)+0.56 *(50)=32$

This is a simple example of how the fuzzy set theory approach can be used to determine potential. In a more complex example, multiple input parameters may be used, and the curves used to determine the memberships may be more complex than the trapezoidal curves used here. The basic ideas are the same, however, and can be used to build the framework for computer codes that compute potential based on rules written by experts in the field. 


\section{Initial application of the Expert System}

The Expert System as a whole can be divided into four subsystems (Figure 12). Each of these systems asks questions that an Expert would ask about that sub-topic: Regional Indication, Trap Assessment, Formation Assessment, and Enhanced Recovery. Each subprocess results in numerical values for risk for that component. However, an overall evaluation is also required. During the overall evaluation the user's value for oil price, or a projection, is also factored in using a weighted sum of the risk components.

Risk $=[r(R)(W)+r(T)(X)+r(F)(Y)+r(E)(Z)] * r(O)$

where $\mathrm{W}+\mathrm{X}+\mathrm{Y}+\mathrm{Z}=100 \%$, and where $\mathrm{r}(\mathrm{O})$ is a scaling factor based on Oil Price.

Some thought has gone into the evaluation of these final risk assessment values $\{\mathrm{W}, \mathrm{X}, \mathrm{Y}, \mathrm{Z}\}$, which are critical in weighting each factor before generating a final risk estimate; in these, expert opinion will weigh heavily. Each of the subsystems is also being evaluated for the best method of selecting and defining membership functions for key components. This is the part of the expert system that allows the use of imprecise or fuzzy data, such as "low porosity" or "moderate thickness". Several schemes for determining the best forms to use for these membership functions have been studied. This initial assessment is based in part on statistics of the data and in part on expert opinion. The thickness of pay sand ranges are shown below by way of illustration:

- Zero (sub-marginal, or no pay sands)

- Low (Pay sand thickness is defined as marginal by experts)

- Medium (This range tends to provide successful wells) 
- High (Better than average sand thickness)

All data analyzed within the expert system are normalized to simplify computation; additionally, to start, ranges were selected using the assumption that the distribution of thicknesses was normal.

- Zero $\mathrm{T}<0.2$

- $\quad 0.2 \leq \mathrm{T}<0.5$

- $\quad$ Medium $\quad 0.5 \leq \mathrm{T}<0.8$

- High $\quad \mathrm{T} \geq 0.8$

Matlab provides a number of membership functions, so several were selected to test the process. The first function that was considered for the membership curves is the "trapmf" function. In order to have the memberships functions allow for membership in more than one range (partly high, partly medium for example), a certain amount of overlap on the ranges is desired. Thus the ranges were redefined as follows:

- Zero $\mathrm{T}<0.225$

- $\quad 0.175 \leq \mathrm{T}<0.525$

- $\quad$ Medium $0.475 \leq \mathrm{T}<0.825$

- High $\quad \mathrm{T} \geq 0.775$

Using these ranges the membership curves shown in Figure 27 were generated using a composite set of Trap and Formation (source) rules coupled with Regional Indications 
to predict risk. With these functions prepared, it is then possible to compute the values for rules, and values for a number of circumstances to test the performance of various membership functions.

The framing of rules was done based on the weights of the inputs. In this particular case since the first two inputs were combined as one input, more weight was given to that particular input. Finally, for this highly simplified evaluation, the ranges for rules are defined in Table 2, below, and graphically illustrated in Figure 28:

Table 2. Ranges for Rules

$\begin{array}{lll}\text { Trap and Source } & \text { Regional (NN) } & \text { Risk (output) } \\ \text { Zero } & \text { Zero } & \text { Zero } \\ \text { Zero } & \text { Low } & \text { Zero } \\ \text { Zero } & \text { Medium } & \text { Low } \\ \text { Zero } & \text { High } & \text { Low } \\ \text { Low } & \text { Zero } & \text { Low } \\ \text { Low } & \text { Low } & \text { Low } \\ \text { Low } & \text { Medium } & \text { Low } \\ \text { Low } & \text { High } & \text { Medium } \\ \text { Medium } & \text { Zero } & \text { Low } \\ \text { Medium } & \text { Low } & \text { Medium } \\ \text { Medium } & \text { Medium } & \text { Medium } \\ \text { Medium } & \text { High } & \text { Medium } \\ \text { High } & \text { Zero } & \text { Medium } \\ \text { High } & \text { Low } & \text { Medium } \\ \text { High } & \text { Medium } & \text { High } \\ \text { High } & \text { High } & \text { High }\end{array}$

The numerical, or defuzzified values computed using this set of curves is shown in Table 3 , below. 
Table 3. Numerical Values Generated Using Trap and Source Rules

\begin{tabular}{|l|l|l|}
\hline Trap \& Source & Region & Risk \\
\hline 0.5 & 0.5 & 0.5 \\
\hline 0.142 & 0.5 & 0.254 \\
\hline 0.757 & 0.5 & 0.65 \\
\hline 0.95 & 0.5 & 0.746 \\
\hline 0.95 & 0.932 & 0.902 \\
\hline 0.95 & 0.777 & 0.901 \\
\hline 0.95 & 0.332 & 0.65 \\
\hline 0.445 & 0.332 & 0.35 \\
\hline 0.445 & 0.541 & 0.35 \\
\hline 0.445 & 0.632 & 0.35 \\
\hline
\end{tabular}

We can see in the last three rows of Table 3 that, though the regional input is being varied, keeping trap and source input constant results in no change in the output. This is undesirable and rules out the membership curves in Figure 27.

The next set of functions examined were the "gaussmf" functions shown in Figure 29, determined using the same parameters as the first trial. Figure 30 shows the membership curves using the "gauss $2 \mathrm{mf}$ " function for the zero and high parameters in both the inputs as well as the output. Finally, the "Zmf " function was used for the Zero parameter and the "smf" function for the high parameter in both the inputs as well as the output, with membership curves shown in Figure 31. Table 4 summarizes the results of this trial. 


\section{Table 4. Results of “gaussmf” Function Trial}

\begin{tabular}{|l|l|l|l|l|}
\hline TRAP & REGION & gussmf & gauss2mf & zmf, smf \\
\hline 0.5 & 0.5 & 0.545 & 0.545 & 0.545 \\
\hline 0.28 & 0.5 & 0.331 & 0.335 & 0.32 \\
\hline 0.913 & 0.5 & 0.775 & 0.772 & 0.775 \\
\hline 0.913 & 0.677 & 0.923 & 0.936 & 0.896 \\
\hline 0.913 & 0.814 & 0.884 & 0.899 & 0.887 \\
\hline 0.913 & 0.895 & 0.918 & 0.93 & 0.898 \\
\hline 0.913 & 0.968 & 0.923 & 0.936 & 0.898 \\
\hline 0.913 & 0.123 & 0.647 & 0.647 & 0.648 \\
\hline 0.0413 & 0.123 & 0.087 & 0.0745 & 0.102 \\
\hline 0.124 & 0.123 & 0.101 & 0.0966 & 0.107 \\
\hline
\end{tabular}

Analyzing Table 4 shows that in the case of the fourth and fifth inputs, the trap input remains unchanged; while only the region input increases, the output decreases. As we change the membership functions, this trend continues. This shows the significance of the membership curves, in that they can be used to fine-tune responses to various rules.

The next step was to apply the methodology to some actual project data. Figure 23 shows a histogram distribution of thickness data, demonstrating an approximately normal distribution (though skewed somewhat). In this trial the idea was to build a succession of curves that accurately reflected the value of thickness as the membership functions moved from zero to high.

In order to implement an overall function that demonstrated the characteristics of the actual curve, the "dsinmf" and "psinmf" functions in Matlab were used for both low and medium and the "zmf" and "smf" functions for the zero and high portions of the distribution. Figure 32 shows the fuzzy membership functions. 
For comparison, the similar "pimf" function was applied to the low and medium parameters and the "zmf" and "smf" functions for the zero and high parameters in a separate test case (Figure 33).

Table 5 shows the numerical results from these two sets of fuzzy membership functions. A new problem developed, as inputs 2 and 3 of the region input were being varied while the trap input was held constant. There was no difference in the output for the range of 0.555 to 0.8 in case of the region ional input. Another observation was that decreasing the trap input values while leaving the region ional input values constant resulted in an increasing when the "psinmf" and "dsinmf" functions were used. Of these two tests, the "pimf" function appears to provide the most statistically sound results.

Table 5. Numerical Results from Psinmf/dsinmf, pimf Functions

\begin{tabular}{|l|l|l|l|}
\hline TRAP & REGION & Psinmf / dsinmf & pimf \\
\hline 0.555 & 0.5 & 0.575 & 0.591 \\
\hline 0.5 & 0.555 & 0.524 & 0.591 \\
\hline 0.5 & 0.8 & 0.524 & 0.601 \\
\hline $\mathbf{0 . 5}$ & $\mathbf{0 . 8 5}$ & $\mathbf{0 . 5 7}$ & 0.593 \\
\hline $\mathbf{0 . 4 5 4}$ & $\mathbf{0 . 8 5}$ & $\mathbf{0 . 5 8 3}$ & 0.613 \\
\hline $\mathbf{0 . 4 2 7}$ & $\mathbf{0 . 8 5}$ & $\mathbf{0 . 5 8 5}$ & 0.607 \\
\hline $\mathbf{0 . 2 4 3}$ & $\mathbf{0 . 8 5}$ & $\mathbf{0 . 5 8 7}$ & 0.54 \\
\hline 0.95 & 0.95 & 0.878 & 0.898 \\
\hline 0.821 & 0.95 & 0.773 & 0.89 \\
\hline 0.821 & 0.0955 & 0.556 & 0.597 \\
\hline
\end{tabular}

Based on expert knowledge, however, it was already known what the general form of the output should be, and while these cases were closer, they were not an accurate fit in certain ranges. Further refinements were made using "sin" curves for the Low and Medium membership curves while leaving the zero and high parameter curves defined by "zmf" and "smf" curves. Further refinements were made by shifting the range 
of the High parameter to 0.75 from 0.70 , and by changing adjusting the angle of the sin curve to 50 , then 30 . Figure 34 shows the resulting suite of membership curves.

Table 6. Results from sin Functions

\begin{tabular}{|l|l|l|l|l|l|}
\hline TRAP & REGION & sinmf & sinmf & sinmf & sinmf \\
\hline 0.5 & 0.5 & 0.668 & 0.668 & 0.676 & 0.674 \\
\hline 0.28 & 0.5 & 0.605 & 0.605 & 0.535 & 0.598 \\
\hline 0.913 & 0.5 & 0.668 & 0.668 & 0.676 & 0.674 \\
\hline 0.913 & 0.677 & 0.671 & 0.671 & 0.676 & 0.675 \\
\hline 0.913 & 0.814 & 0.671 & 0.671 & 0.676 & 0.675 \\
\hline 0.913 & 0.895 & 0.671 & 0.671 & 0.676 & 0.675 \\
\hline 0.913 & 0.968 & 0.671 & 0.671 & 0.676 & 0.675 \\
\hline 0.913 & 0.123 & 0.669 & 0.669 & 0.675 & 0.673 \\
\hline 0.0413 & 0.123 & 0.106 & 0.106 & 0.102 & 0.102 \\
\hline 0.124 & 0.123 & 0.122 & 0.122 & 0.102 & 0.104 \\
\hline
\end{tabular}

When the regional parameter is changed without varying the trap for a range of 0.677 to 0.968 , the results do not change at all. Several more tests were conducted, varying the values of the various ranges (Zero, Low, Medium, and High) to further explore available functions and ranges, but ultimately a sample of real prospect evaluations will have to be computed and then statistically examined to recognize the final shape the memberships curves require.

\section{WEB-Based Database Management System (WDMS)}

A key component to the success of this project is the development of a dynamic web-accessible database for storing, managing, accessing, and analyzing data, including the development of heuristic fuzzy rules and operating the inference engine. As the data files can be quite large, the system must be efficient, and usable by persons with varying degrees of computer literacy. 
Several important advances in the web system have taken place in this semiannual period. Key data definitions, data flows, data processing methods and user interfaces with WDMS have all progressed. A new version of PredictOnline has been coded, user management software has been developed and debugged, and a beta version of a two-stage fuzzy ranking code, FuzzyOnline, utilizing the more advanced software, has been developed.

Considerations of security for both users and potentially proprietary data, as well as for the integrity of the databases, led to the development of Web-Based Account Management. Users can now register, login, create and delete accounts, change user's privilege and see statistics on their personal usage of the system in a secure environment. In addition, system administrators can locally or remotely manage all accounts.

Improvements to PredictOnline include the removal of the java policy file. In the previous version, a java policy file was needed on the user's computer to allow PredictOnline to access data via applets. Upload and download functions without java policy files have been developed in JSP. Also, the neural network algorithm that we favor (conjuate gradient algorithm) was compared with a newer variant, which improved efficiency by nearly an order of magnitude for a test case, but did not blind-test very well; thus we are using our current time-tested algorithm in PredictOnline.

Java applets codes were designed to implement the user-side interface for a new FuzzyRank software package. JSP codes were designed to implement the server-side functions. JSP codes, called Fortran executable codes, have been successfully tested, a java version is in beta testing now in-house, and will soon be available for general use by consortium members. 
The development of interactive software allowing users to examine, crossreference, and in the case of better proprietary knowledge, adjust data is a core component of the final Expert System. A java-based software tool to accomplish this has been completed and is being tested now on our existing Rules base.

\section{Engineering}

\section{Lower Brushy Canyon Clastics}

Prior to the advent of a precise sidewall coring procedure, noncommercial Brushy Canyon completions were a problem. A new method based on artificial intelligence to predict bulk volume oil from conventional logs was developed

The methodology was developed with the Nash Draw \#23 log-core information and was detailed in the Second Annual Report. In brief, it is known that operators rely on core analyses of porosity and oil saturation to make their Brushy Canyon completion decisions. Artificial intelligence tools, fuzzy logic and neural networks, were used to select the logs and to develop correlations with core measurements of the product of porosity and oil saturation (bulk volume oil), with the potential of reducing or eliminating the need for core information.

Fuzzy ranking was used to prioritize the logs on a global basis that best correlated with bulk volume oil, BVO, as measured in a $200 \mathrm{ft}$ whole core from the Nash Draw Field. The density and neutron porosity logs, along with the deep and shallow resistivity logs, were then used as inputs to a neural network to develop a multivariate regression equation (trained neural network) that correlated the four logs with BVO. The Nash Draw 23 neural network was found to be robust by blind-testing it with logs and core data 
from 18 additional wells in the Nash Draw Field and 14 wells in the North Lee Field. The blind testing generally fit the BVO patterns evident in the limited core data.

The trained and tested Nash Draw 23 neural network was used to generate pseudo-BVO logs on 34 Lower Brushy Canyon wells in the NM Delaware basin. To reduce the risk of non-commercial completions, the 34 BVO pseudo-logs were compared to the average oil rate of each of the wells' first 12 producing months. A simple neural network architecture (3-4-1) was developed to correlate the statistical parameter's sum, average, and standard deviation of the pseudo-BVO log with the production indicator. Hence, using the sequential neural network approach, given the four logs through the Lower Brushy Canyon, a pseudo-BVO log can be generated and the statistical description of the log can be used to estimate the well's first year monthly production.

In the third year reporting period, the methodology was applied to a new Lower Brushy Canyon dataset (Poker Lake Field) and to a carbonate reservoir dataset. The Poker Lake Field dataset demonstrated the pitfalls that can occur with limited training data.

Two Poker Lake wells had core data for calculating BVO. Well 89 had 19 core measurements (samples) while well 80 had 79 samples. The well 89 dataset nicely illustrates the pitfall of insufficient data. An insufficient number of samples can result in "over-training." Over-training is illustrated by the cartoon in Fig. 35. The fictional 11sample dataset illustrated in Fig. 35 was divided into a training set and a testing set. The polynomial best-fit line trains to a $100 \mathrm{R}^{2}$ using the five data points. The best-fit straight line trains to an $88 \mathrm{R}^{2}$ using same five data points. Using the trained polynomial curve (dashed line) to predict values could result in large errors, including negative values. The straight-line model predicts values with reasonable error throughout the limits of the 
dataset. A real example of this problem occurred with the Poker Lake 89 dataset. The density and neutron porosity logs along with the deep and shallow resistivity logs were used as input to train a neural network with the limited (19 available) BVO measurements. The training was almost perfect, as shown in Fig. 36. The Poker Lake 89 neural network was used to predict the Nash Draw 23 BVO log shown in Fig. 37. Notice that the predicted high BVO values are in areas where the core values (truth) are low. Experience to date suggests that the ratio of samples to neural network weights (node tie lines) should exceed 2 to minimize the over-training problem. The more global correlations resulting from the Nash Draw 23 dataset adequately predicted the core BVO values of Poker Lake 89 even though the fields are about 10 miles apart.

\section{Carbonate}

Data is currently being assimilated on the Devonian carbonate that exists regionally throughout the Delaware basin. During the initial six months of data collection, no whole core information was uncovered. However, a consortium member was interested in applying the log analyses methodology to a carbonate system of algal mounds. The dataset serves as a test of the method in a carbonate reservoir.

Six wells with a full suite of logs (typical logs shown in Fig. 38) and core data throughout a dolomitic carbonate reservoir were made available to the project. The log suite consisted of caliper, gamma ray, photoelectric effect, laterolog deep resistivity, laterolog shallow resistivity, microspherically-focused resistivity, density porosity, and neutron porosity. Fuzzy curves were generated to rank the association between the log and the respective core-measured BVO. The caliper, shallow resistivity, and the density porosity were found to have a strong relationship with core-measured values of BVO. Examples of good and poor fuzzy ranking, defined as the maximum minus the minimum 
values of the fuzzy curve plus the $\mathrm{R}^{2}$ value of a best fit straight-line through the curve, are shown in Figs. 39 and 40.

The caliper, shallow resistivity, and the density porosity logs from the Dagger Draw 12 well were used to develop a 4-8-4-1 neural network that trained to a $90 \%$ correlation coefficient with 200 core-measured values of BVO. The training is illustrated in Fig. 41. The photo-electric factor, shallow resistivity, and the density porosity logs were used to model a BVG $\log$ defined by the core values of $1-\mathrm{S}_{\mathrm{w}}-\mathrm{S}_{\mathrm{o}}$. The goodness of the 4-6-4-1 neural network training (85\% correlation coefficient) is seen in Fig. 42.

The correlations of caliper, shallow resistivity, and density porosity logs with bulk volume water (BVW) ranked highest and were used to develop a 4-6-4-1 neural network to produce a pseudo-BVW log. The goodness of the $85 \%$ correlation coefficient is seen in Fig. 43.

The same three logs were used to train a 4-6-6-1 neural network to produce an aspect ratio pseudo-log. The aspect ratio logs are defined by $\Phi / \mathrm{k}$ as measured in the 200core values. The goodness of the $80 \%$ correlation training is seen in Fig. 44.

There are an additional five wells with logs and cores available to train and test neural network architectures. The BVO neural network architecture and the best training correlation coefficient for each well is shown in Table 2 along with the number of training samples included in the each wells dataset. Notice that the Dagger Draw 12 well with the 4-8-4-1 neural network produced the best training correlation coefficient. The same is true for the BVG and BVW neural networks.

The Dagger Draw 12 BVO and BVG neural networks were used to generate pseudo-BVO and -BVG logs for the five wells with core information. As a blind test the pseudo-logs were compared to the core measurements in Figs. 45 and 46. The statistical 
parameter's sum, average, and standard deviation for each pseudo-log were calculated and cross-plotted with the average of the first producing year's monthly oil and gas production. The cross-plots are shown in Figs. 47 and 48.

Lastly, two of the statistical parameters were used to generate correlations to predict oil, gas, and water production. The dataset is limited to the six wells previously discussed, so a simple neural network architecture (2-1-1) with Sum and Avg as the inputs was used to develop the production correlations shown in Figs. 49 and 50 used to predict oil and gas rates. Water production predictions were poor.

\section{Geology}

\section{Brushy Canyon}

The Brushy Canyon Formation of the Delaware Mountain Group (Permian: Guadalupian) consists of 550 to $1650 \mathrm{ft}$ of interbedded fine-grained sandstones, siltstones, and minor detrital carbonates deposited in the deep-marine Delaware Basin (Fig. 51). The Brushy Canyon Formation unconformably overlies the Bone Spring Formation (Permian: Leonardian) or erosional remnants of the Cutoff Formation (Permian: Guadalupian) throughout much of the Delaware Basin (Fig. 52). The Brushy Canyon is overlain by basinal sandstones and siltstones of the Cherry Canyon Formation (Permian: Guadalupian). In New Mexico, depth to the Brushy Canyon ranges from 1950 $\mathrm{ft}$ near the northern and western margins of the basin to $8600 \mathrm{ft}$ in the deepest part of the basin in southwest Lea County.

The Brushy Canyon became a major exploration and development target in the middle to late 1980s. It had been drilled through and ignored for decades as producers concentrated on deeper targets, mainly gas-productive intervals in the Morrowan and 
Atokan (Lower Pennsylvanian) and also on shallower oil-productive sandstones of the Bell Canyon Formation of the Delaware Mountain Group (Permian: Guadalupian). The Brushy Canyon sandstones were not considered a viable exploration target for four reasons (Montgomery et al., 1999). First, they are low-resistivity sandstones for which conventional log analysis indicates high water saturations. Second, they are less permeable than shallower oil-productive sandstones in the Bell Canyon. Third, the Brushy Canyon is a deeper target than the Bell Canyon, therefore making it seem not as economically viable. Fourth, little or no oil flows from Brushy Canyon sandstones on drill-stem tests. Since the mid-1980's, however, the Brushy Canyon has been recognized as a major, economically viable target. More than 110 fields currently produce oil and associated gas from the Brushy Canyon in southeast New Mexico. The predominant trapping mechanism is stratigraphic (Montgomery et al., 1999).

The Brushy Canyon has been subdivided into three informal stratigraphic units by most workers, and upper unit, a middle unit, and a lower unit (Fig. 52; Montgomery et al., 1999). Boundaries between the units are not well defined and generally vary from worker to worker. The upper Brushy Canyon produces significant volumes of oil and the middle Brushy Canyon produces relatively little oil. The lower unit has produced most of the oil obtained from the Brushy Canyon. This part of the project concentrated on the lower Brushy Canyon.

Brushy Canyon fields have typically been discovered by reexamining and reentering old wells that have produced from deeper stratigraphic units such as the Morrow (Lower Pennsylvanian) but have been abandoned as the deeper production has declined to subeconomic levels. Recompletions are typically made in those wells that exhibited good mudlog shows in the Brushy Canyon but were never tested or were tested 
with water recovery on a drill-stem test. A few operators have drilled new wells updip of older wells that have exhibited shows in the Brushy Canyon. Generally, however, most discoveries have been made by reentering old wells. Most exploration, therefore, has been concentrated on wells that have been drilled to Lower Pennsylvanian targets. While this has proved to be a successful and economically viable strategy for many operators, it has largely limited exploration to areas where recently abandoned deep wells are available for reentry. Because many of the wells were originally drilled on structures that affect the Lower Pennsylvanian, most Brushy Canyon exploration has therefore been essentially structural in aspect and has largely ignored the stratigraphic component of trapping that is dominant.

This portion of the project involved the acquisition, synthesis, and analysis of structural, stratigraphic, production, reservoir, and source-rock data. The data are collected from more than 720 wells basinwide (Fig. 53). Wherever possible, data were collected so that data from productive wells in Brushy Canyon pools were offset by nonproductive wells adjacent to those pools. In this way, geologic contrasts between productive areas and immediately adjacent nonproductive areas are reflected in the dataset and its derivative maps. Data synthesis and analysis performed as this part of the project have been used to help devise and structure the neural network system.

The structure on top of the Bone Spring Formation (Fig. 54) and lower Brushy Canyon Formation (Fig. 55) indicate more than $7500 \mathrm{ft}$ of structural relief between the shallower parts of the basin to the north and the deepest part of the basin to the southeast. The structure on the top of the Brushy Canyon Formation (Fig. 55) is very similar with a relief of only $7200 \mathrm{ft}$, indicating a partial infilling of the basin during Brushy Canyon time. The structure on the top of the Bone Spring Formation includes numerous local 
closures and structural noses that are superimposed on the regional descent of the Bone Spring into the Delaware Basin. Also present are several structural lows that extend into the basin from adjacent areas on the Northwest shelf. As discussed below, many of these local structures controlled sand distribution in the lower Brushy Canyon and are therefore paleobathymetric elements that were in existence at the time of Brushy Canyon deposition. Many, but certainly not all, appear to be located over Pennsylvanian structures described by Montgomery et al. (1999). Pennsylvanian- and Permian-age structure has been overprinted by a southeast regional tilt of Laramide (Late Cretaceous Early Tertiary) age (Hills, 1963; Dickerson, 1985).

The lower part of the Brushy Canyon Formation is 60 to $500 \mathrm{ft}$ thick within the project area in southeast New Mexico (Fig. 56). It consists of arkosic to subarkosic, finegrained sandstones (reservoir facies), organic-rich siltstones (seal and source-rock facies), dolostones and limestones that are present mostly near the shelf margin, and black to dark-gray calcareous shales or marlstones (Montgomery et al., 1999).

Brushy Canyon sandstones are allochthonous sediments that originated on surrounding shelf areas and were transported into the deep-marine Delaware Basin prior to deposition. The mechanism of transport is enigmatic and has been ascribed more recently to various gravity-related flow processes including saline density currents (Harms, 1974; Harms and Williamson, 1988; Harms and Brady, 1996) and turbidity currents (Hull, 1957; Jacka et al., 1968). Whatever the mechanism of transport, the conveying currents apparently flowed downslope and deposited the sands in submarine channels and on submarine fan and channel complexes (e.g. May, 1996; Thomerson and Catalano, 1996; Basham, 1996; Broadhead et al., 1998). Most depositional models hypothesize that Brushy Canyon sands were initially transported across the Northwest 
shelf during lowstands of sea level when the shelf was exposed. The sands were then remobilized and transported into the basin through submarine canyons incised into the shelf-edge carbonates.

Thicker areas of the lower Brushy Canyon were deposited in structurally low depressions in the underlying Bone Spring Formation. Many of these depressions originate at the shelf edge and are elongate perpendicular to the shelf edge. This relationship between lower Brushy Canyon thickness patterns and underlying Bone Spring morphology indicates that the structural depressions were depressions that acted as conduits for the transport of Brushy Canyon sands into the deep basin and as ponding areas where submarine fans were formed.

Similar patterns of thickness distribution can be seen on isolith maps of reservoir quality sandstone within the lower Brushy Canyon. One map, derived from density porosity logs, show the net thickness of sandstone with at least $15 \%$ porosity (Fig. 57). A map that shows thickness of sandstone with at least $10 \%$ porosity has similar patterns (Fig. 58). Both of these maps indicate the distribution of reservoir quality sandstones that are capable of sustained, economic levels of oil production, although the sandstones with a minimum of $10 \%$ porosity may be marginally productive. Reservoir quality sandstones in the Brushy Canyon that are oil-productive typically have porosity in the 12 to $22 \%$ range (see Asquith et al., 1996; Gawloski, 1995; Hoose and Dillman, 1995; LeMar, 1995; May, 1996; Mitchell, 1995; Tittl, 1995; White, 1995; Worrall, 1995). It is possible that some sandstones with more than $15 \%$ porosity will not be productive because of the dominance of micropores that that inhibit the migration and production of oil (see Asquith et al., 1996). 
Thicker areas of reservoir quality sandstones are located along the northern and western margins of the basin and are distributed along linear to lobate trends that extend from the basin margin into the deeper parts of the basin. Primary sources of reservoir quality sand were mostly located to the northwest and the west. The eastern part of the Northwest shelf contributed lesser, but still significant, volumes of sand. Only minor amounts of reservoir quality sand were derived from the Central basin platform to the east. An overlay of the $15 \%$ porosity isolith map on the Bone Spring structure map indicates that the reservoir sandstones are confined mostly to the structural depressions in the underlying Bone Spring or are present as lobe-shaped deposits (submarine fans) downslope of where the depressions terminate (Fig. 59). Deflection of transporting currents around structural closures and noses in the basin is evident in the map patterns.

Depositional sandstone units are separated by $5 \mathrm{ft}$ to $20 \mathrm{ft}$ thick layers of organicrich siltstones. These siltstones are represented on logs as radioactive beds. Most exhibit much greater lateral continuity than the interbedded sandstones. Several of the siltstone beds can be correlated throughout the basin. They are thought to represent periods of basin starvation (Garber et al., 1989; Gardner, 1997). The siltstones are use to internally subdivide the Brushy Canyon into its upper, middle, and lower units.

When areas of established, discovered oil production from the lower Brushy Canyon are compared with the structural configuration of the lower Brushy Canyon (Fig. 55), it is evident that oil accumulations are not coincident with the larger scale structural closures and noses. Productive areas are mostly coincident with structurally low areas in the Brushy Canyon. These low areas are inherited from paleobathymetric and paleostructural lows that characterize the upper surface of the underlying Bone Spring Formation. Areas of lower Brushy Canyon production are coincident with reservoir 
fairways where net thickness of lower Brushy Canyon sandstones with at least $15 \%$ porosity is $15 \mathrm{ft}$ or more (Fig. 57). The map of net thickness of sandstones with porosity greater than $10 \%$ exhibits a somewhat less explicit correlation with production (Fig. 58). Therefore, the fuzzy logic system probably should not emphasize structure in the conventional manner. Instead of focusing target areas on structural closures and noses, the fuzzy logic system will focus on paleostructural lows that acted as conduits for sediment transport. Structural closures may be significant where they are located downslope and acted to pond sediment flows and cause accumulation of thick reservoir quality sands, or where the structural configuration postdates sediment deposition and therefore had no affect on sediment transport routes.

A major question is raised by the maps that overlay productive areas on the reservoir sandstone trends. Why is production largely absent from areas in the westernmost and northwesternmost parts of the research area where reservoir sandstones occur in abundance? These areas typically have more than $100 \mathrm{ft}$ of lower Brushy Canyon sandstone with at least $15 \%$ porosity, yet these areas lack production. Although this may be partially due to incomplete testing and evaluation, it is likely that geological factors also play a role and that some parts of these sandstone-rich areas are truly barren of commercial hydrocarbons. Can this be explained by a paucity of seals? It may be that oil and gas have migrated updip out of the basin and have not been trapped in the Brushy Canyon for lack of adequate barriers to migration. Figure 57 shows that the larger oil accumulations within the Brushy Canyon coincide with areas of thick reservoir sandstones that thin or pinchout in an updip (northerly, northwesterly, or westerly) direction. The thin sands in the northernmost and westernmost parts of the basin appear 
to be continuously connected with the outcrop and therefore have not provided updip seals, at least along major sand trends.

Alternatively, eastward tilting during the Laramide may have led to flushing of the Brushy Canyon in this region by influent groundwaters (Lindsay, 2001). Influent waters may have moved downdip in an easterly direction until they encountered seals which also acted to trap hydrocarbons in stratigraphic traps to the east. Map that show salinity of produced lower Brushy Canyon waters (Fig. 60, 61) were constructed for this project and indicates that salinity decreases toward the northern and western margins of the basin indicating recharge of fresh water along the lower Brushy Canyon outcrop, and therefore supporting Lindsay's hypothesis.

Another possibility that may explain the sparse distribution of hydrocarbons in these areas is an absence or paucity of source rocks. It has been reasonably well established that, at least in places, oil accumulations within the Brushy Canyon are sourced by organic-rich siltstones within the Brushy Canyon (Hays and Tieh, 1992; Robinson, 1993). The sandstone reservoirs are interbedded with their source rocks and the source strata also act as seals for the accumulations. Robinson (1993) concluded that the low permeabilities of Brushy Canyon reservoirs are indicative of short migration distances. If this is the case, then oil accumulations should be preferentially concentrated in areas where source rocks are mature, thick, and have relatively high contents of organic carbon. Data and maps relating to these hypotheses were collected and created as part of this project in order to provide the fuzzy expert tool information with which to evaluate the importance of source rocks. A map of total organic carbon (TOC) content of lower Brushy canyon source rocks (Fig. 62) indicates that sufficient organic material is present within the lower Brushy Canyon to form adequate source facies throughout the 
Delaware Basin. Thermal maturity of Brushy Canyon source rocks, although adequate for oil generation throughout the research area, increases to the south in the deeper parts of the basin and also to the west with increasing proximity to the Rio Grande rift (Figure 63). Oil gravity data collected and mapped for this project indicate a bimodal distribution of lower Brushy Canyon oils (Fig. 64). Oils with higher API gravities (API $>40$ degrees which are lighter and presumably more thermally mature) have been trapped along major sandstone fairways whereas somewhat heavier oils (API $<40$ degrees) are present where sand bodies do not occur along major sandstone fairways. This distribution of oils suggests that lighter more mature Brushy Canyon oils may have been generated in deeper, more mature parts of the Delaware basin and migrated updip along sandstone fairways until they were trapped. The somewhat heavier oils had their origin in source beds close to the reservoirs in the shallower parts of the basin; the limited extent of sandstone bodies in these areas caused local hydrocarbon entrapment and prevented the oil from migrating longer distances. Thus, proximity to sandstone fairways plays a significant role in oil gravity and quality within the lower Brushy Canyon.

\section{Siluro-Devonian Carbonates}

Devonian and Siluro-Devonian carbonates produce from numerous oil and gas fields in southeastern New Mexico (Fig. 65). The 122 Siluro-Devonian fields in southeast New Mexico had produced a cumulative 443 MMBO by 1995 (Broadhead and Speer, 1995), that is, $10 \%$ of the oil produced from southeast New Mexico. Production is from a number of zones within the Silurian and Devonian sections (Fig. 66). A variety of mechanisms form traps, most notably anticlines, faulted anticlines, and subunconformity pinchouts (Speer, 1993). 
Geologic data acquisition continued on the Siluro-Devonian carbonates during the reporting period. Stratigraphic and structural data were correlated and compiled by Destini Baldonado, a graduate student in the Earth and Environmental Sciences Department at New Mexico Tech, assisted by Jason Lennane, graduate student, and Becky McGill, undergraduate student, who are also in the Earth and Environmental Sciences Department at New Mexico Tech. One thousand six hundred wells in southeast New Mexico that have penetrated the Devonian (Fig. 67) had been identified during the previous reporting period and entered into a database; longitude and latitude have been calculated from a digital land grid for these wells. During the present reporting period, a regional network of cross sections was constructed in order to establish correlation control and to provide quality assurance of data (Fig. 67). Logs from a total of 465 wells were correlated throughout southeast New Mexico (Fig. 68). Correlated wells were selected from all productive Siluro-Devonian fields and nonproductive areas as well in order to provide a dataset that fully contrasts productive areas with nonproductive areas. In each well, the top of the Siluro-Devonian carbonate section was correlated in addition to the top of the Fusselman Formation and the top of the Montoya Formation. Several prominent marker beds within the Siluro-Devonian section (Fig. 67) were correlated as well.

Similar to our earlier work on the Brushy Canyon Formation, we used our correlated data to produce geologic structure maps (Fig. 69), and isopach (thickness) maps (Fig. 70) of Siluro-Devonian carbonate strata. We also compiled thickness data for the eleven units that constitute the Siluro-Devonian carbonate sequence (Figs. 66 and SD3). A subcrop map that shows the Siluro-Devonian units that underlie the Woodford Shale was constructed (Fig. 71). This map indicates that strata that underlie the Woodford 
are truncated to the north and west by a regional angular unconformity. Of 48 productive wells examined thus far, almost half have produced from units 3, 4, and 5 within the Siluro-Devonian section (Fig. 72). We will relate these maps to production /nonproduction in both visual and artificial intelligence settings. The goal is to use our artificial intelligence system to predict trap configurations and locations in Devonian strata.

We began construction of regional maps of petroleum source rocks during the 2002-2003 reporting year. Assisting in this effort were Bryce Johnson, Lynsey Rutherford and Irene Roselli, undergraduate students at New Mexico Tech. The chief source rock unit is the Devonian Woodford Shale, which directly overlies the Devonian carbonates (Fig. 66). The Woodford is thought to be the chief source unit for lower Paleozoic reservoirs in the Permian (Hills, 1984). Strata that overlie the Siluro-Devonian carbonates north and west of the Woodford pinchout may also be source rocks and will be evaluated for source character during the next project year.

Existing source rock data were utilized where available (Broadhead et al., 1998). Because of a paucity of publicly available source rock data in the lower Paleozoic section, source-rock analyses were performed on 11 additional wells in order to provide a distributed network of data points throughout the basin (see Fig. 73 for data locations). The new source rock analyses were performed on well cuttings archived in the Subsurface Library of the New Mexico Bureau of Geology and Mineral Resources.

As with the Brushy Canyon, we have mapped regional distributions of source rock maturity for the Woodford. We plan to map distribution of source-rock quality and relate these to oil and gas distribution. The top and base of the Woodford Shale were correlated in 514 wells during the most recent project year. These data will be used to 
map Woodford structure, thickness, and extent. Because the Woodford acts as the vertical seal on most oil and gas accumulations in the Siluro-Devonian carbonates, its thickness and extent is of interest not only in its role as a source rock but also in the role it plays in trap formation.

With the depth of the Woodford varying from less than $7000 \mathrm{ft}$ in the northern part of the basin in Chaves County to more than 15,000 $\mathrm{ft}$ in the southern part in Lea and Eddy Counties, we have encountered thermal maturity variations across the oil window/gas window boundary that relates to the distribution of oil reservoirs and gas reservoirs (Fig. 73). The map of Siluro-Devonian oil and gas fields (Fig. 65) indicates that most gas fields are located in the south-central parts of the basin where thermal maturity of source rocks is higher. The higher thermal maturity relates generally to structural depth, but not entirely. These relationships will be quantified for use in the artificial intelligence system. Thermal maturity variations may also help in the prediction of gas-oil ratios and therefore relate to aspects of recovery efficiency.

Total organic carbon (TOC) values were also mapped for the Woodford (Fig. 74). This map indicates the Woodford contains sufficient TOC to be a good source rock throughout the area, but TOC content does decrease significantly toward the northern edge of the basin where the Woodford is also thin. During the next project year, we will combine TOC with other source rock parameters such as thickness and maturity to produce maps of generative potential that are anticipated to be related to the distribution of hydrocarbons in the Siluro-Devonian section.

\section{$\underline{\text { Experimental }}$}

There are no experiments associated with this project. 


\section{$\underline{\text { Oil Price Forecast }}$}

Future oil price is the fundamental parameter in assessing the risk of any oil or gas production venture. One aggregate of speculation by experts on the purchasing and selling of crude oil is the Goldman Sachs future's price of oil. These experts try to reasonably predict the price of a barrel of oil a month in advance. Shown in Fig. 51 is the 30-day average of these experts' predictions preceding the strike date. The average is compared to the Navajo refinery posted price of West Texas Intermediate on the strike data. Notice that from June 1996 through October 1997 the experts did an excellent job. However, from November 1997 to March 1999 the error is approximately 33\% or about $\$ 5 / \mathrm{bbl}$ more than the posted price. Also from May 1999 to December 2000 the error is about $25 \%$ or $\$ 7 / \mathrm{bbl}$ less than the posted price. The predictions presented in Fig. 51 are crossplotted in Fig. 52. Notice that $\mathrm{R}^{2}$ is 0.72 , yielding an $85 \%$ overall correlation coefficient. It might prove fruitful to investigate other future's estimates to see if they can more accurately predict the future price of oil.

The future price of commodities other than oil is also the subject of speculation by experts. The 90-day future price of the Euro vs. the Yen was examined using crossplots similar to the oil futures versus posted price, the hope being that Euro/Yen traders might indirectly have insight to the future price of oil. Figure 53 shows the crossplot of the WT Posted Price of oil vs. the 90-day average of the Euro/Yen. Based on the $\mathrm{R}^{2}$ of 0.96 (98\% correlation coefficient) there appears to be a strong relationship between the Euro/Yen future price and the posted price of WT intermediate, at least for this small dataset.

The relationships between the three-month future price of the Euro/Pound, 30year Treasuries, 10-year Treasuries, and the Future Dow Jones with the posted price of 
WT intermediate oil were also examined in a similar manner. The results based on the goodness of the best-fit line are summarized in Table 7.

Table 7. Goodness of the Relationship between Commodity Future Price and Oil Price

\begin{tabular}{|l|l|}
\hline Commodity & Correlation Coefficient, \% \\
\hline Goldman Sachs future price of oil & 85 \\
\hline Euro/Yen & 98 \\
\hline Euro/Pound & 96 \\
\hline 30-year Treasuries & 94 \\
\hline 10-year Treasuries & 94 \\
\hline Future Dow Jones & 93 \\
\hline
\end{tabular}

The results of this preliminary analysis, albeit with a small dataset, suggests that the future price of some commodities other than oil may be useful when predicting oil price. Assuming that the correlation coefficient describes experts' ability to forecast the future, it appears that the futures price of the Euro/Yen and Euro/Pound are closely tied to the price of oil. This is not as surprising as one might think, as both the Euro and Yen (for example) are currencies for nations and regions which on a whole are dependent on oil imports to fuel their industries, and hence their economies.

A strong concern, however, is that these futures may be strongly affected by many of the same factors that affect the accuracy of futures prices and therefore break down prior to periods of change, such as a sudden war, or other unexpected event.

Ultimately most users will likely prefer to use their own corporate philosophy when adding the economic impact of future oil price to the Risk assessment. This will be a necessary feature of the final system.

\section{Technology Transfer}

During this twelve-month period (April 2002-March. 2003) the following eleven papers or presentations were made to disseminate the results of the project: 
1. Balch, R.S., Weiss, W.W., and Ruan, T.: Simulated Expert Interpretation of Data to Predict Drilling Risk on a Regional Scale, Case Study -- Brushy Canyon Formation, Delaware Basin, New Mexico in The Permian Basin: preserving our past - securing our future: West Texas Geological Society, Publication No. 02111, pp. 55-69.

2. Balch, R.S., Hart, D.M. and Weiss, W. W.: "Regional Data Analysis to Better Predict Drilling Success: Brushy Canyon Formation, Delaware Basin New Mexico," paper SPE 75145 presented at the 2002 Symposium on Improved Oil Recovery, Tulsa, April 13-17.

3. Balch, R.S., Hart, D.M., Weiss, W.W., Broadhead, R.F.: "Using Artificial Intelligence to Predict Drilling Success Using Regional Data, Brushy Canyon Formation, Delaware Basin, New Mexico," Transactions, Southwest Section A.A.P.G Convention, Ruidoso, NM June 6-8, 2002.

4. Baldonado, D., and Broadhead, R., 2002, Preliminary investigation of the regional stratigraphy of Siluro-Devonian carbonates, Tobosa Basin, New Mexico, in The Permian Basin: preserving our past - securing our future: West Texas Geological Society, Publication No. 02-111, pp. 55-69.

5. Baldonado, D., and Broadhead, R., 2003, Preliminary investigation of the regional stratigraphy of Siluro-Devonian carbonates, Tobosa Basin, New Mexico (abstract): Transactions Southwest Section AAPG Convention, Fort Worth, Texas, CD-ROM.

6. Lennane, J., 2002, Controls on the accumulation of hydrocarbons in the lower Brushy Canyon Formation, southeastern New Mexico: M.S. thesis, New Mexico Institute of Mining and Technology, $147 \mathrm{p}$.

7. Lennane, J., and Broadhead, R.F., 2002, The hydrocarbon producing rocks of the lower Brushy Canyon (abstract), in Transactions Southwest Section AAPG Convention: Roswell Geological Society, p. 91.

8. Subramaniam V., and Weiss, W.W.: "Evaluation of Well Completion Opportunities in the Lower Brushy Canyon Using Neural Networks," Transactions, Southwest Section A.A.P.G Convention, Ruidoso, NM June 6-8, 2002.

9. Weiss, W.W.: "Reducing the Risk of Installing a Waterflood," Presented at the Annual Petroleum Engineering Summer School, Workshop No. 11 Risk Minimization and Probabilistic Application in the Petroleum Industry, Dubrovnik, Croatia, 10-14 June, 2002.

10. Weiss, W. W., Gottumukkala, V., and Balch, R.S.: "A New Method of Calibrating Wireline Logs With Carbonate Core Measurements to Recognize Pay Zones," Paper 77330 presented at the SPE Annual Technical Conference, San Antonio, TX Sept 29, 2002.

11. Weiss, W. W., Balch, R.S., and Stubbs, B.A.: "How Artificial Intelligence Methods Can Forecast Oil Production," paper SPE 75143 presented at the 2002 Symposium on Improved Oil Recovery, Tulsa, April 13-17.

Additionally, five companies have asked specifically for information on areas of the Predicted Potential map. Zooms of these areas were generated for Strata Production 
Company, Bass Enterprise Production Company, Reed and Stevens Inc., Speerex Ltd., and Lynx Petroleum Consultants Inc.

\section{Problems Encountered}

The acquisition of regional seismic lines continues to be a problem due to the value of the data. Local datasets are available such as those from the DOE-funded Nash Draw project. The processed data from this 3D data set was used to develop new methods of interpreting the distribution of thickness, porosity, water saturation and depth throughout the survey area. The methodology can be applied throughout the Delaware Basin.

Personnel changes at the Petroleum Recovery Research Center required changing the Project Manager to Dr. Robert Lee and the PRRC PI to Dr. Robert Balch. The transition has been smooth and no delay or changes were made to the project or its timeline.

\section{Next Year's Tasks}

\section{Continue Expert System Development}

Currently the Expert System has been programmed to use about 100 rules in several different categories. The current system can be run on a laptop computer and will be of great assistance in giving human explorationists a feel for the system. Work is ongoing in numerically grading questions, and interviewing Experts in both Brushy Canyon, and Devonian prospecting. It is anticipated that a fully functional system for the Brushy Canyon will be running before the next semi-annual report and the PRRC is organizing a large event this summer to showcase the system to Producers. This will coincide with the Fee Tool annual Symposia. 


\section{$\underline{\log \text { Analysis }}$}

As Devonian log information becomes available it will be correlated with core data to develop pseudo-logs. Production will then be correlated with the pseudo-log statistics.

\section{Geology}

During the next project year, we will finish acquisition of all geologic data. This will include:

1. Obtaining a limited number of source rock analyses on post-Woodford source facies that directly overlie the Siluro-Devonian carbonates north and west of the Woodford pinchout in order to assess their contributory role as a source for oil and gas in the Siluro-Devonian carbonates in the northern and western reaches of the basin.

2. Construct source-rock attribute maps of possible post-Woodford sources identified as a result of step 1 , above.

3. Construct a worms-eye map of strata that overlie the uppermost surface of the Siluro-Devonian carbonates.

4. Use Woodford data to produce an isopach map of the Woodford Shale.

5. Use Woodford thickness and source rock data to produce maps related to the generative potential of the Woodford.

6. For each of the Siluro-Devonian oil and gas fields, produce a map that indicates the Siluro-Devonian stratal unit that is the primary productive unit in that field.

7. Produce isopach maps of the major productive Siluro-Devonian stratal units and relate them to structure, stratigraphy, source rocks, and oil and gas production. 
8. Relate source rock thermal maturity to gas-oil ratios in Siluro-Devonian carbonate reservoirs.

\section{Conclusions}

Substantial progress has been made towards a finished Expert System that will run remotely from a browser on nearly any computer and be able to aid in development and drilling decisions for both the Brushy Canyon and Devonian plays by providing readily accessible public information that simulates an "Expert" opinion of a prospect in a short time, to enhance the work of a human explorationist.

The emphasis during the April 2002 through March 2003 period was directed toward Silurian-Devonian geology, development of rules for the fuzzy system, and online software. Four major categories of regional data (gravity, aeromagnetic, structure, and thickness) were selected, compiled and finalized. Regional gravity surveys cover the entire area of the Delaware basin and have been compiled with an accuracy of a few milligals.

We have generated a number of new and useful tools and technologies to support construction of the Expert System, including online useable interfaces for neural network analysis (PredictOnline), ranking of potential inputs using fuzzy logic (FuzzyOnline), a preliminary Expert System able to make rudimentary drilling decisions, and a web interface for accessing the databases and Expert System software.

Finally, this year's research has resulted in eleven papers that were presented at meetings. 


\section{References}

Balch, R.S., Stubbs, B.S., Weiss, W.W., and Wo, S.: "Using Artificial Intelligence to Correlate Multiple Seismic Attributes to Reservoir Properties," paper SPE 56733 resented at the 1999 SPE Annual Technical Conference, Houston, Oct. 3-6.

Broadhead, R.F., and Speer, S.W. (1995): Oil and gas fields in the New Mexico part of the Permian Basin, in: A Symposium of the Oil and Gas Fields of Southeastern New Mexico, 1995 supplement: Roswell Geological Society, p. 32-49.

Broadhead, R.F., Wilks, M., Morgan, M., and Johnson, R.E., 1998, The New Mexico petroleum source rock database: New Mexico Bureau of Mines and Mineral Resources, database DDS DB2, CD-ROM.

Hill, C.A., 1996, Geology of the Delaware Basin, Guadalupe, Apache, and Glass Mountains, New Mexico and west Texas: Permian Basin Section SEPM Publication No. 96-39, 480 p.

Hill, J.M., 1984, Sedimentation, tectonism, and hydrocarbon generation in Delaware Basin, west Texas and southeastern new Mexico: American Association of Petroleum Geologists, Bulletin, v. 68, p. 250-267.

Lin, Y., Cunningham, G.A., Coggeshall, S.V., and Jones, R.D.: "Nonlinear System Input Structure Identification: Two Stage Fuzzy Curves and Surfaces," IEEE Transactions on Systems, Man, and Cybernetics-Part A: Systems and Humans (1998) 28, No. 5, 678-684.

Lin, Y., and Cunningham, G.A.: "A New Approach to Fuzzy-Neural System Modeling," IEEE Transactions on Fuzzy Systems (1995) 3, No 2, 190-198.

Speer, S.W., 1993, PP-6: Siluro-Devonian, in Atlas of Major Rocky Mountain Gas Reservoirs: New Mexico Bureau of Mines and Mineral Resources, p. 163. 


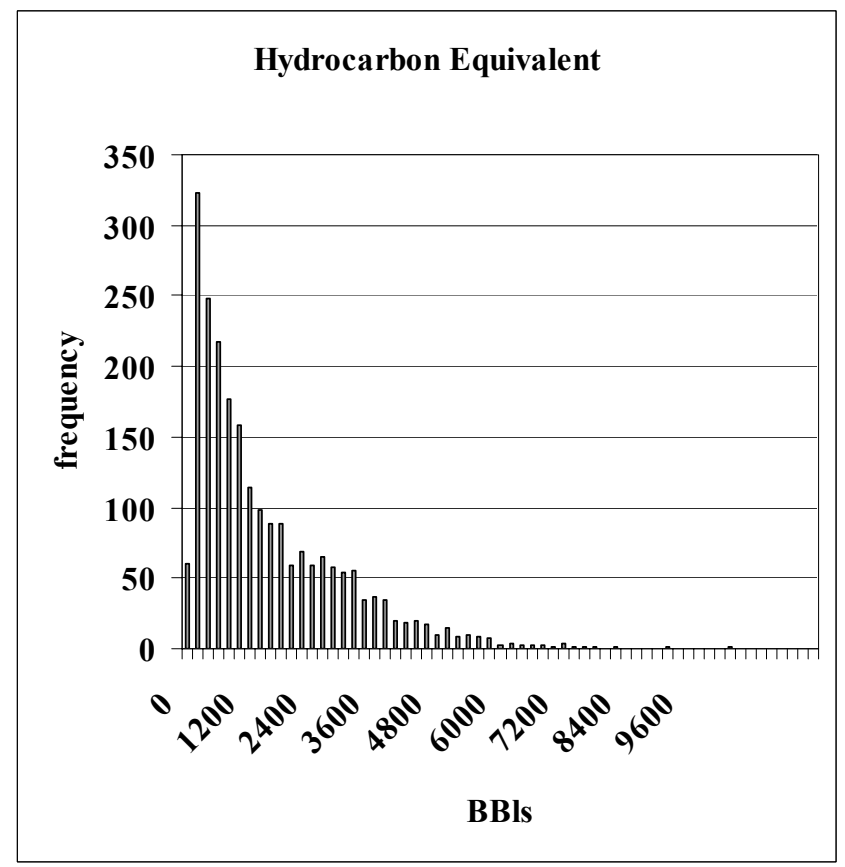

Fig. 1. Histogram of average hydrocarbon equivalent. Value is average monthly production over the first year of production for Brushy Canyon wells.

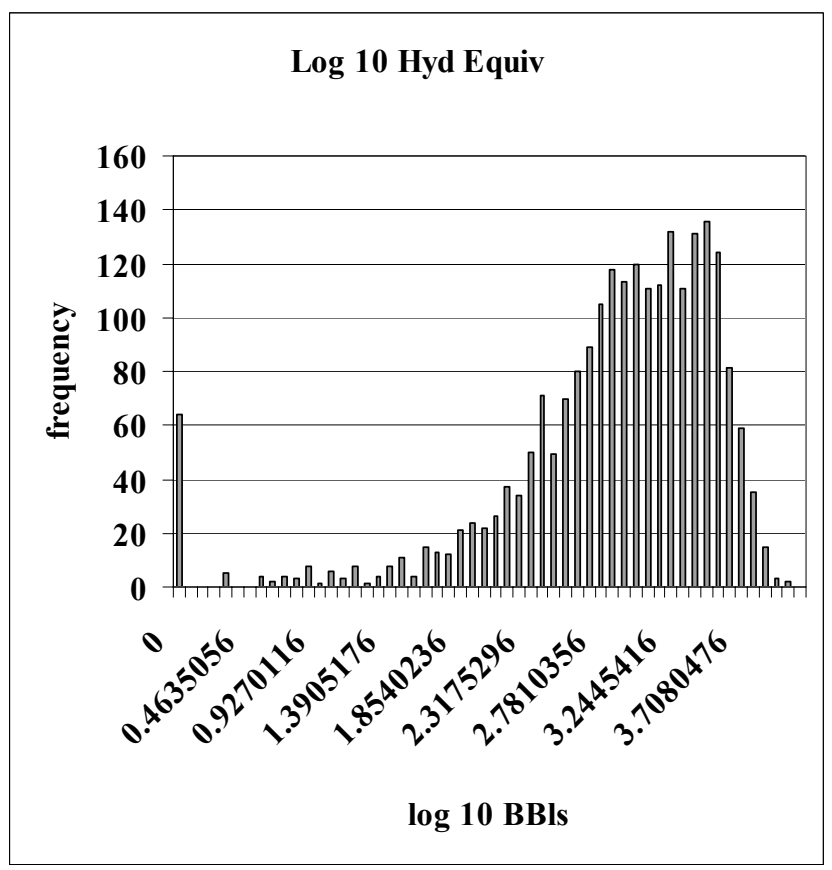

Fig. 2. Log filtered version of histogram from Fig. 1. Notice that the data is much better behaved and follows a rough gaussian curve. It is easier to see outliers with the data in this format. 


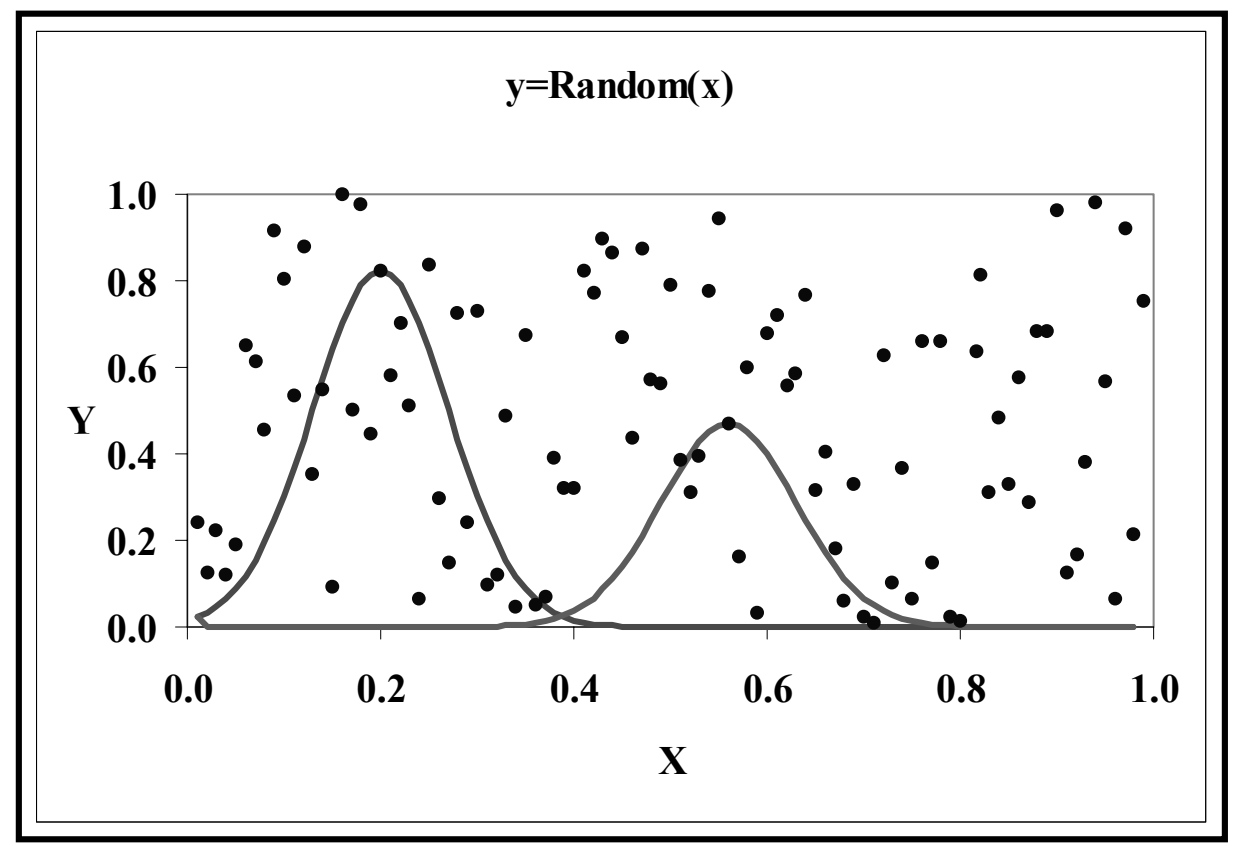

Fig. 3. Sample fuzzy membership functions drawn for two points of a 100-point random data distribution.

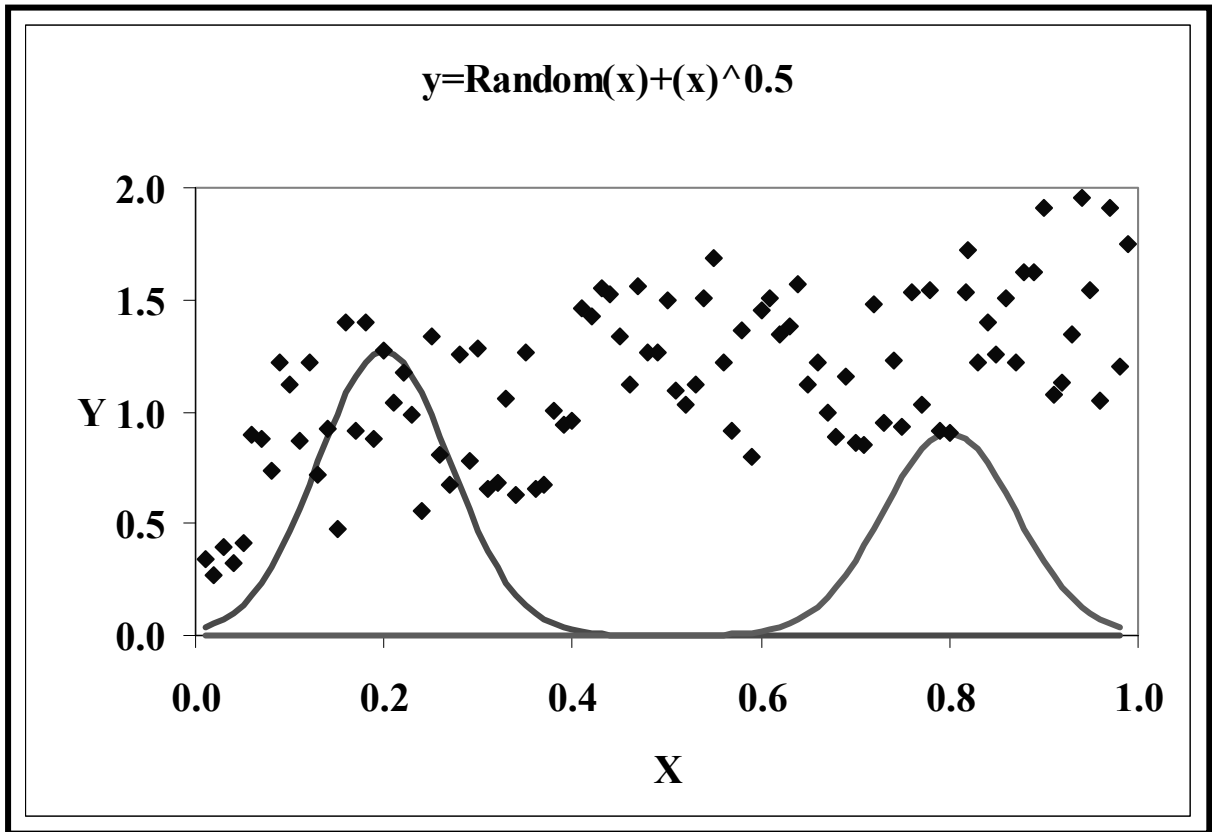

Fig. 4. Sample fuzzy membership functions drawn for two points of a 100-point random data distribution with a trend added. 


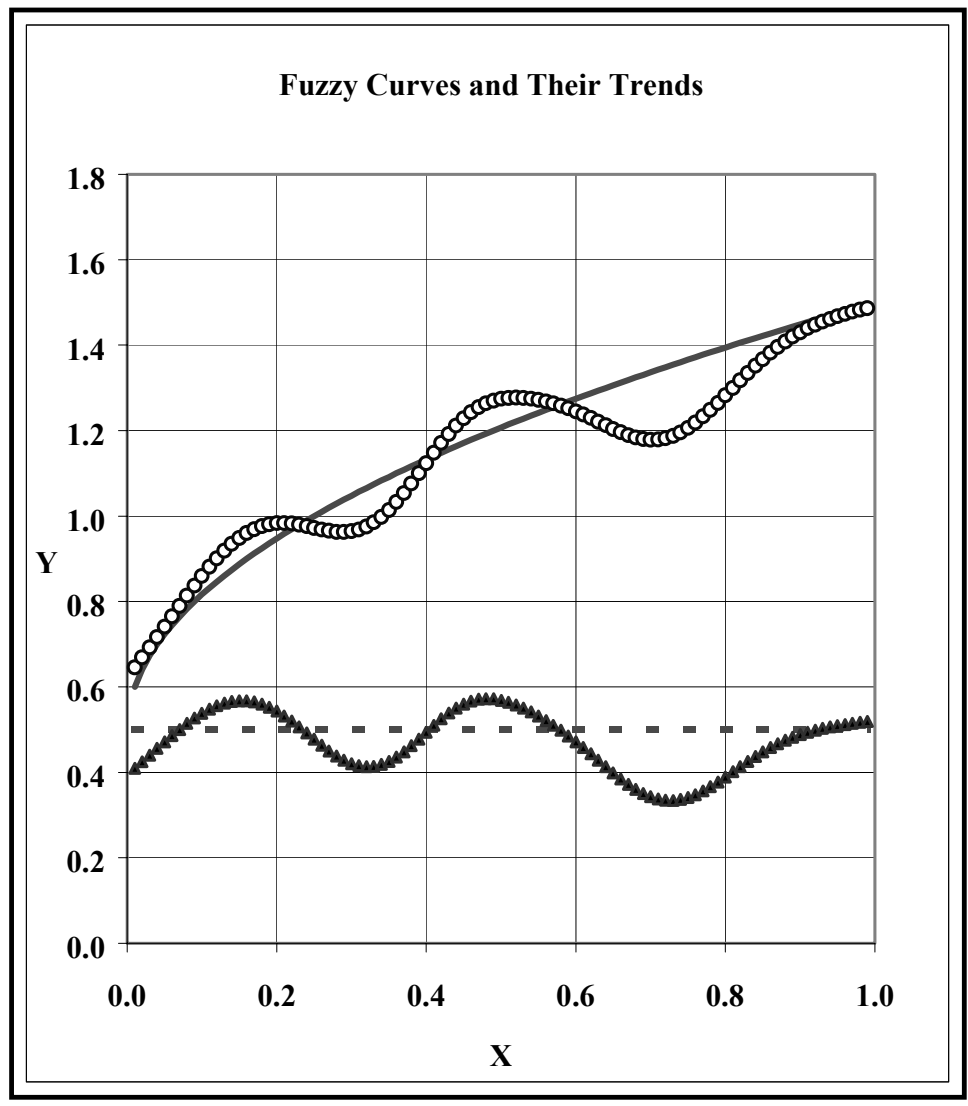

Fig. 5. Fuzzy curves for the data sets in Figs. 3 and 4. Fuzzy curves are drawn by doing a weighted sum of all the fuzzy membership functions. 
a) Input: gravity $d x$-grav dy-grav

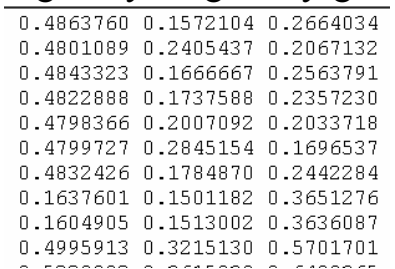

b) Processing

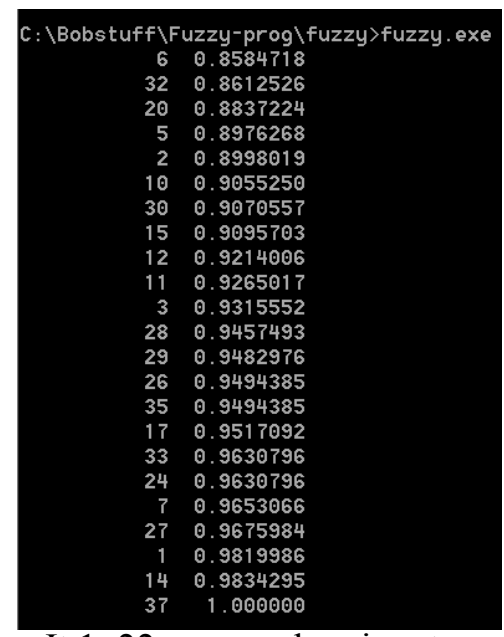

It 1: 22 non-random inputs

(14 random inputs

\begin{tabular}{c}
. .36 production \\
\hline 0.0988196. \\
0.0725581 \\
0.1568209 \\
0.1901703 \\
0.406621 \\
0.0688730 \\
0.368543 \\
0.2306216 \\
0.0137803 \\
0.1320701
\end{tabular}

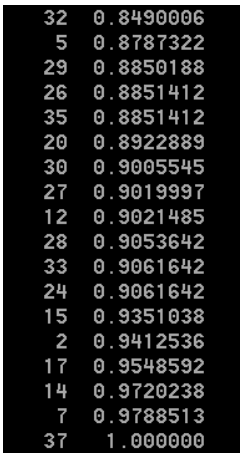

It 2: 17 nonrandom inputs independent of \#6 c) Results

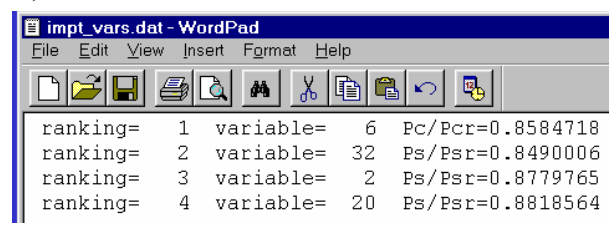

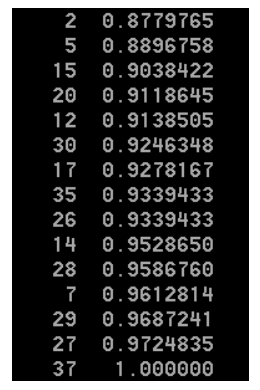

It 3: 14 nonrandom inputs independent of \#6 and \#32

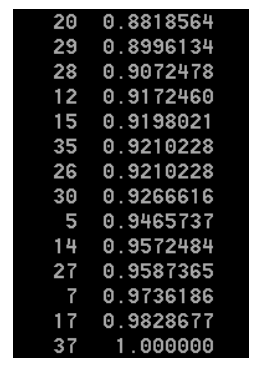

It 4: 13 nonrandom inputs independent of $\# 6$, \#32, and \#2

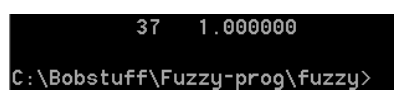

It 5: no non-random independent variables

Fig. 6. Operation of second stage fuzzy ranking program FuzzyOnline. A) is a sample input file. In this case we wish to correlate to each of 36 data found in the database for 520 wells and the average monthly production we wish to correlate to. B) shows a progressive reduction in the number of possible inputs (initially 36) through several iterations. In each iteration the best fitting variable is identified, then the rest of the data is studied for uniqueness with respect to that best variable. In this case five iterations identified the four strongest and unique inputs. C) The generated output file shows the strength and ID number of the best inputs for regression analyses 


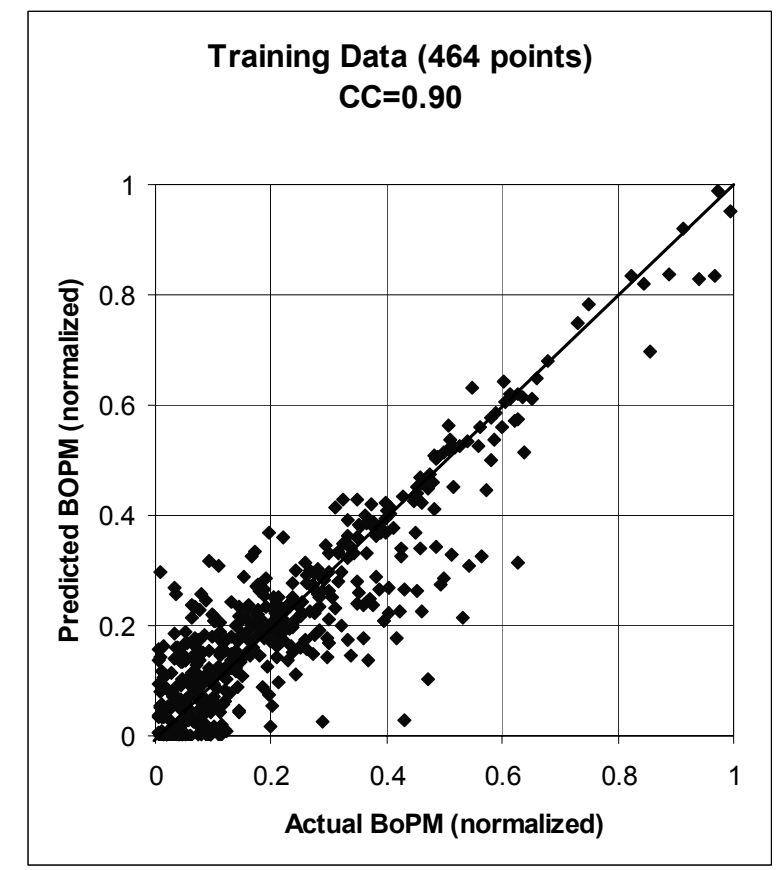

Fig. 7. Crossplot of neural network training using the inputs identified via FuzzyOnline.

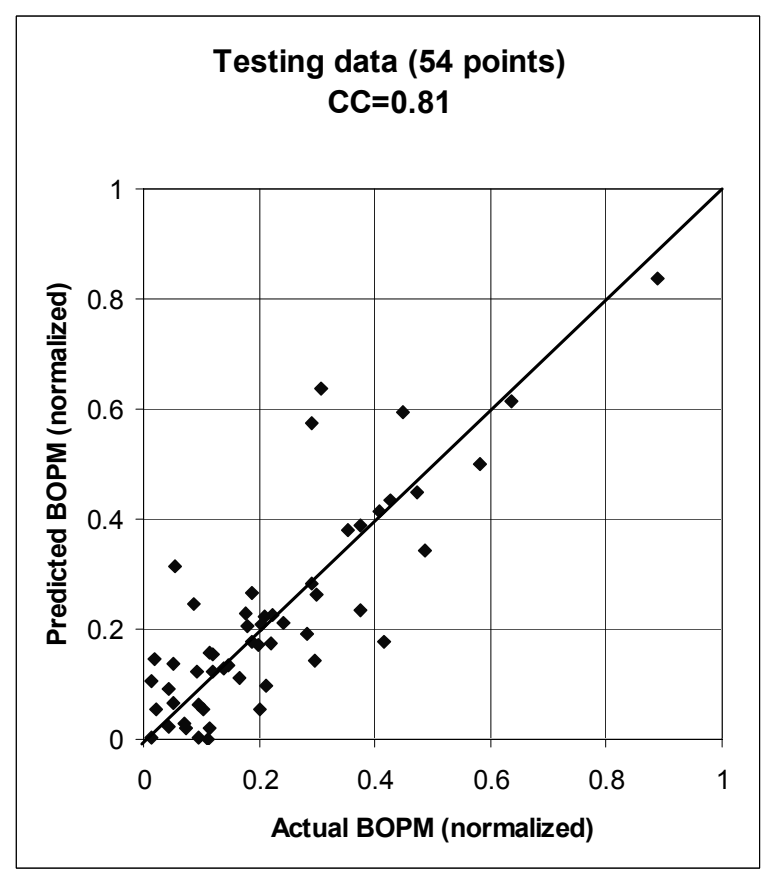

Fig. 8. Crossplot of testing data for the trained neural network of Fig. 7. 


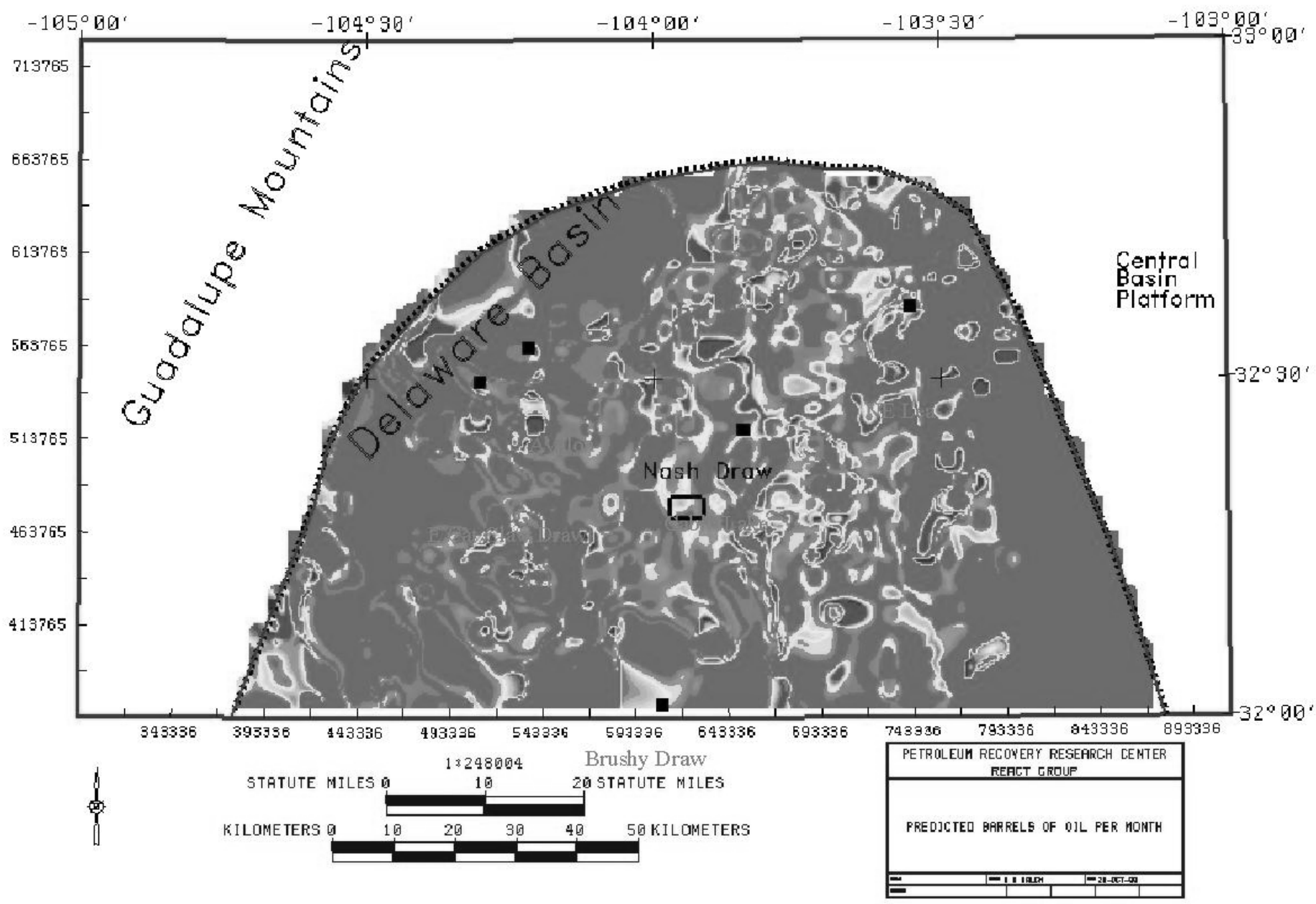

Fig. 9. Map of predicted production potential based on the trained and tested neural network regression. 


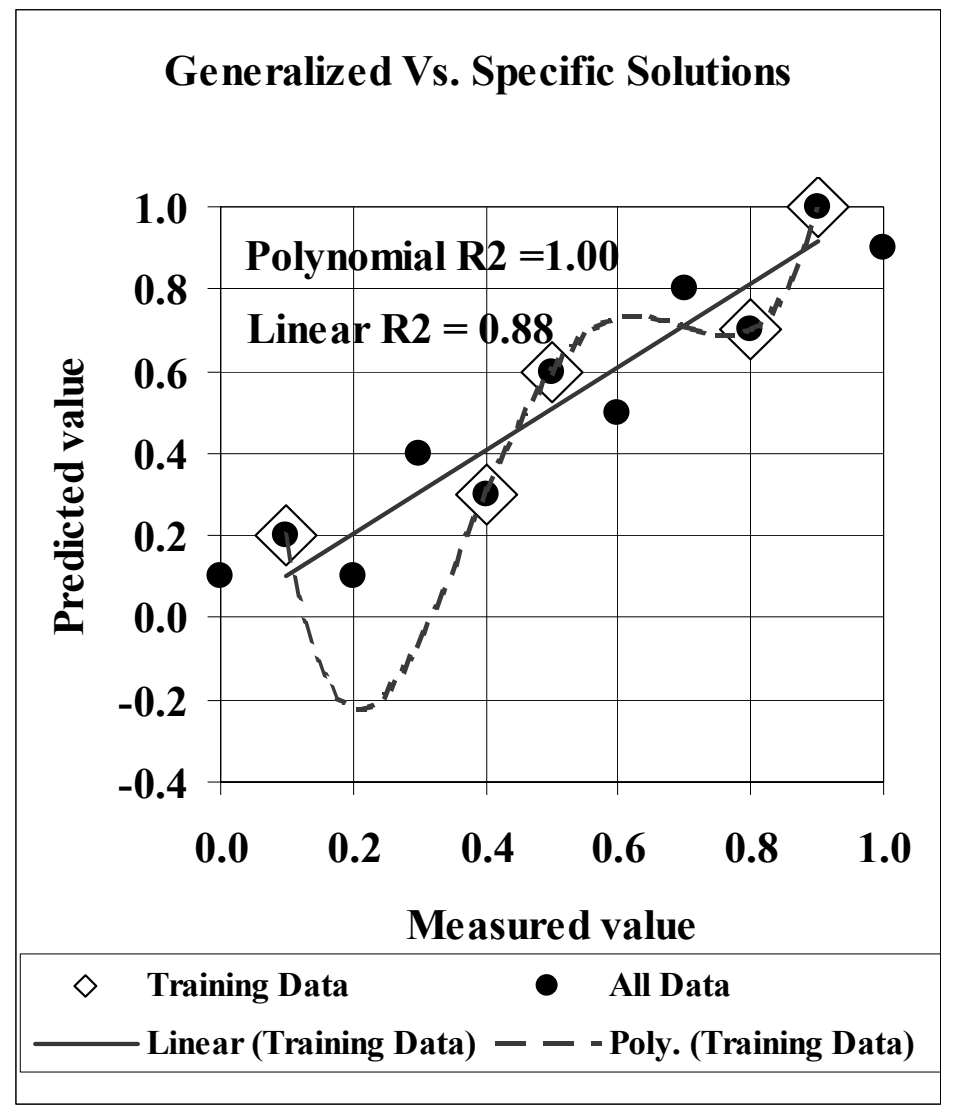

Fig. 10.This figure demonstrates the benefits of neural network regressions for complex problems where forecasts need to be made. Assuming only the five points enclosed by diamonds are known to start, it is quite easy to fit a polynomial that exactly fits the training data but poorly fits the true data distribution. Neural networks can bypass this problem if a sufficient sample exists for training by creating a generalized solution. 


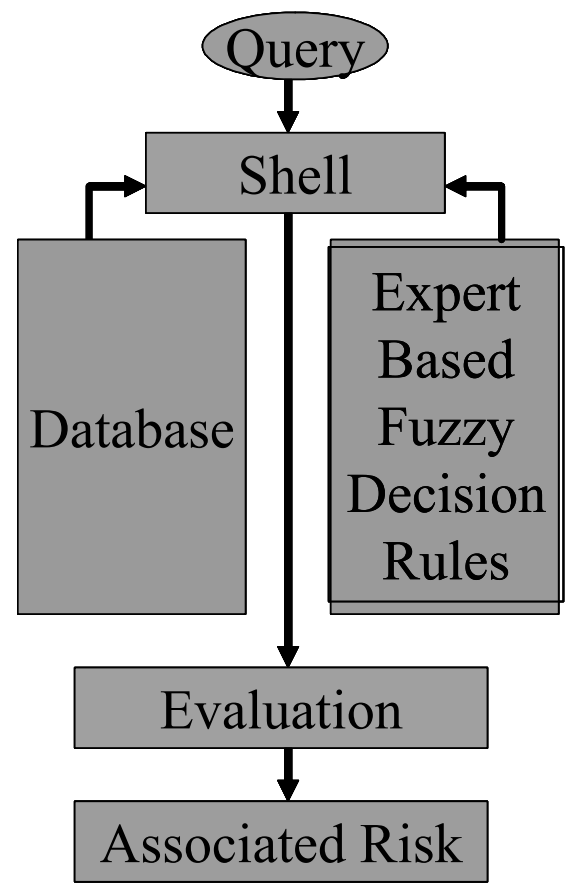

Fig. 11. The original schematic for the fuzzy expert system shell.

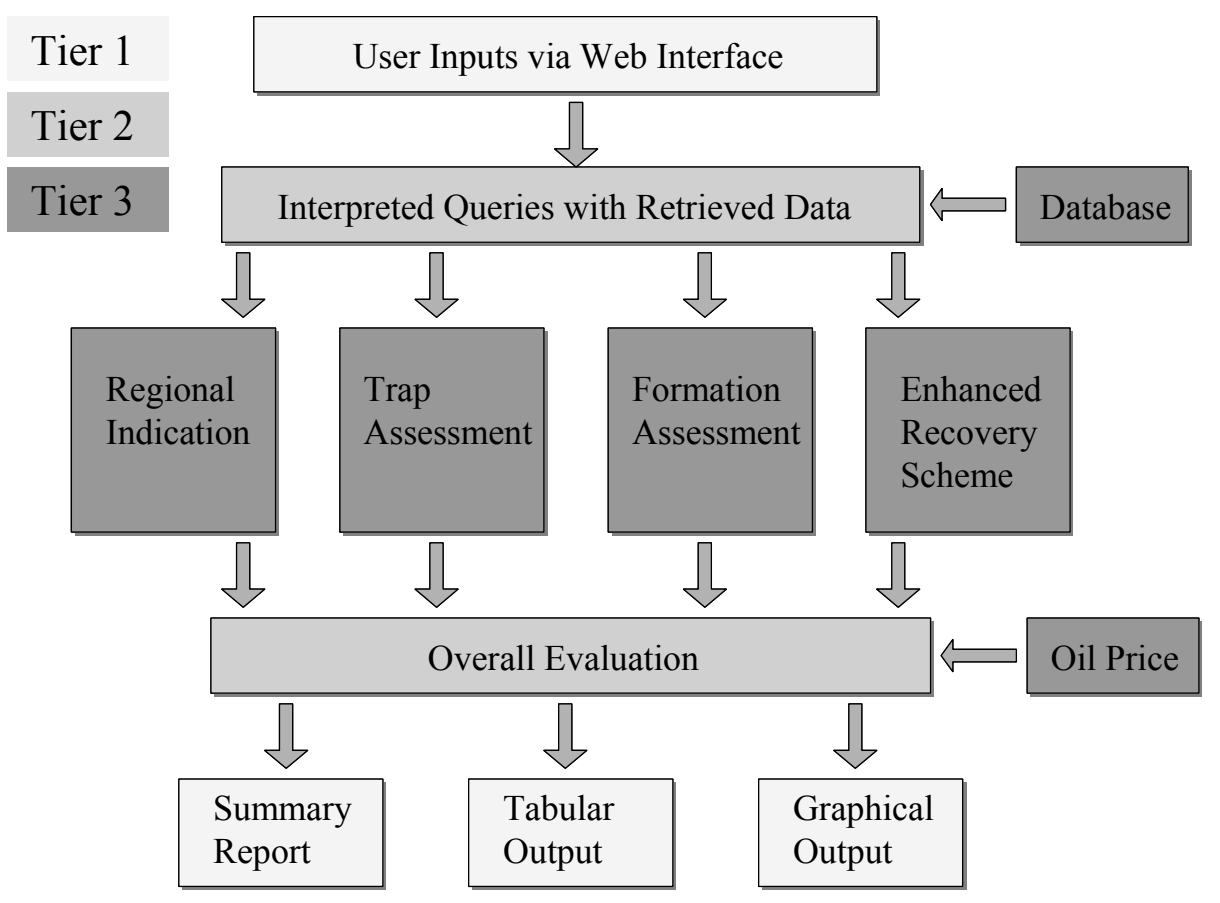

Fig. 12. More complicated system, which breaks the analysis into several separate categories to simplify calculations and customization. 


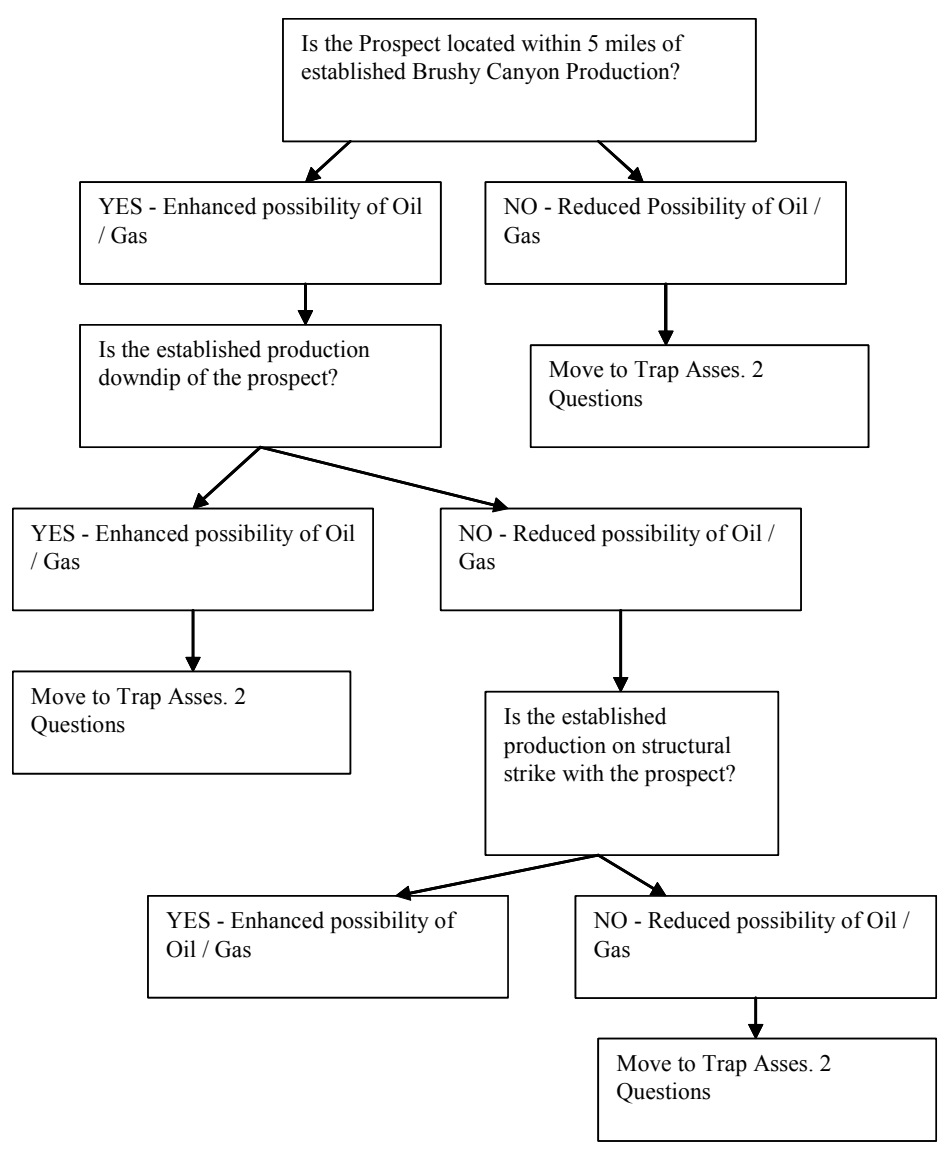

Fig. 13. Initial rules for trap assessment (Part I).

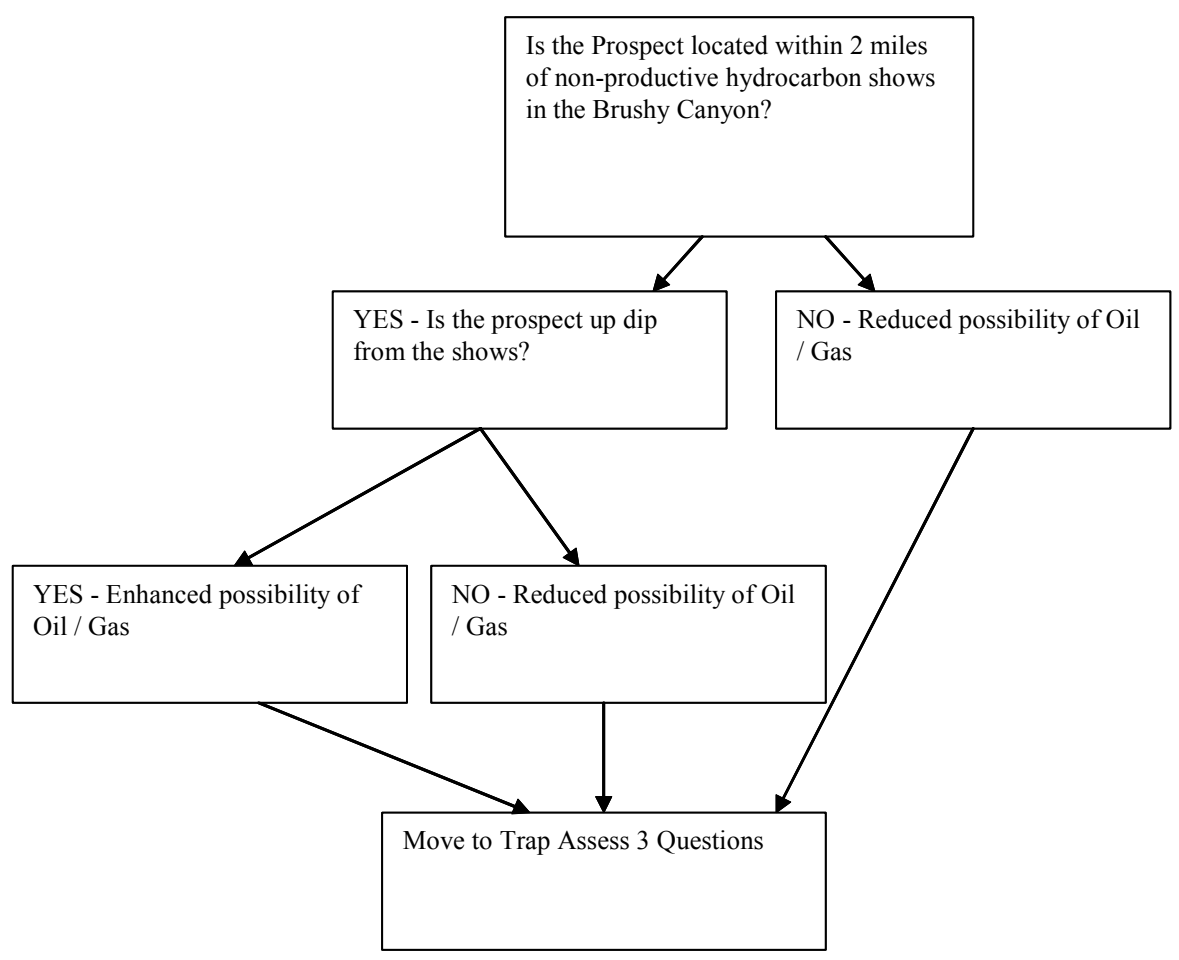

Fig. 14. Initial rules for trap assessment (Part II). 


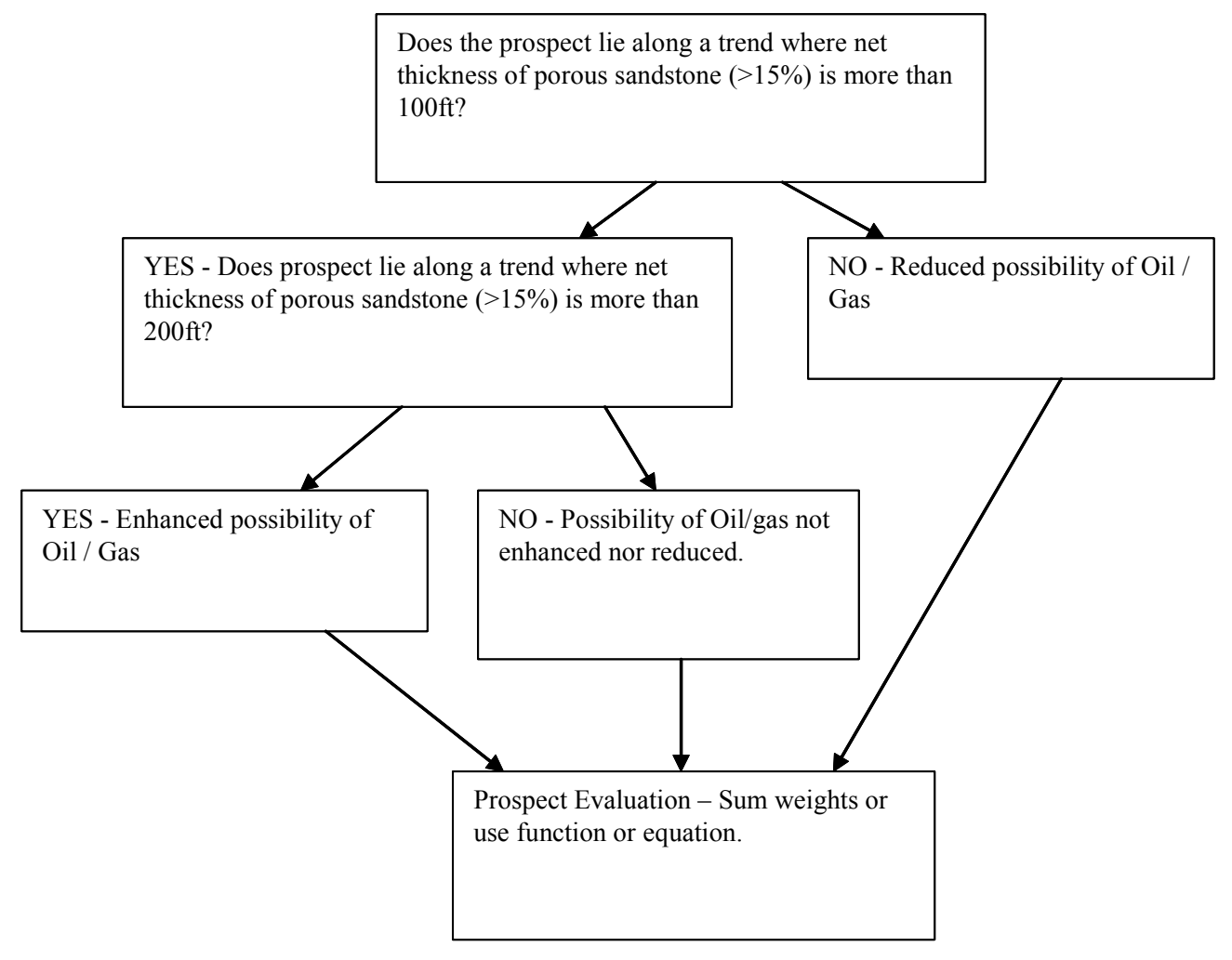

Fig. 15. Initial rules for trap assessment (Part III).

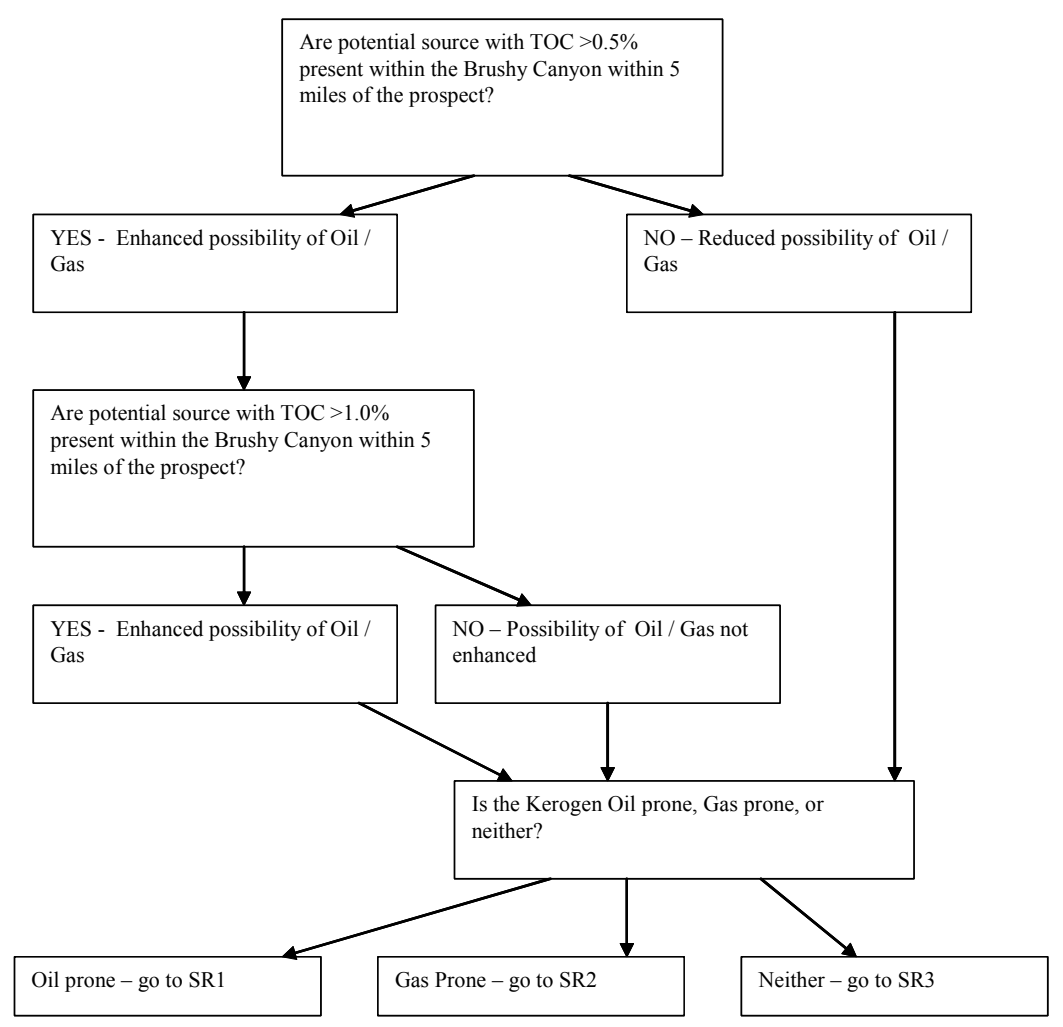

ig. 16. Initial rules for source rock assessment (Part I). 


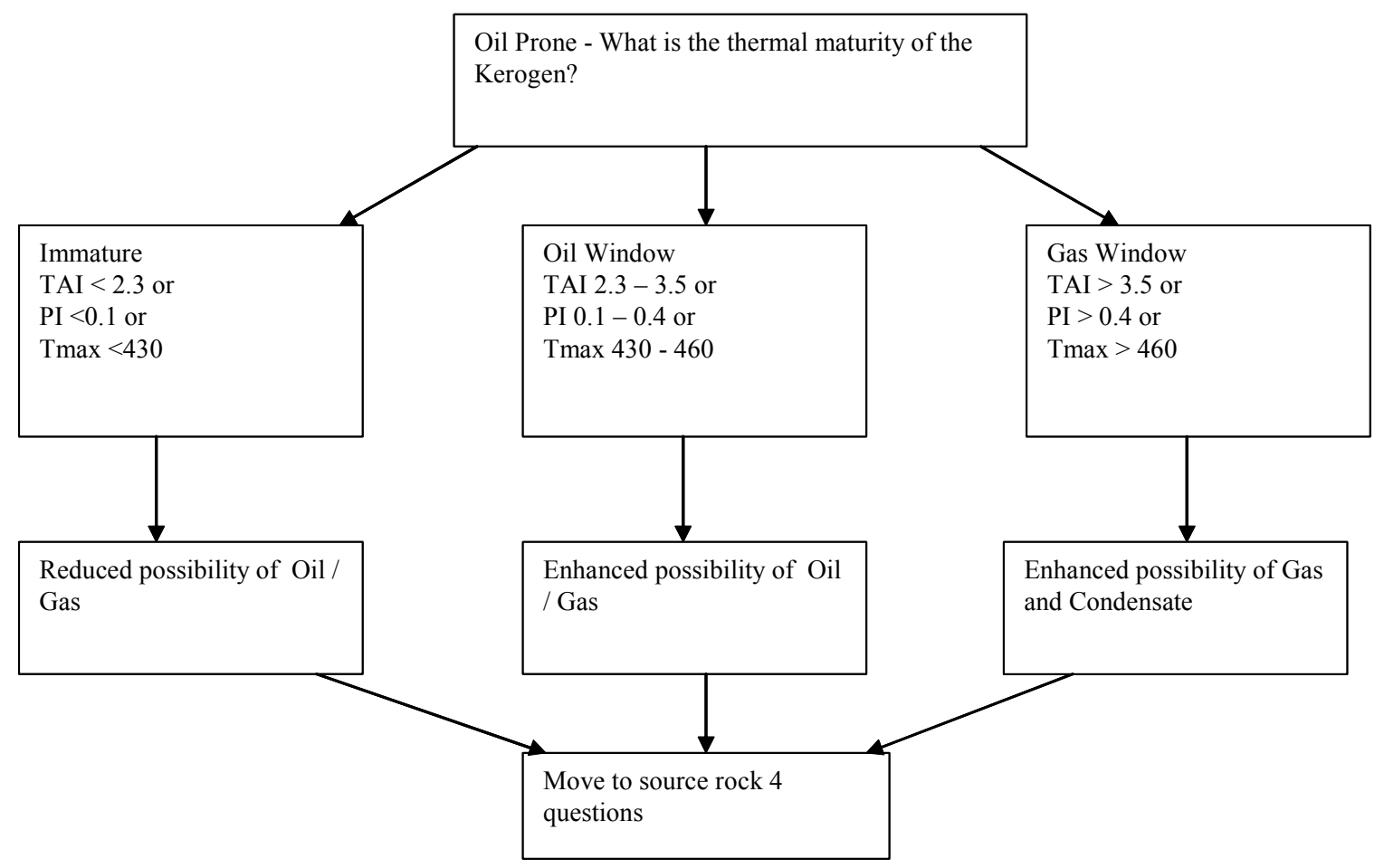

Fig. 17. Initial rules for source rock assessment (Part II).

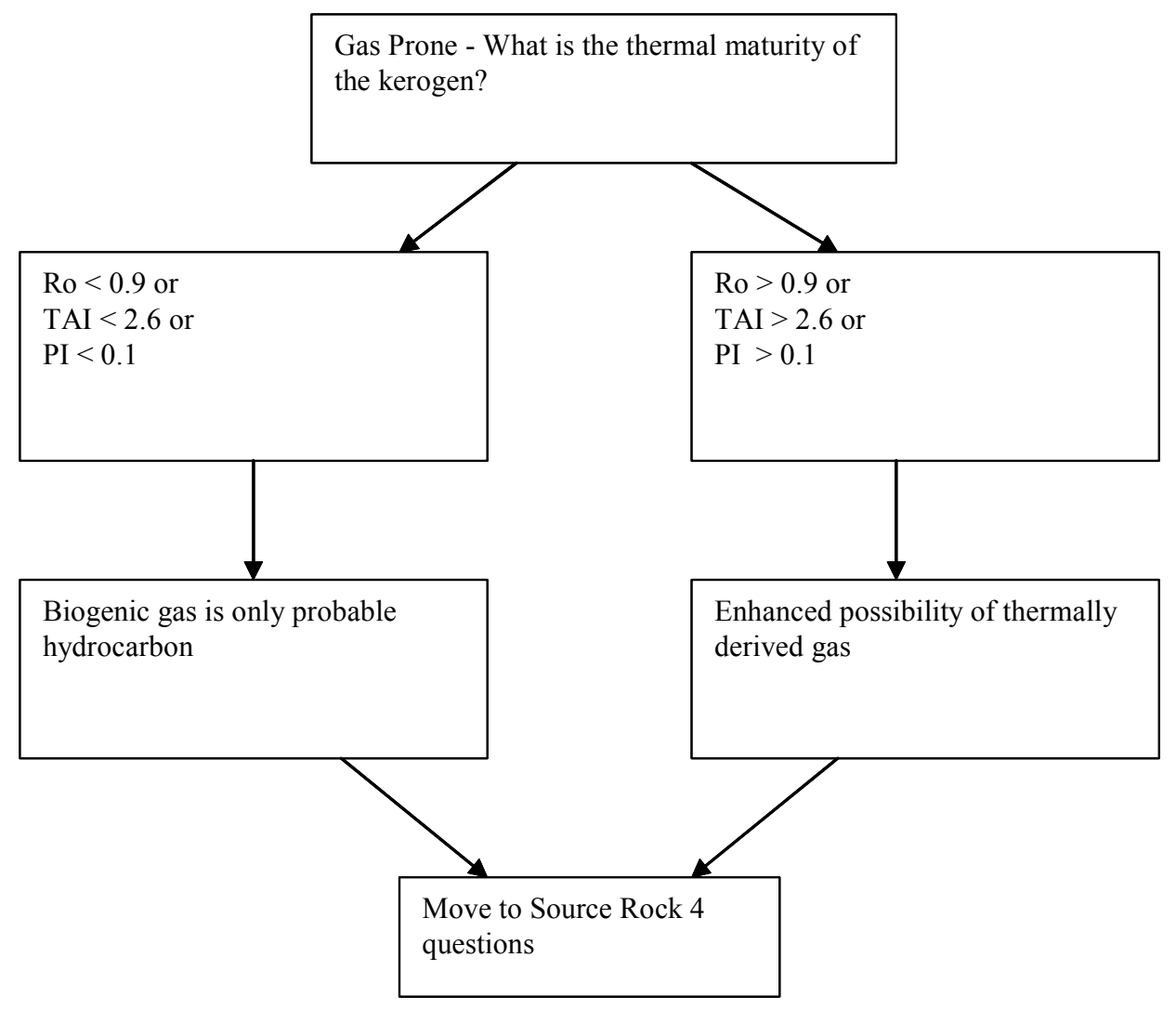

Fig. 18. Initial rules for source rock assessment (Part III). 


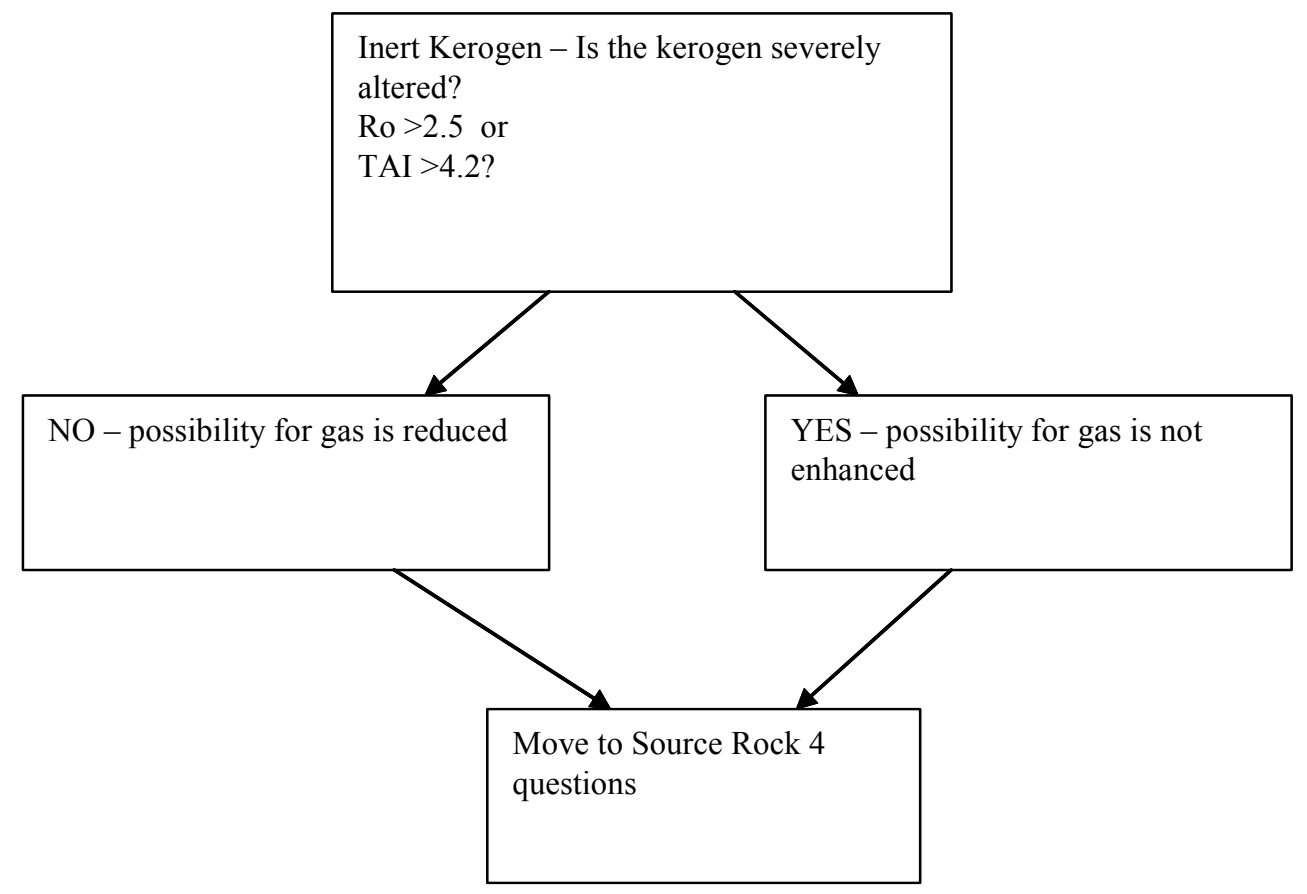

Fig. 19. Initial rules for source rock assessment (Part IV).

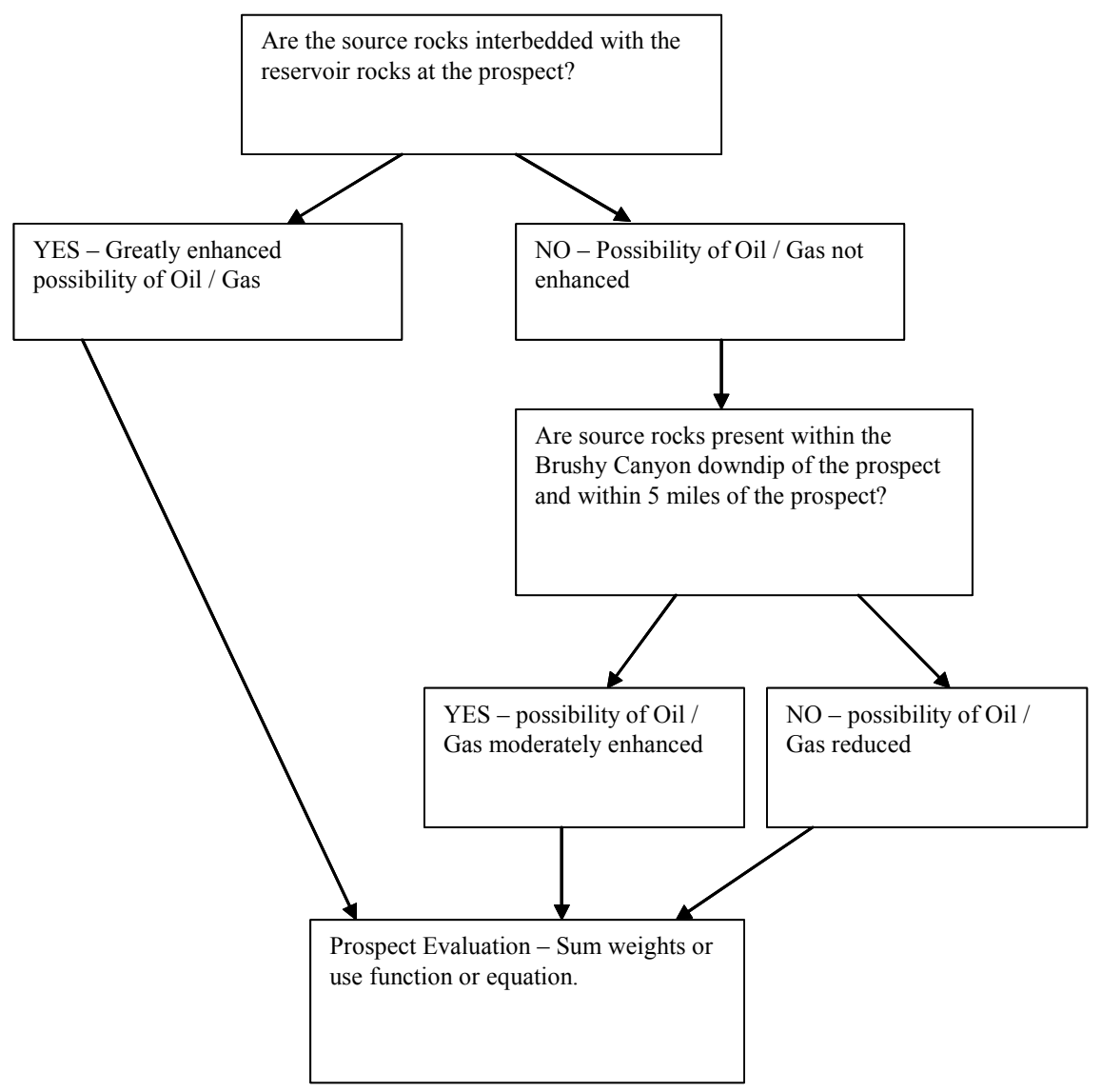

ig. 20. Initial rules for source rock assessment (Part V). 


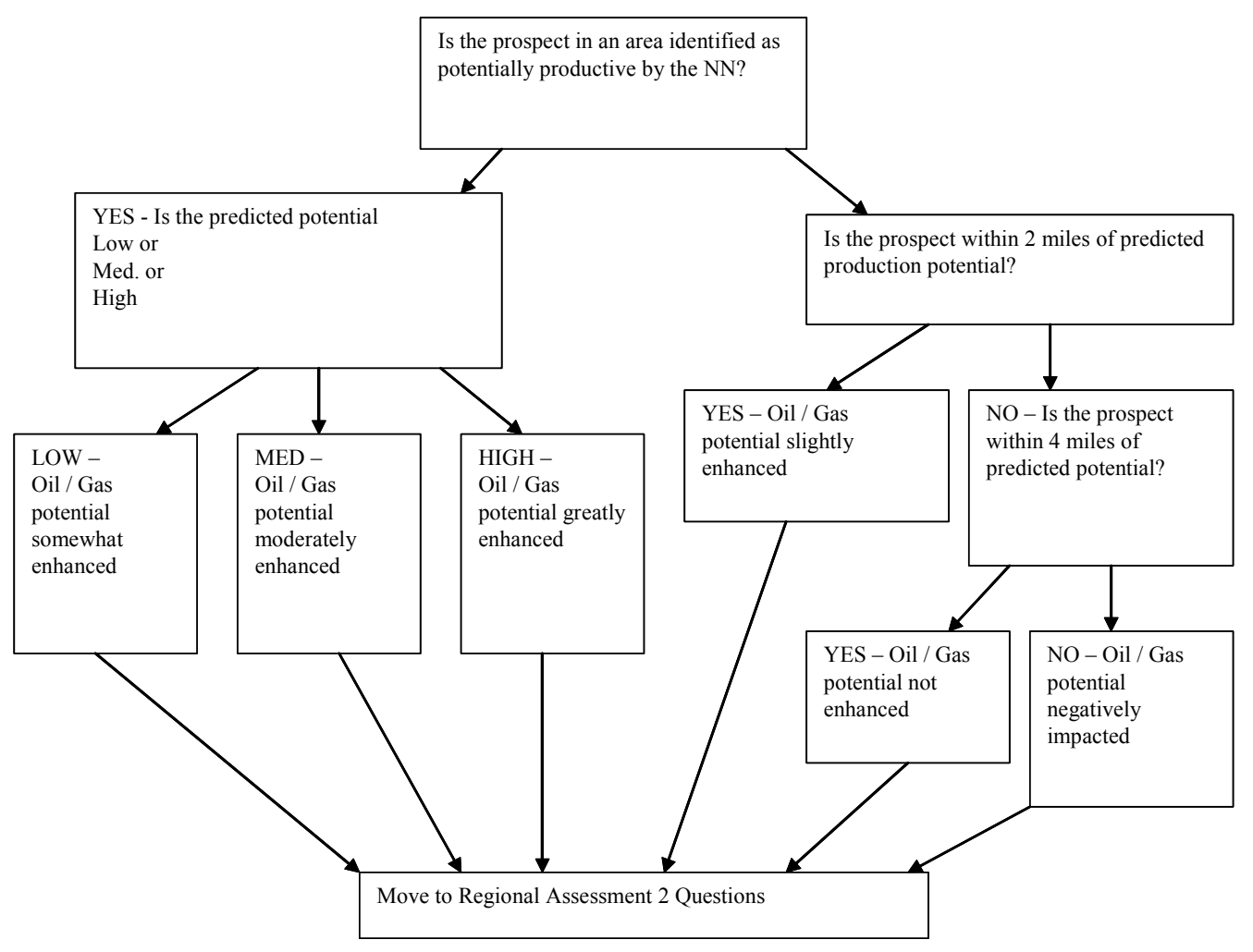

Fig. 21. Initial rules for regional assessment (Part I).

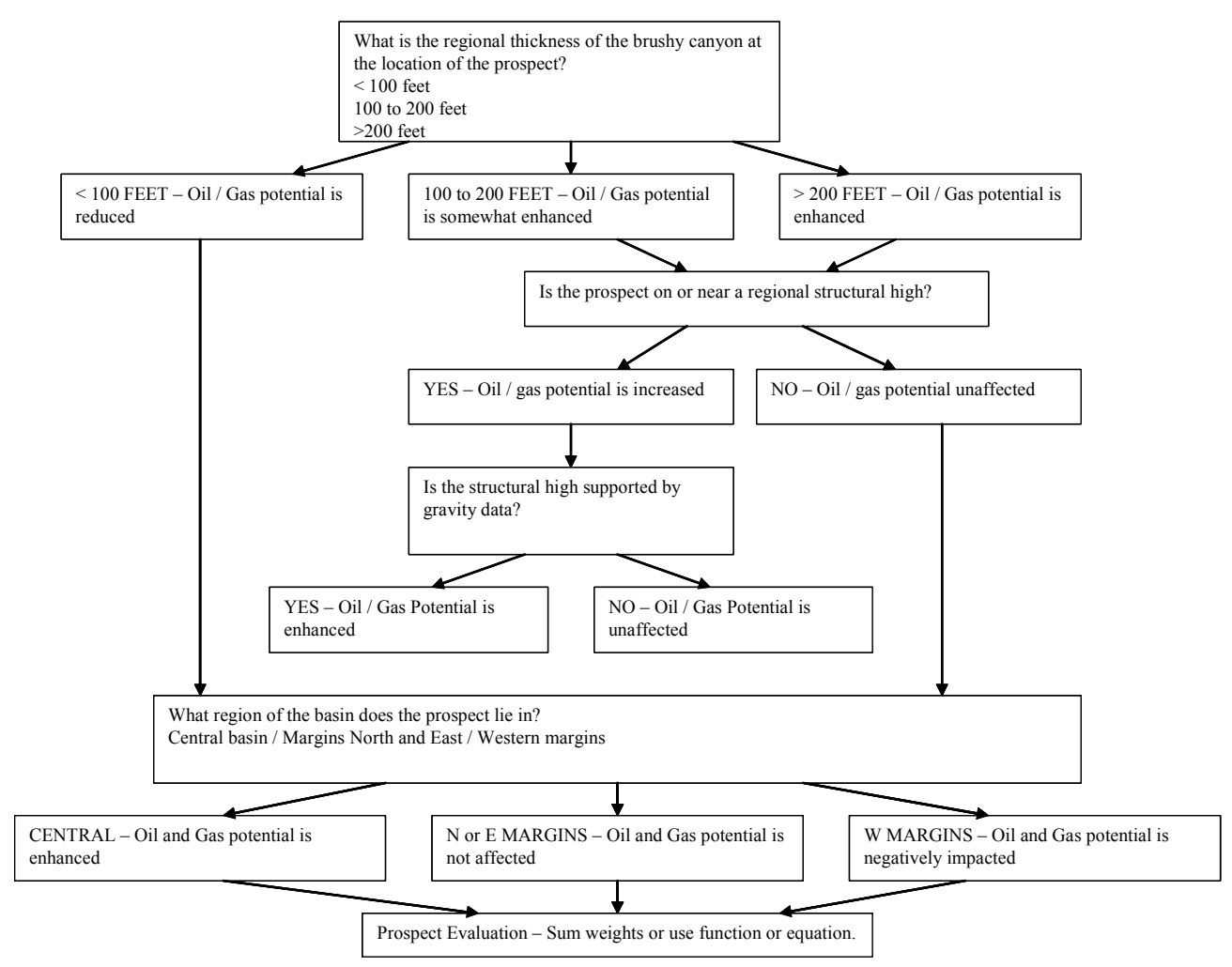

Fig. 22. Initial rules for regional assessment (Part II). 


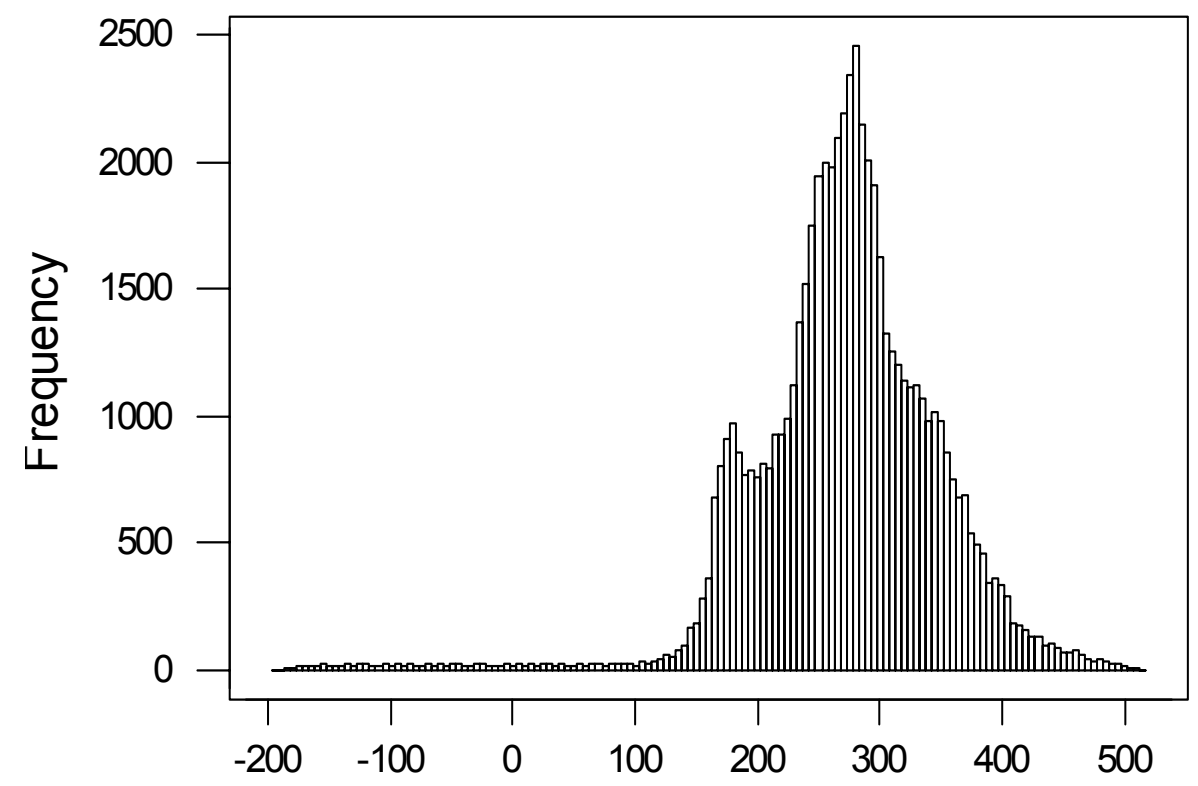

Fig. 23 Histogram showing the skewed nature and large mean of the thickness of the porous sand.

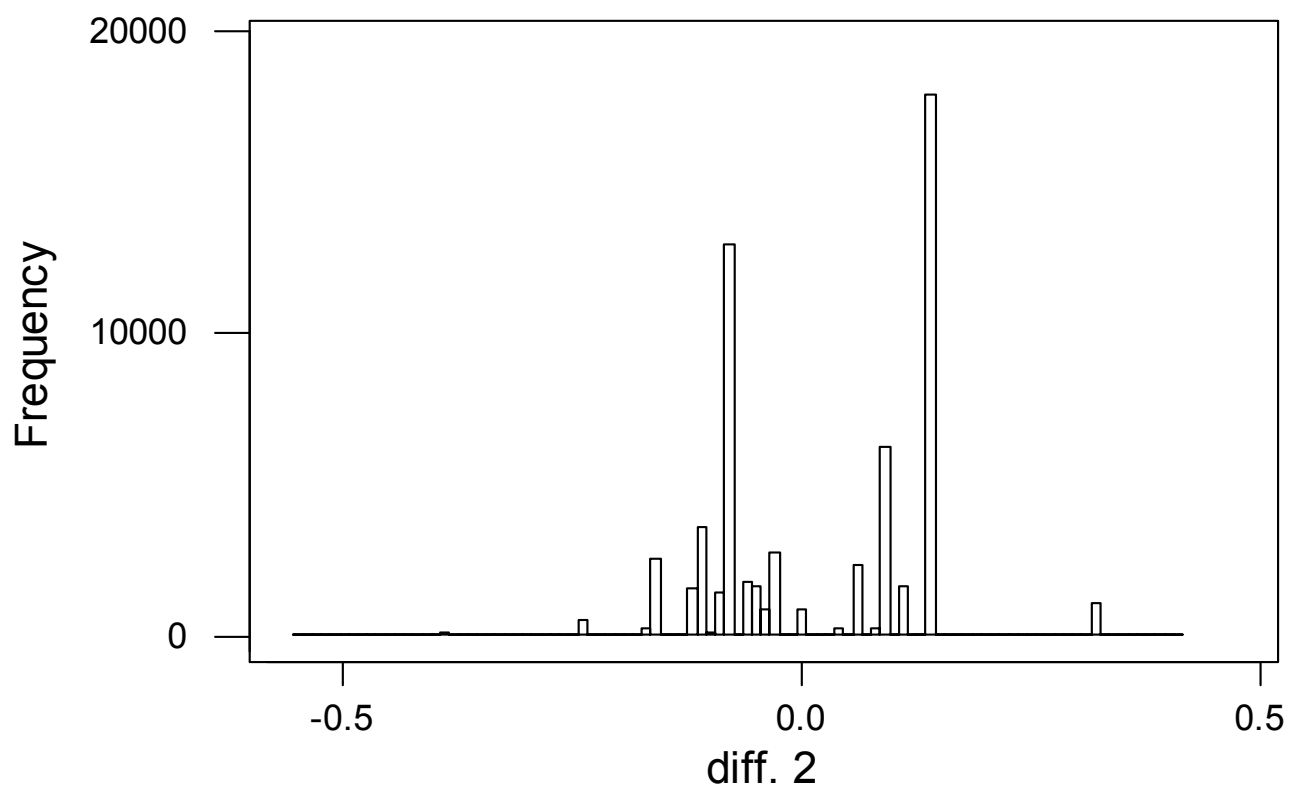

Fig. 24. Histogram of the differences in initial and enhanced estimate when thickness enhancement reduced. $($ Difference $=$ final - initial $)$ 


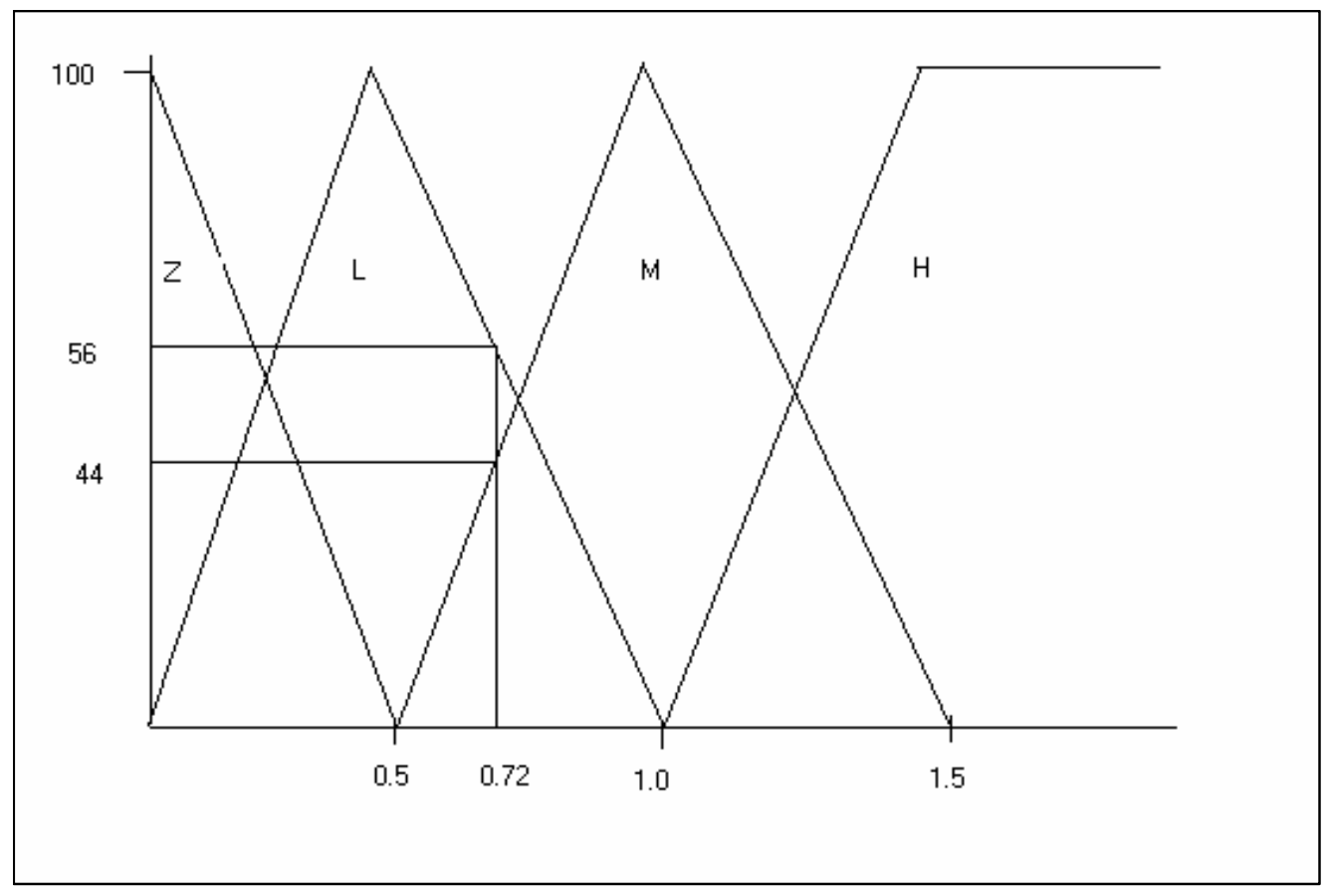

Fig. 25. Fuzzy membership functions for total organic carbon.

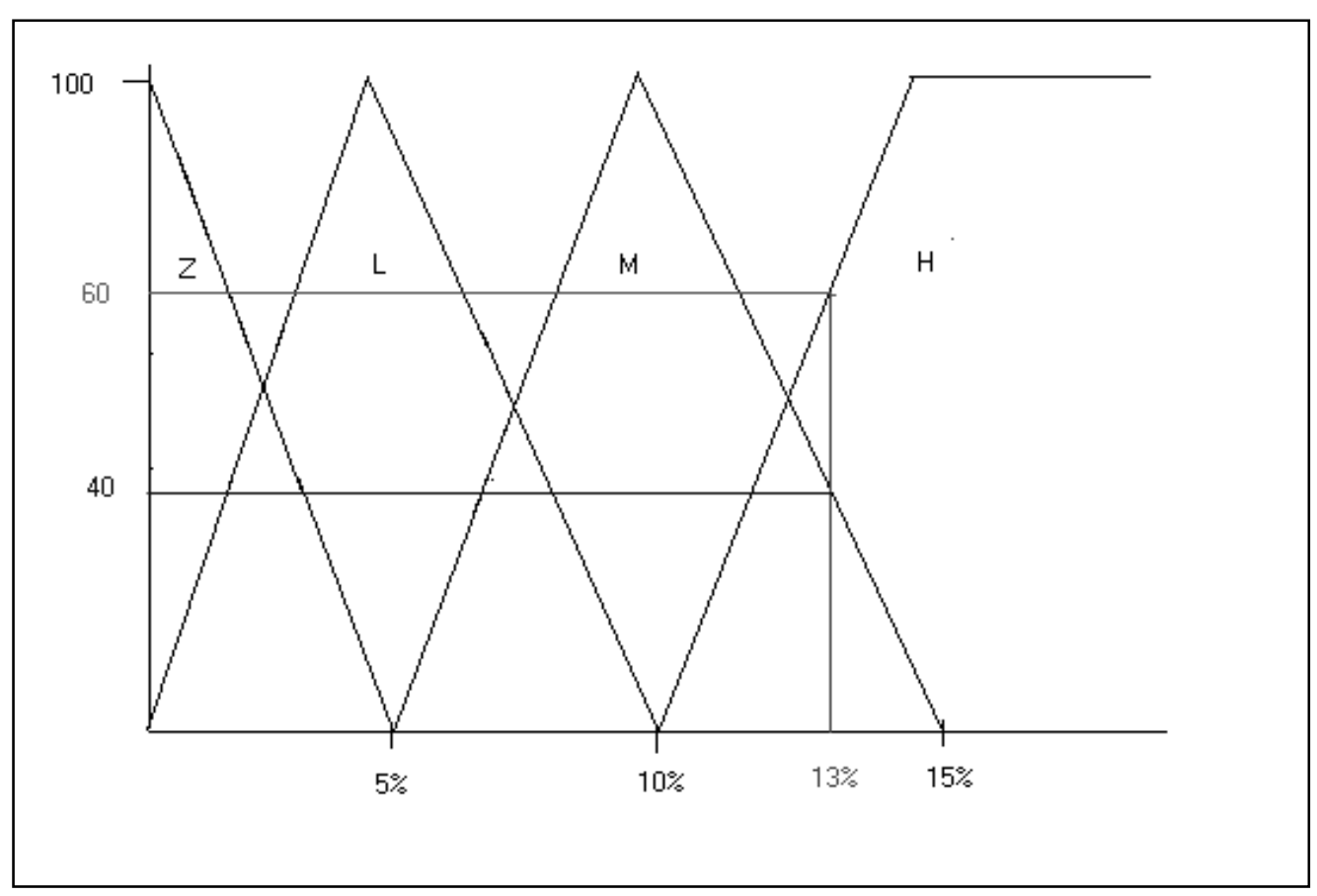

Fig. 26. Fuzzy membership functions for porosity. 


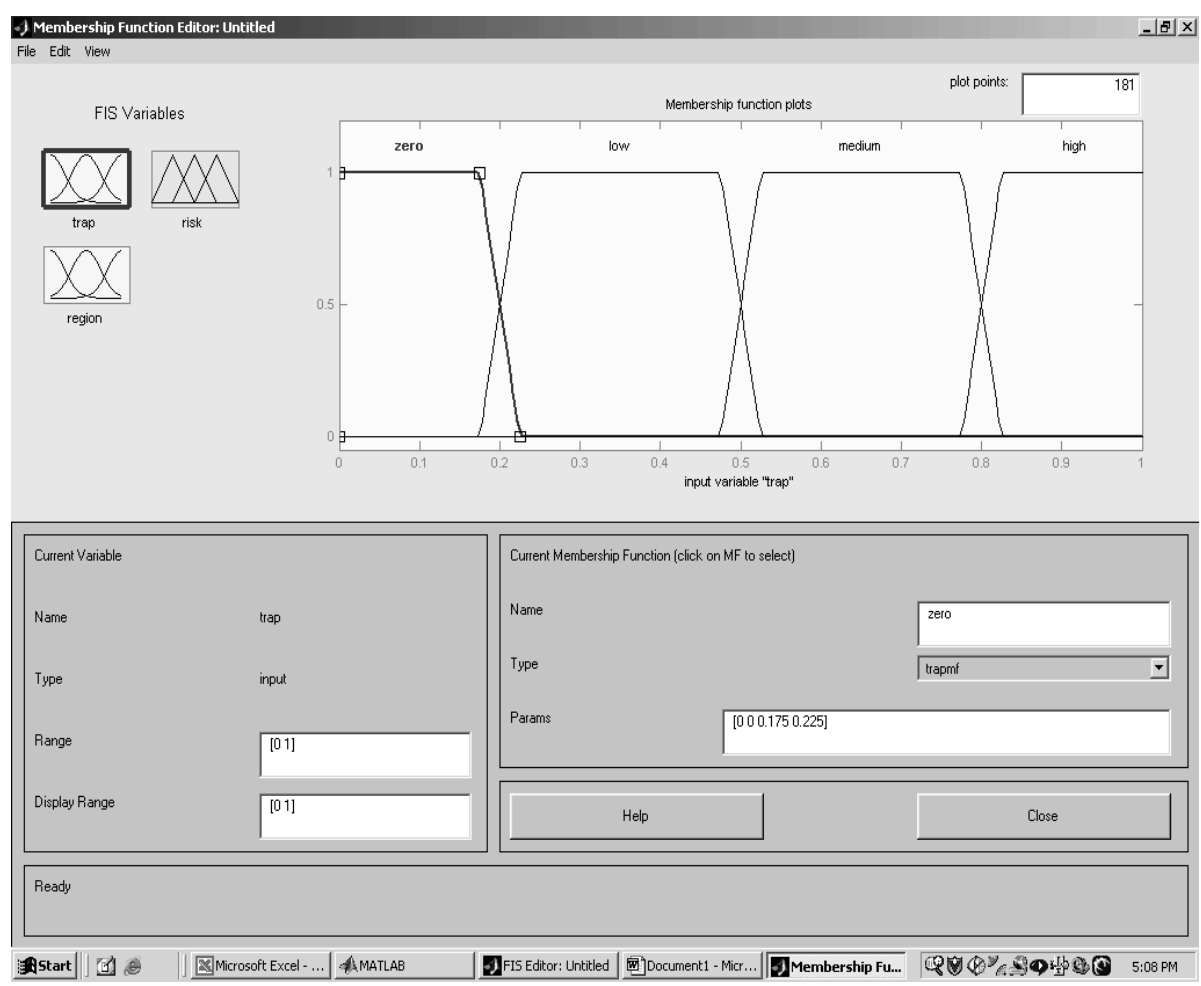

Fig. 27. Fuzzy membership curves for the trapmf function.

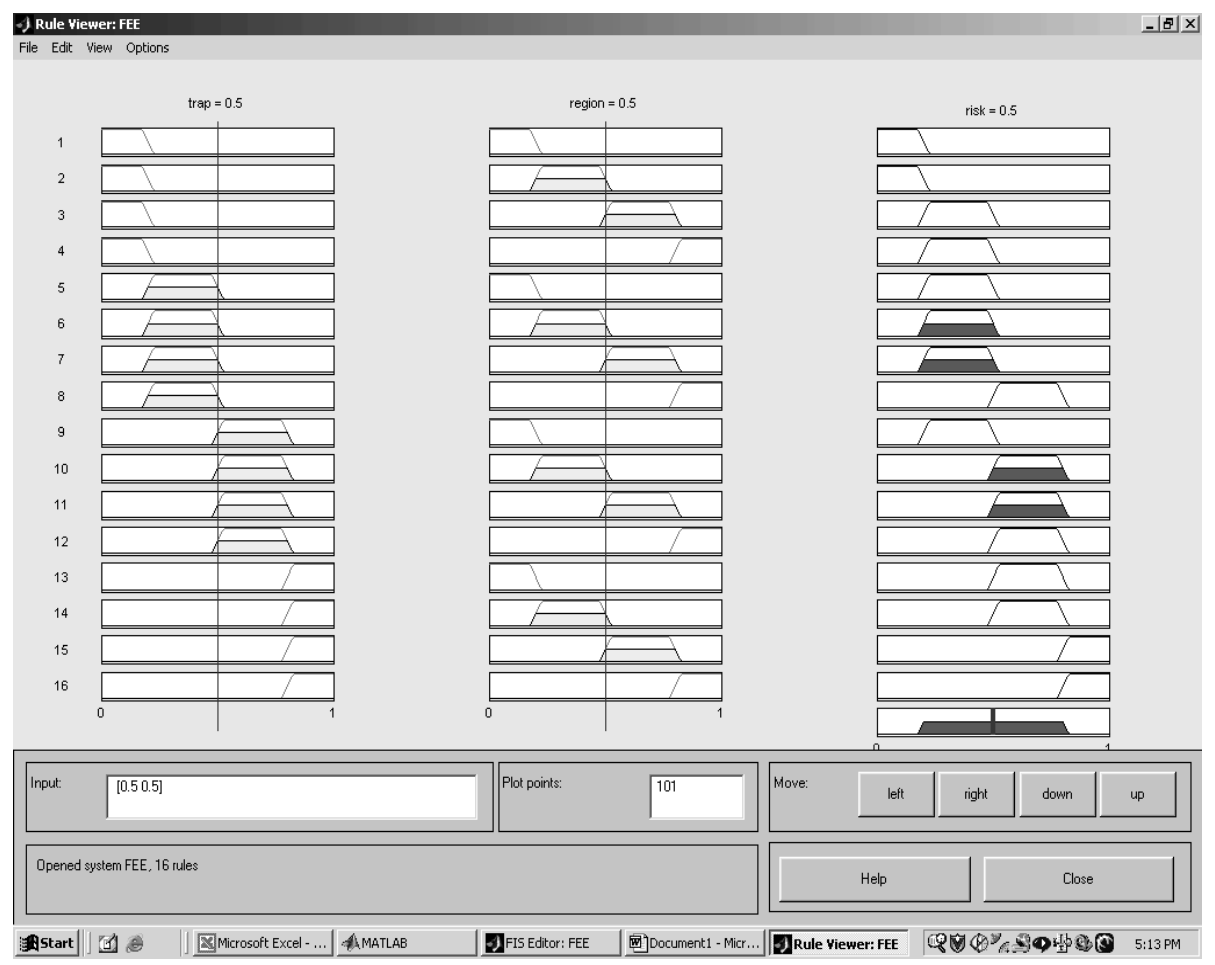

Fig. 28. Summary of all ranges for Trap (left), Regional (middle), and risk (right) as defined in Table 2. 


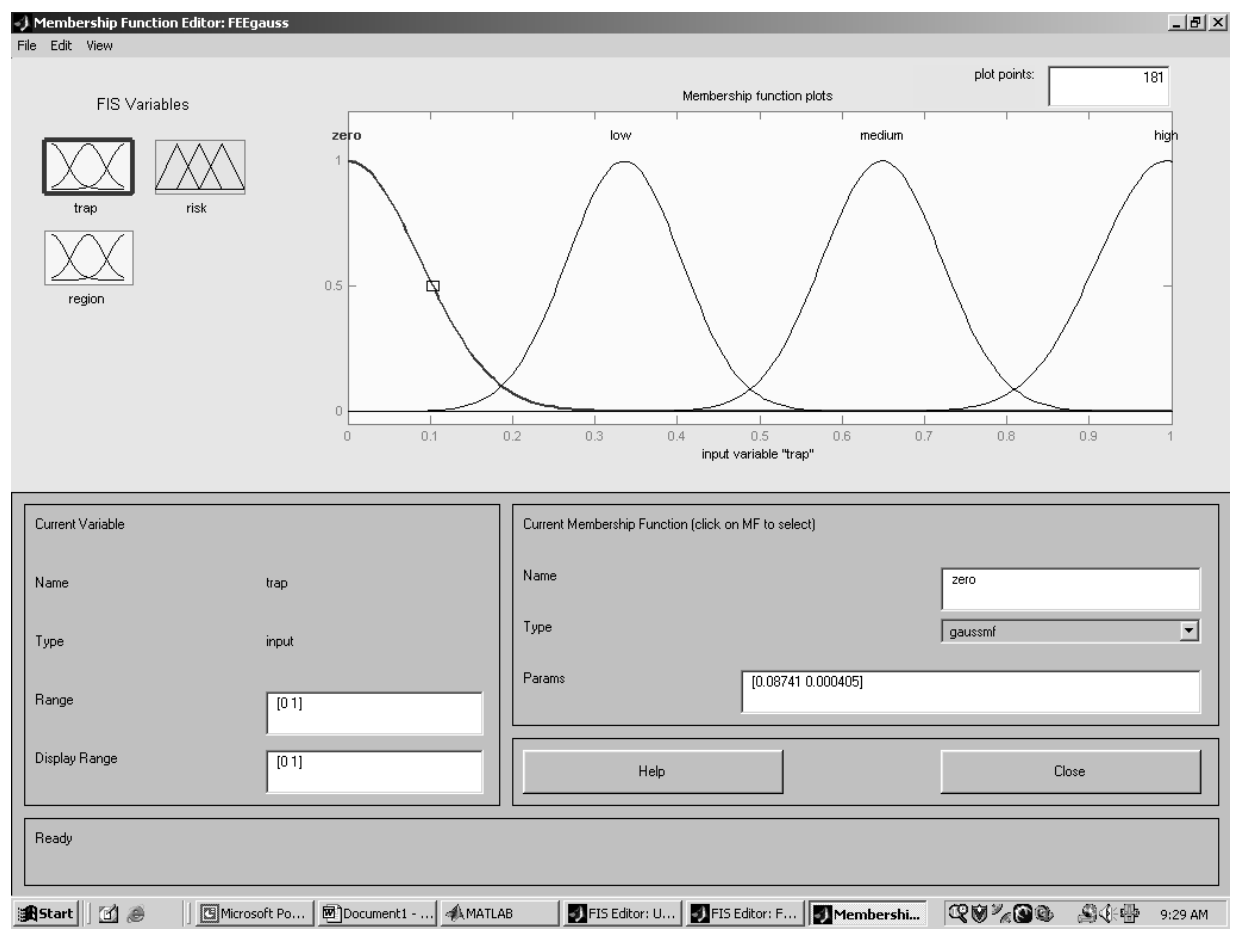

Fig. 29. Fuzzy membership curves for the gaussmf function.

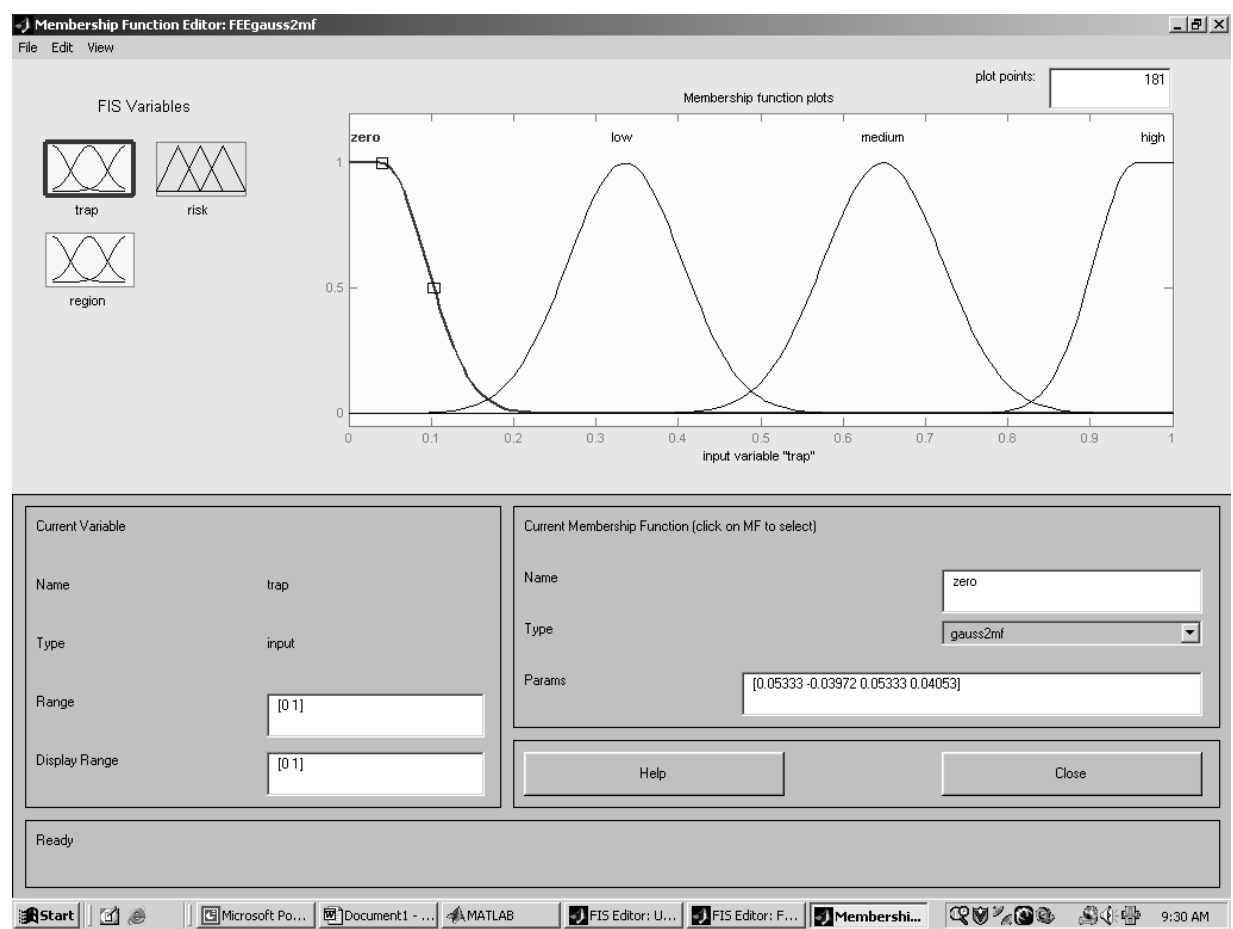

Fig. 30. Fuzzy membership curves for the gauss $2 \mathrm{mf}$ function. 


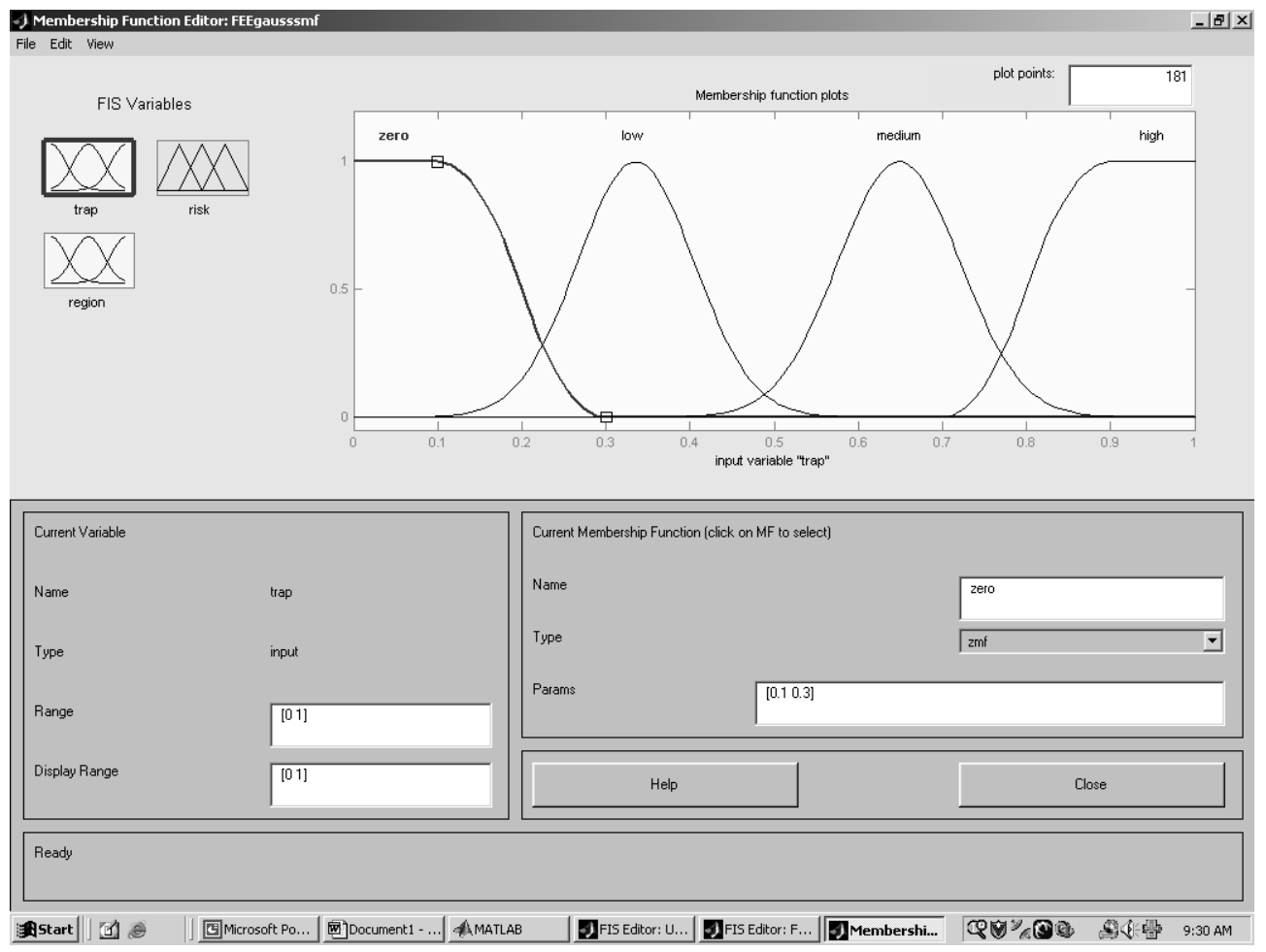

Fig. 31. Fuzzy membership curves for a composite of zmf and smf functions.

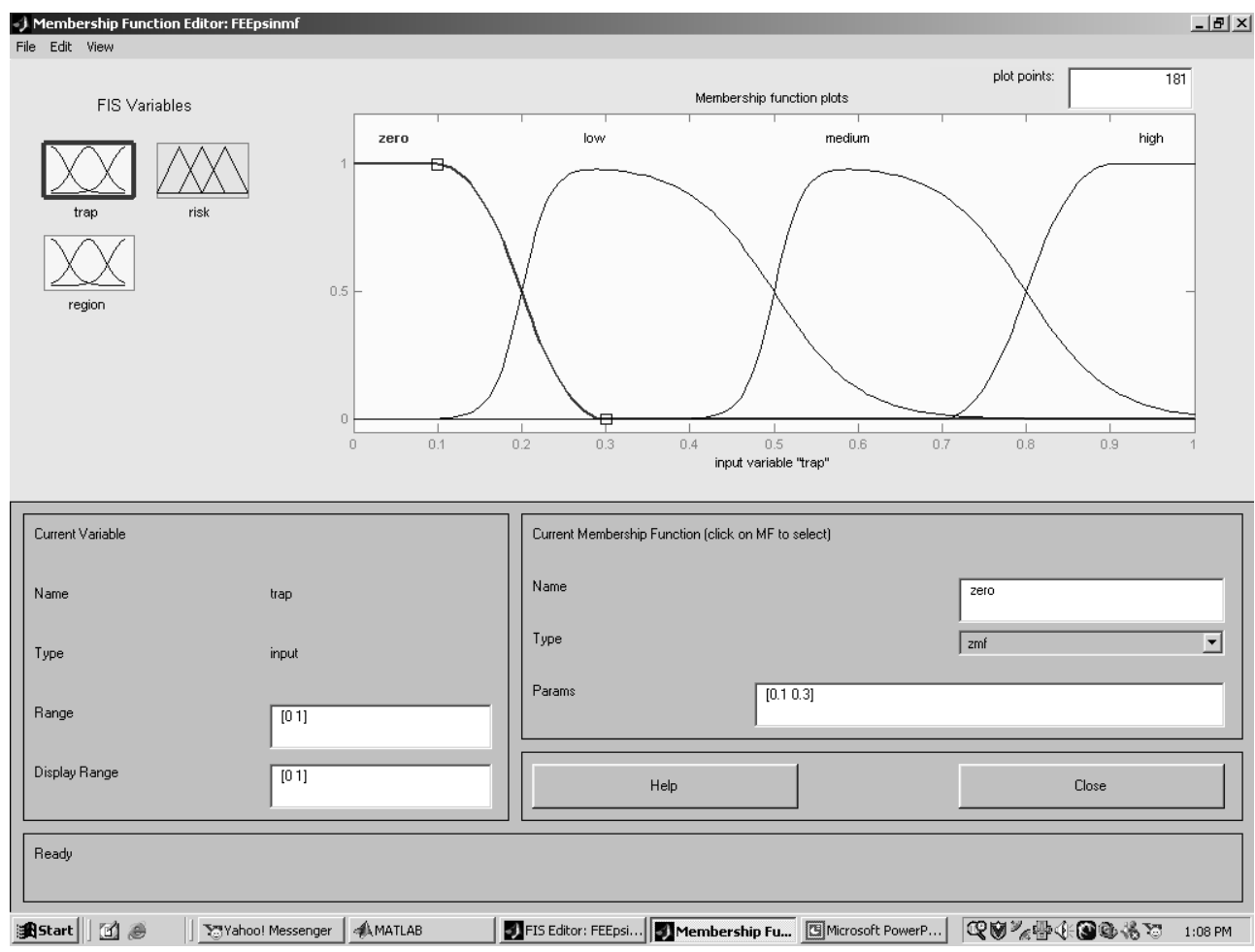

Fig. 32. Plot of membership curves with "dsinmf" and "psinmf" used for both low and medium and the "zmf" and "smf" functions for the zero and high. 


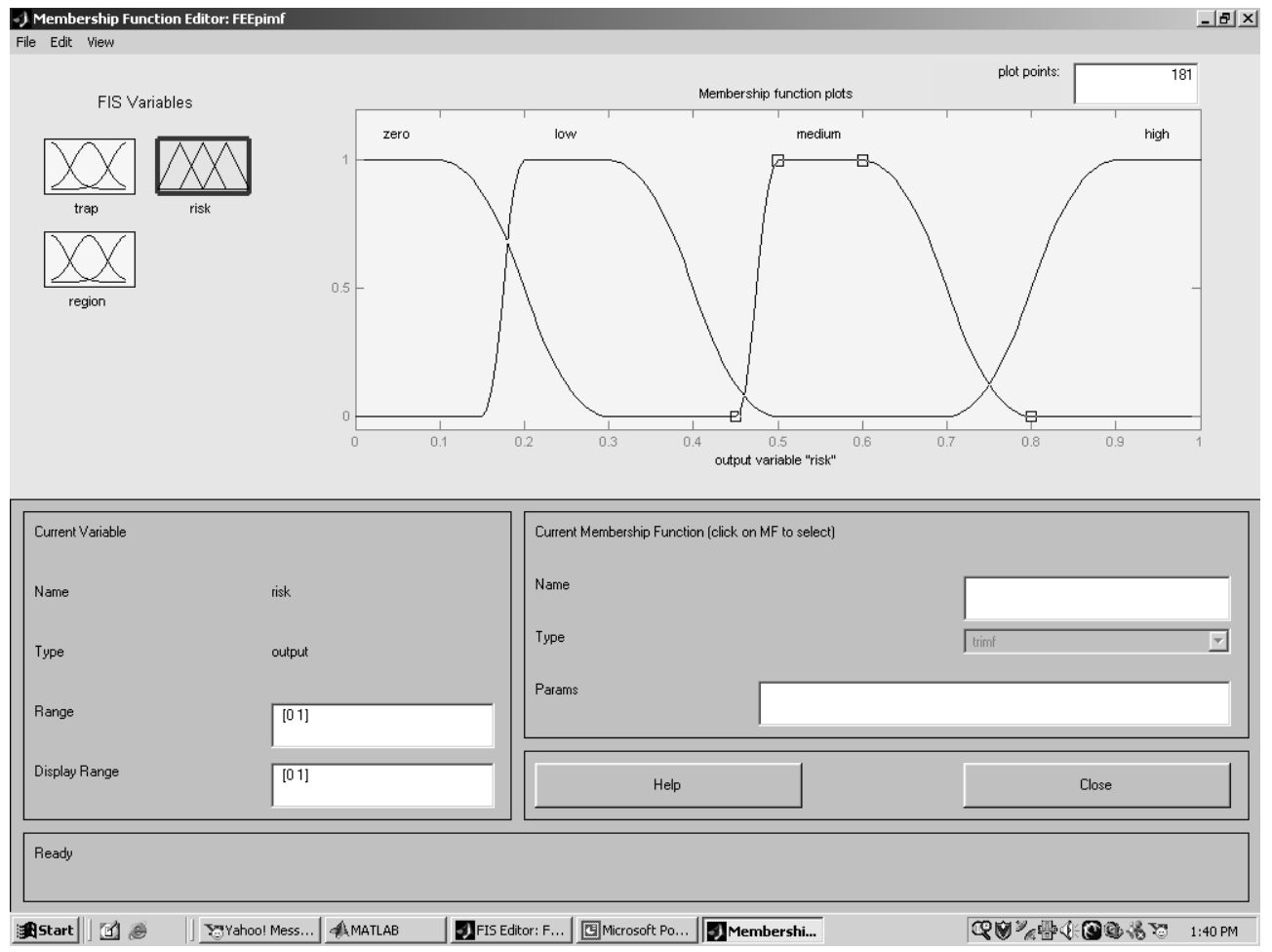

Fig. 33. Plot of membership curves when the "pimf" function is used for low and medium parameters and the "zmf" and "smf" functions for zero and high

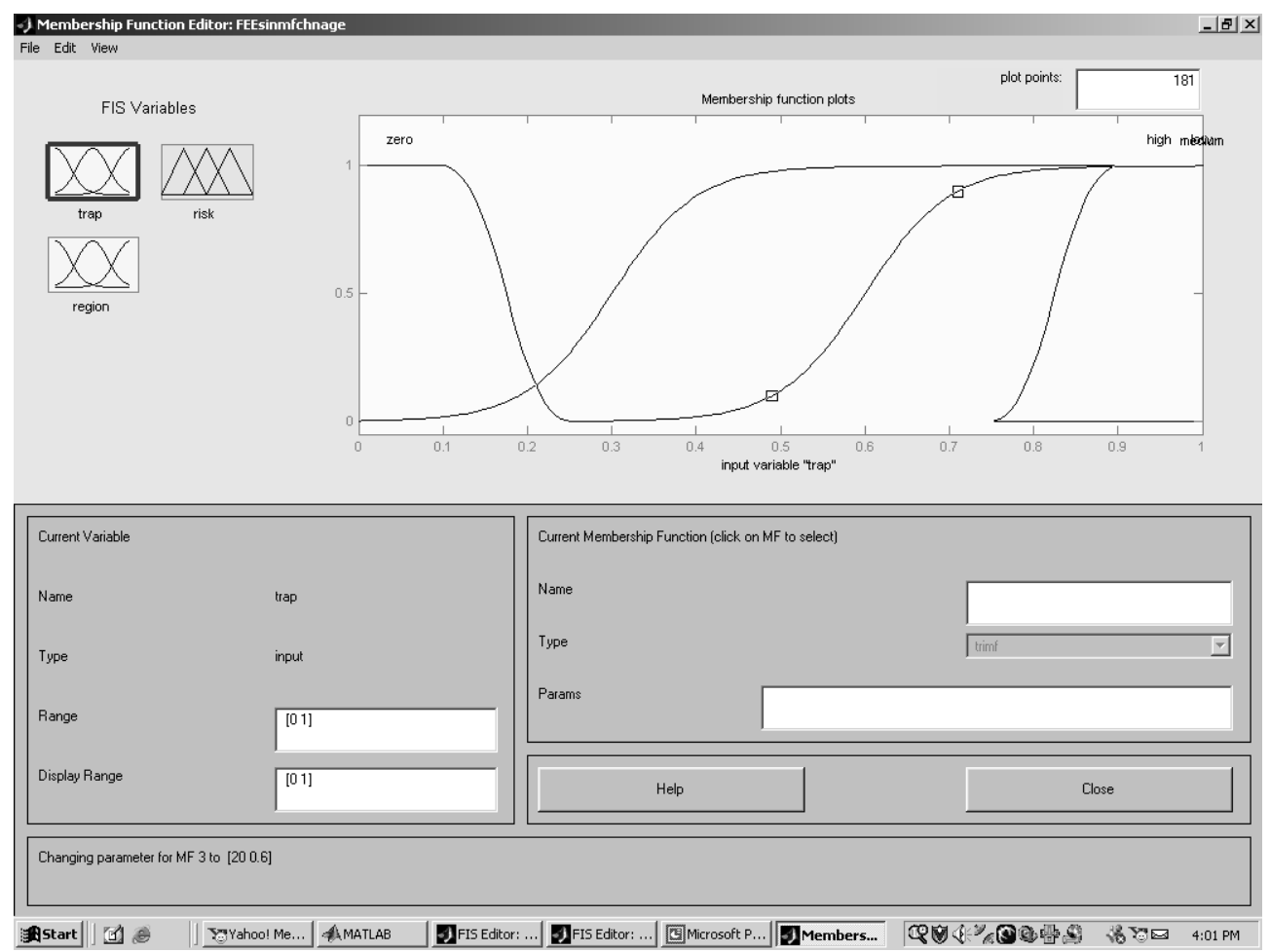

Fig. 34. Plot of membership curves using "sin" curves for Low and Medium and using "zmf" and "smf" curves for the zero and high parameters. 


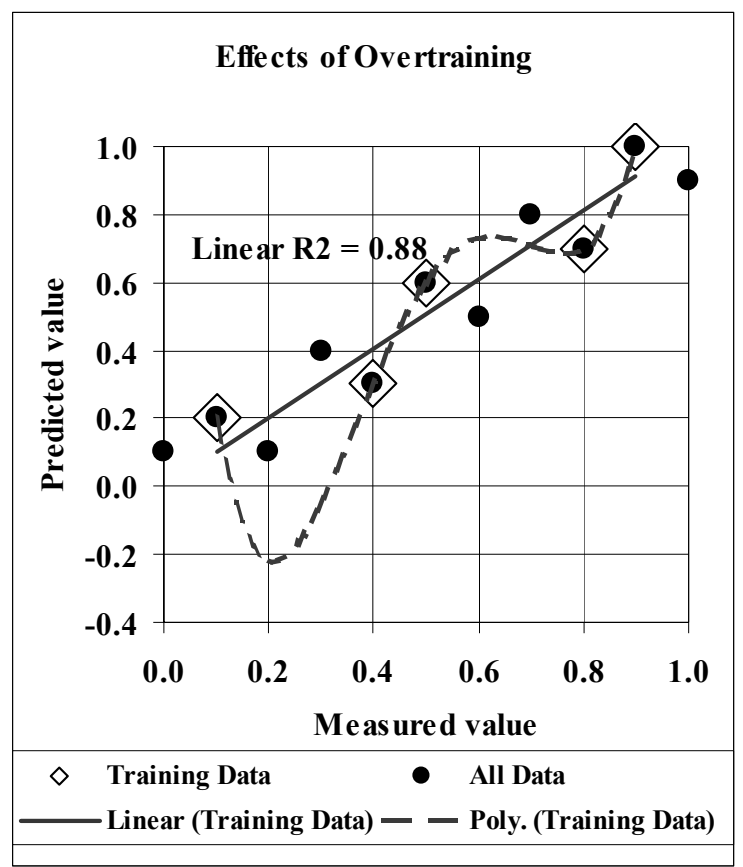

Fig. 35. Overtraining cartoon demonstrates that large errors are possible with $100 \%$ correlation coefficient.

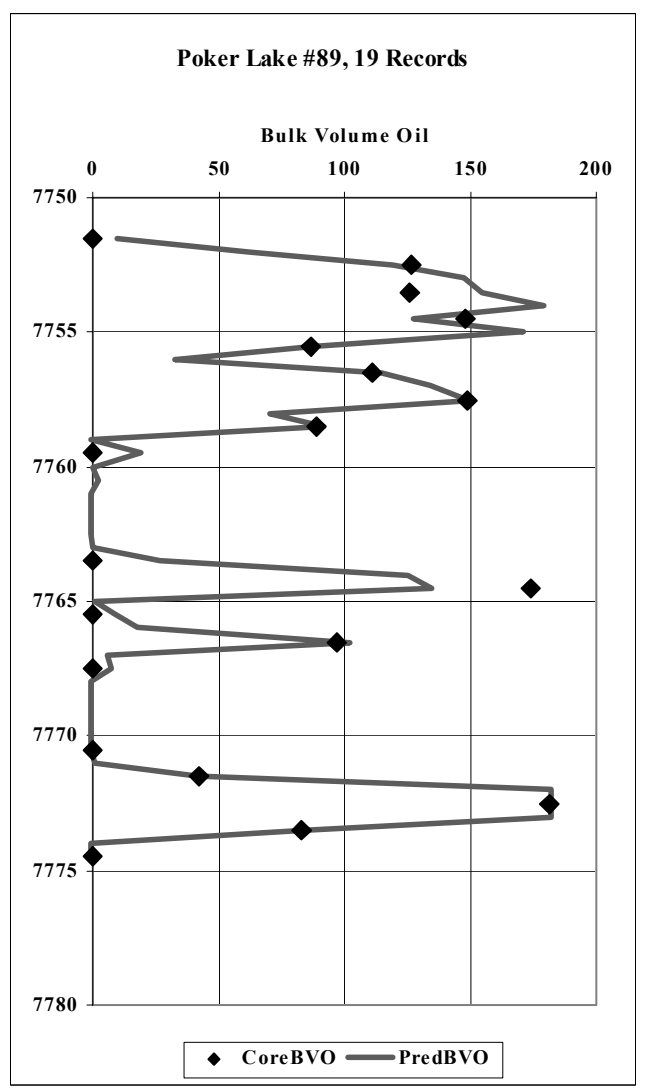

Fig. 36. Small core dataset trains to almost $100 \%$ correlation coefficient. 


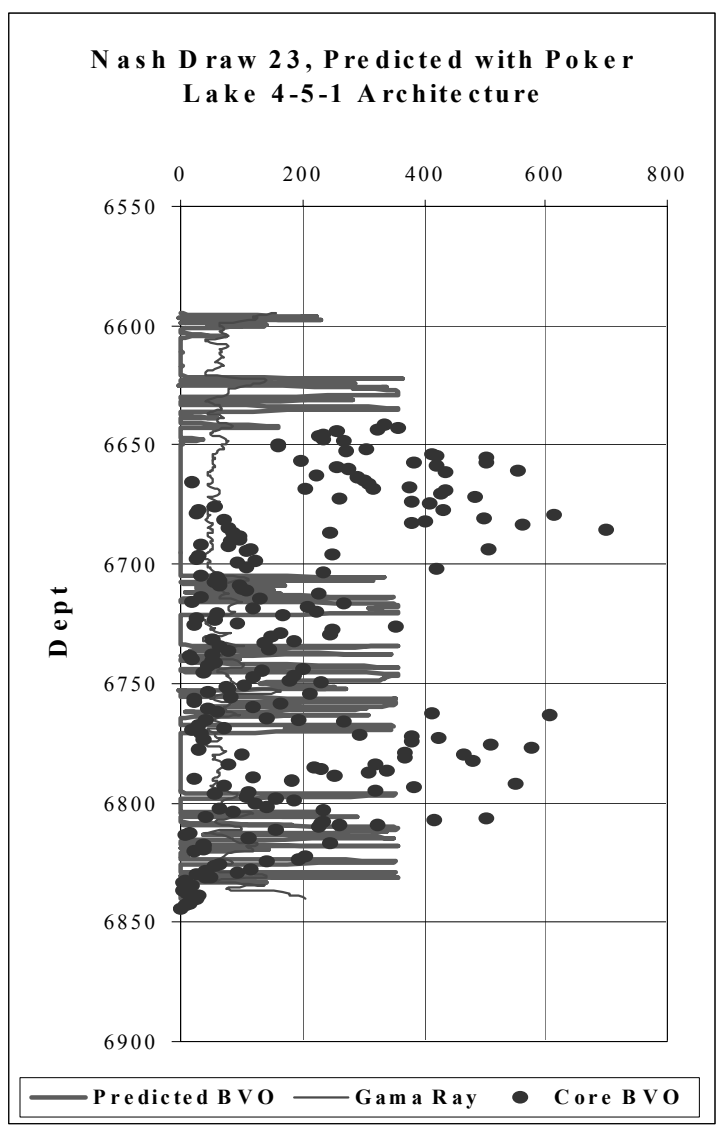

Fig. 37. Training to almost $100 \%$ correlation coefficient produces an extremely poor blind test.

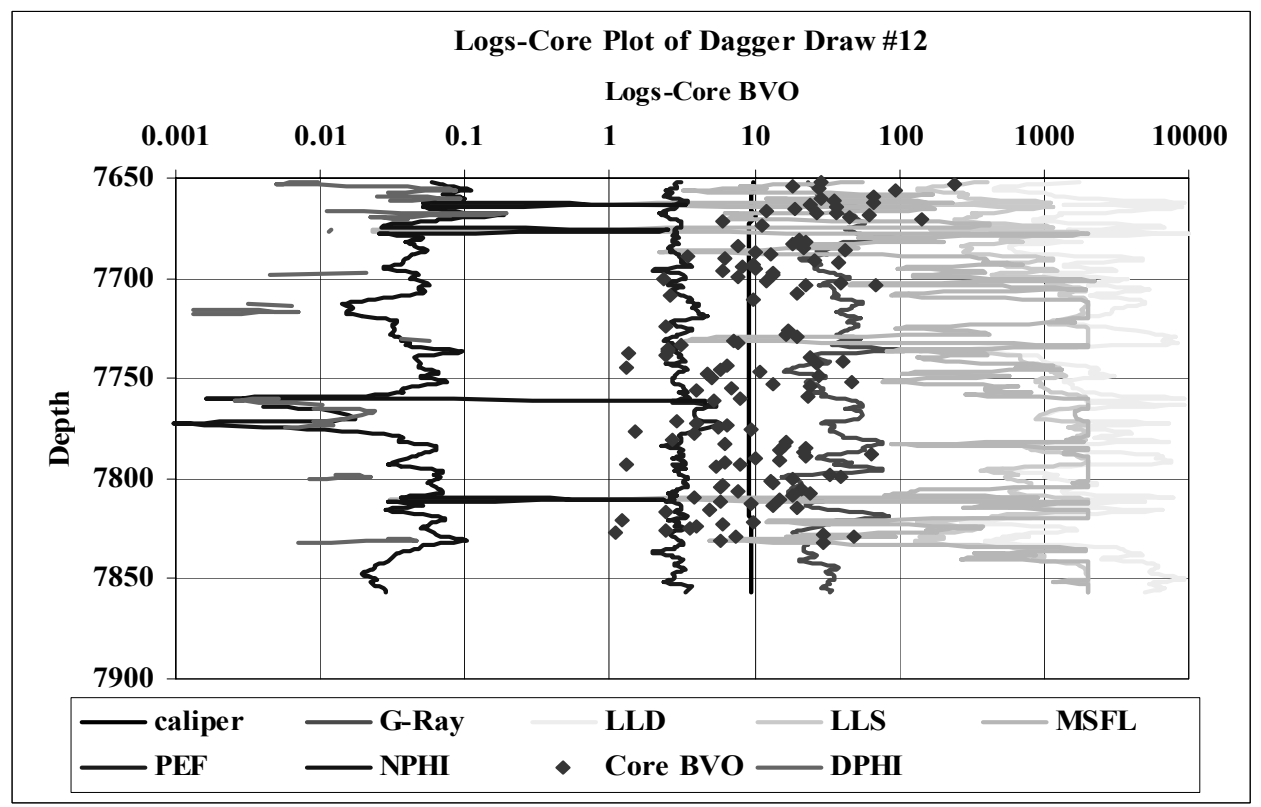

Fig. 38. Typical logs and core measurements through $200 \mathrm{ft}$ of an algal mound reservoir. 


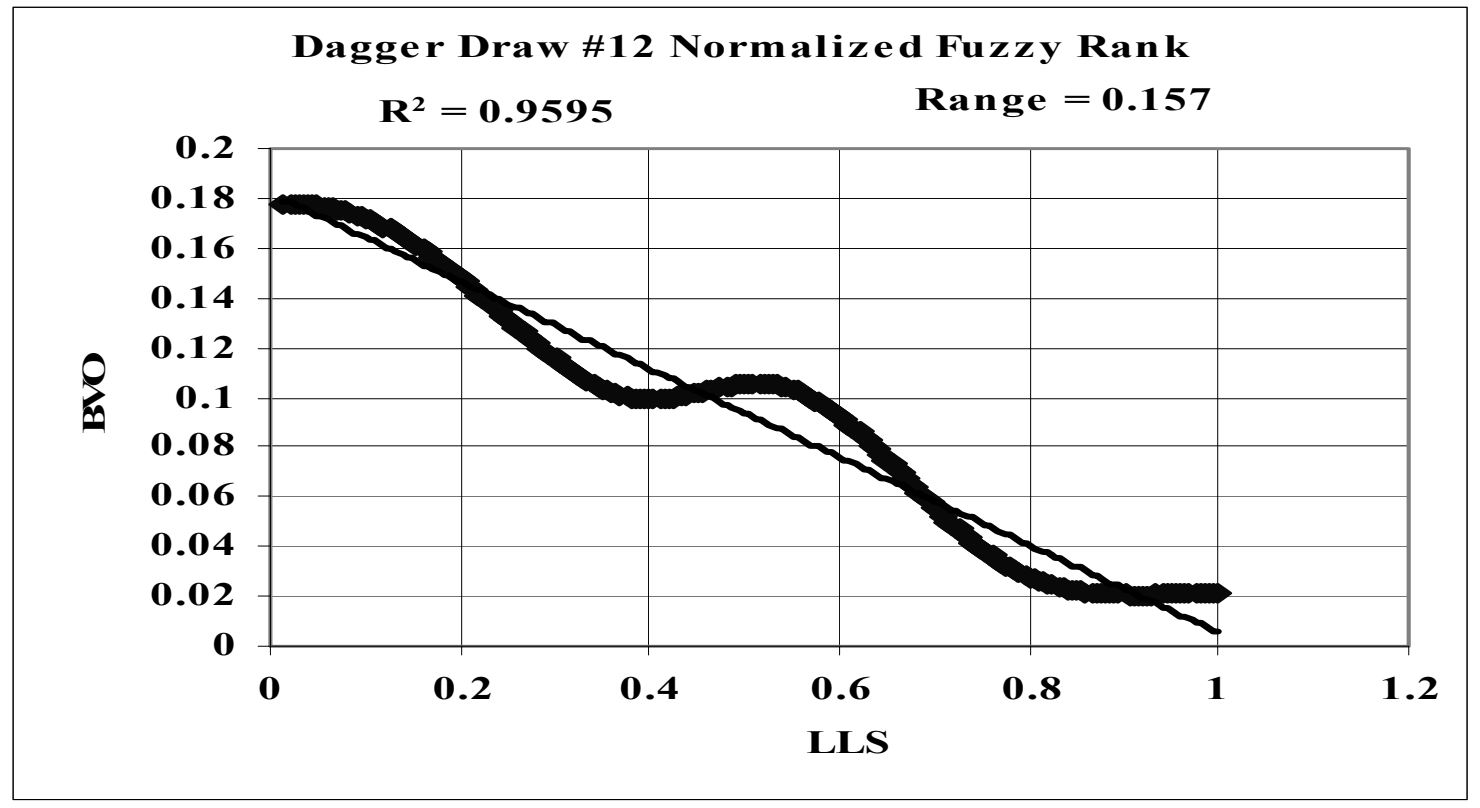

Fig. 39. Shallow resistivity log fuzzy curve.

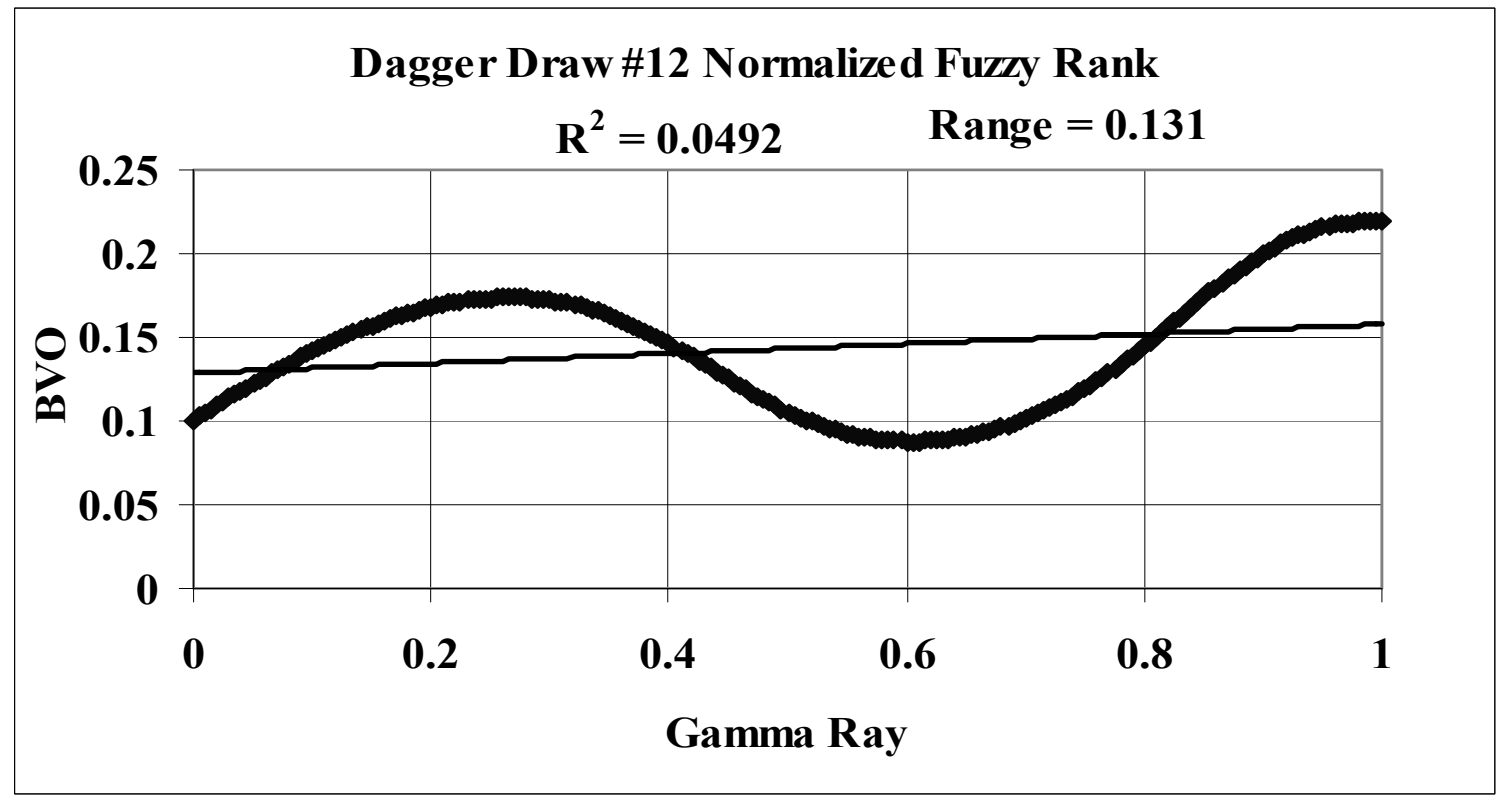

Fig. 40. Gamma ray log fuzzy curve. 


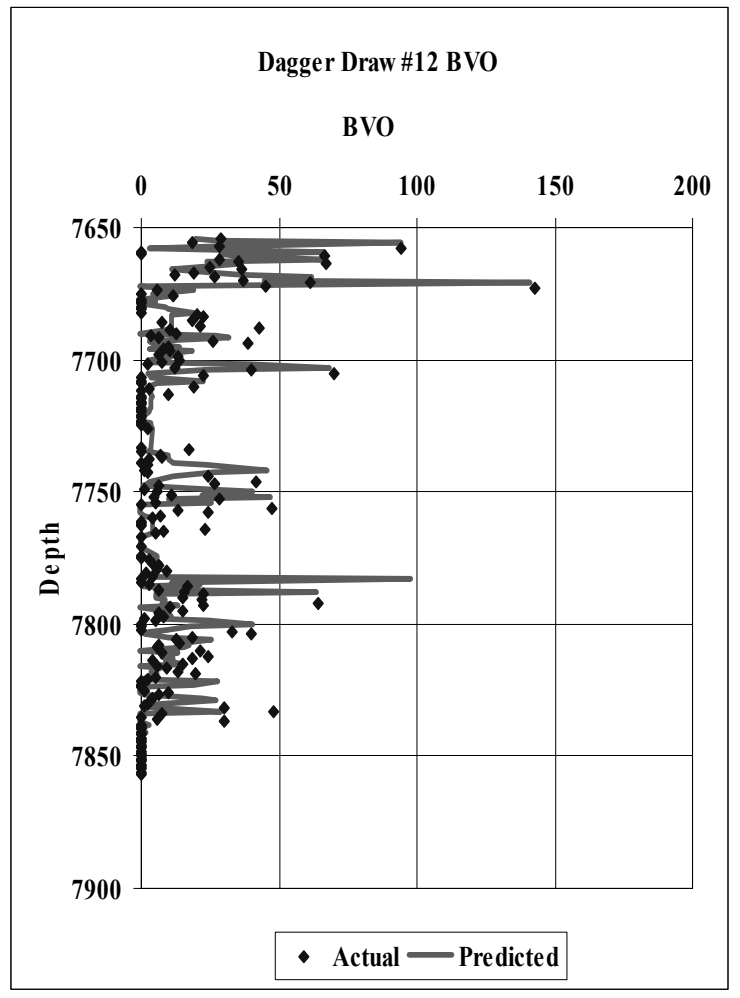

Fig. 41. Trained neural network BVO; pseudo-log vs. core measurements.

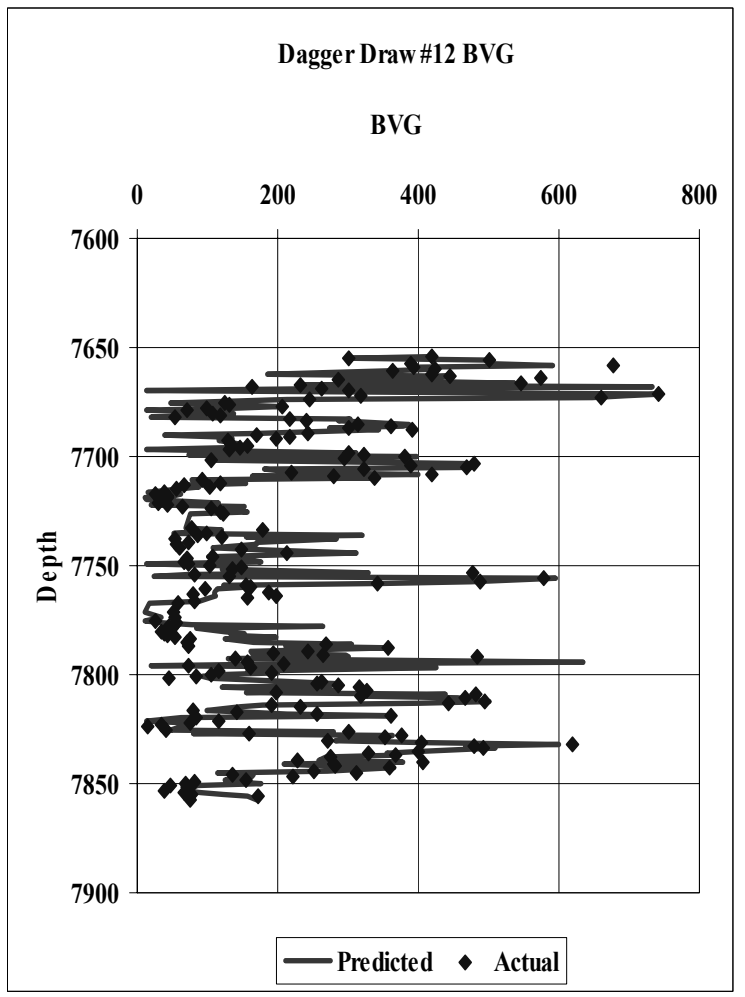

Fig. 42. Trained neural network BVG; pseudo-log vs. core measurements. 


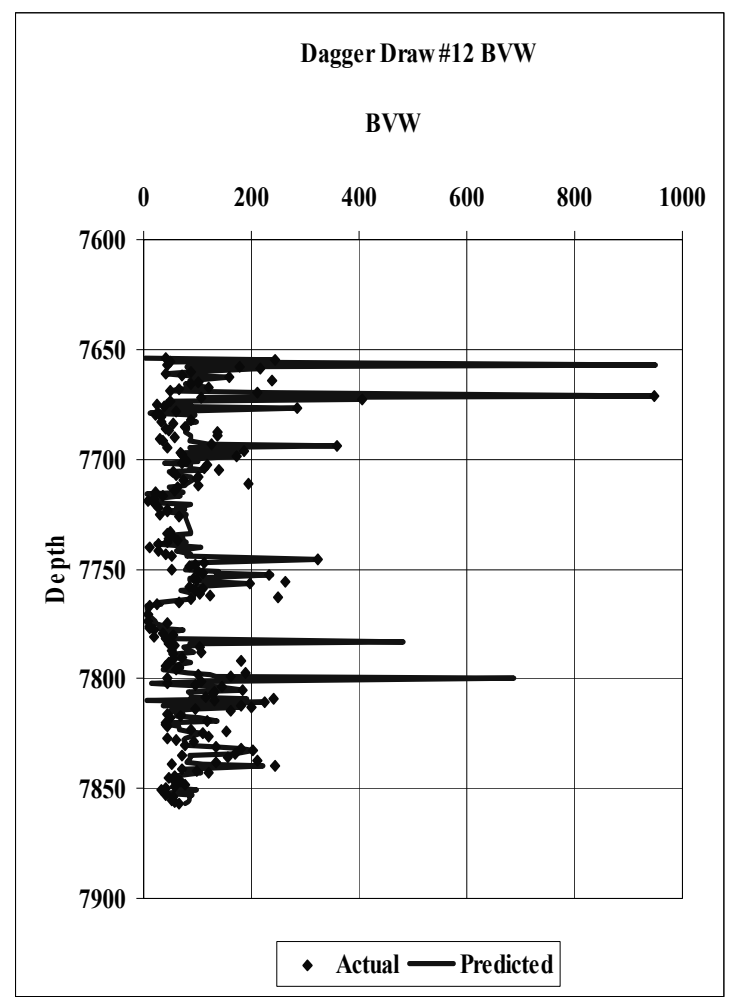

Fig. 43. Trained neural network BVW; pseudo-log vs. core measurements.

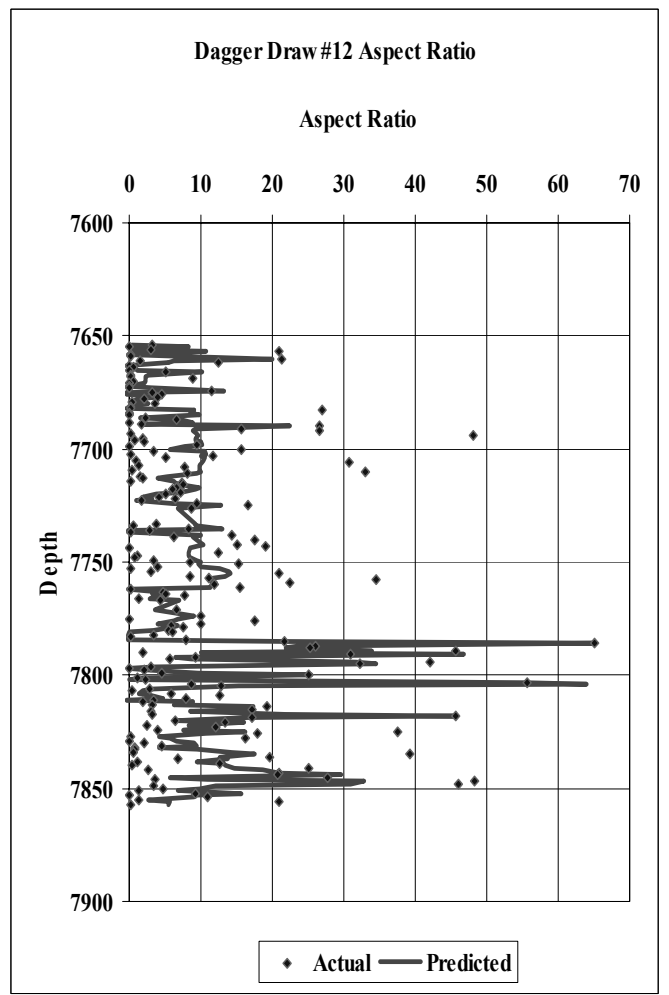

Fig. 44. Trained neural network aspect ratio; pseudo-log vs. core measurements. 


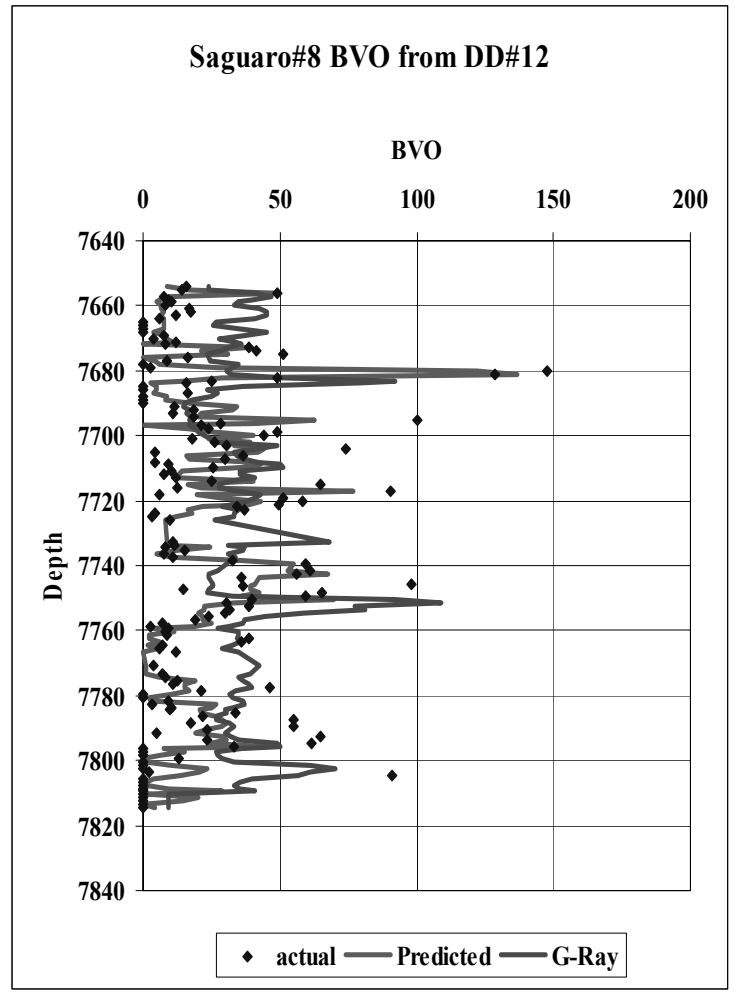

Fig. 45. Blind test of DD\# 12 BVO neural network.

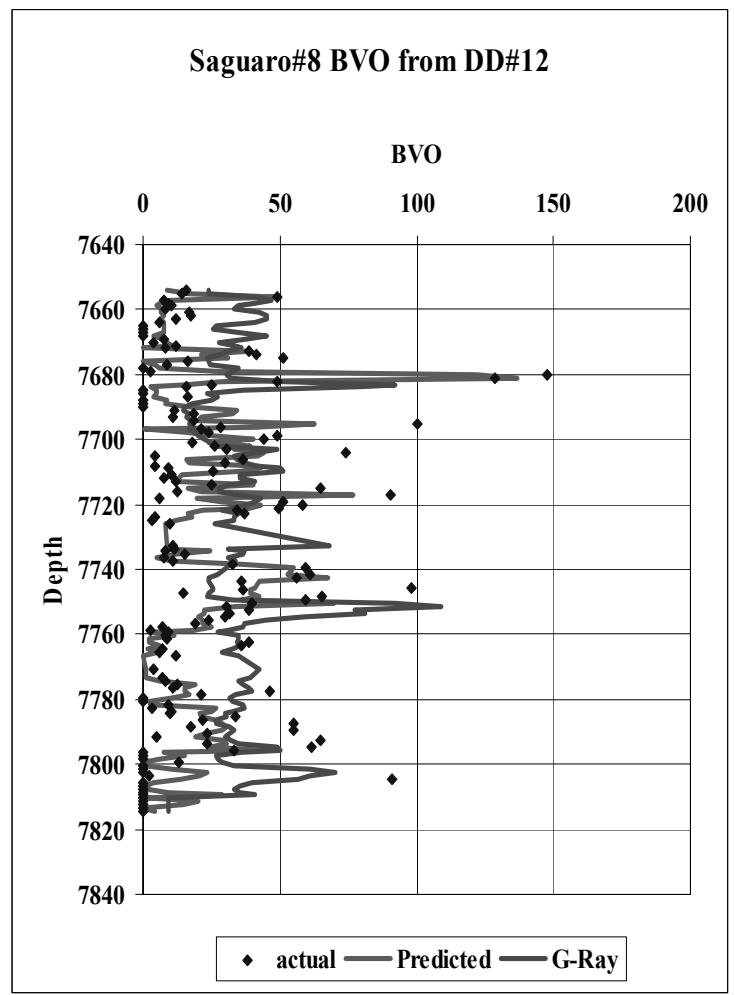

Fig. 46. Blind test of DD\# 12 BVG neural network. 


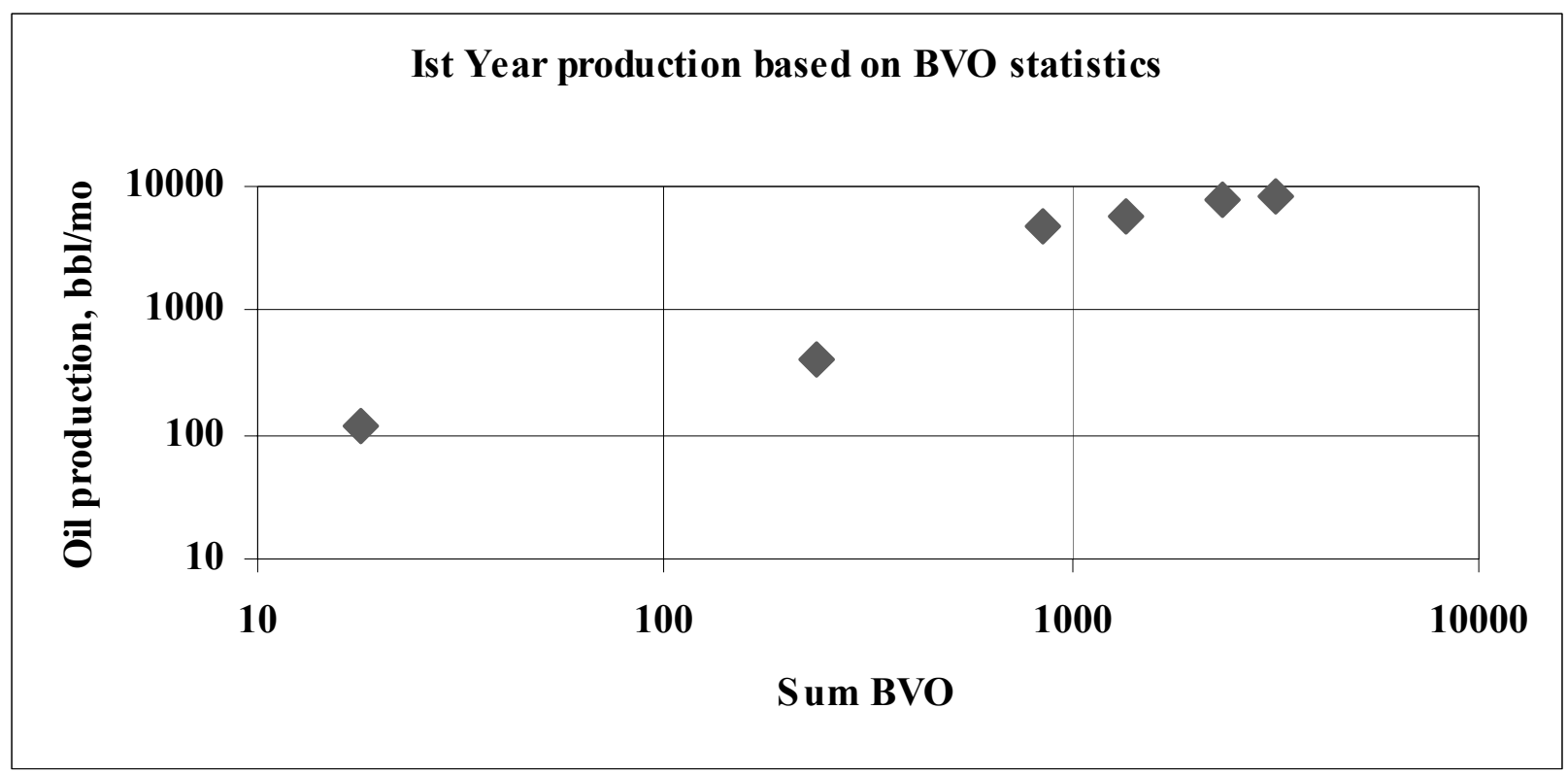

Fig. 47. Sum of pseudo-log BVO; log predictions vs. initial oil rate.

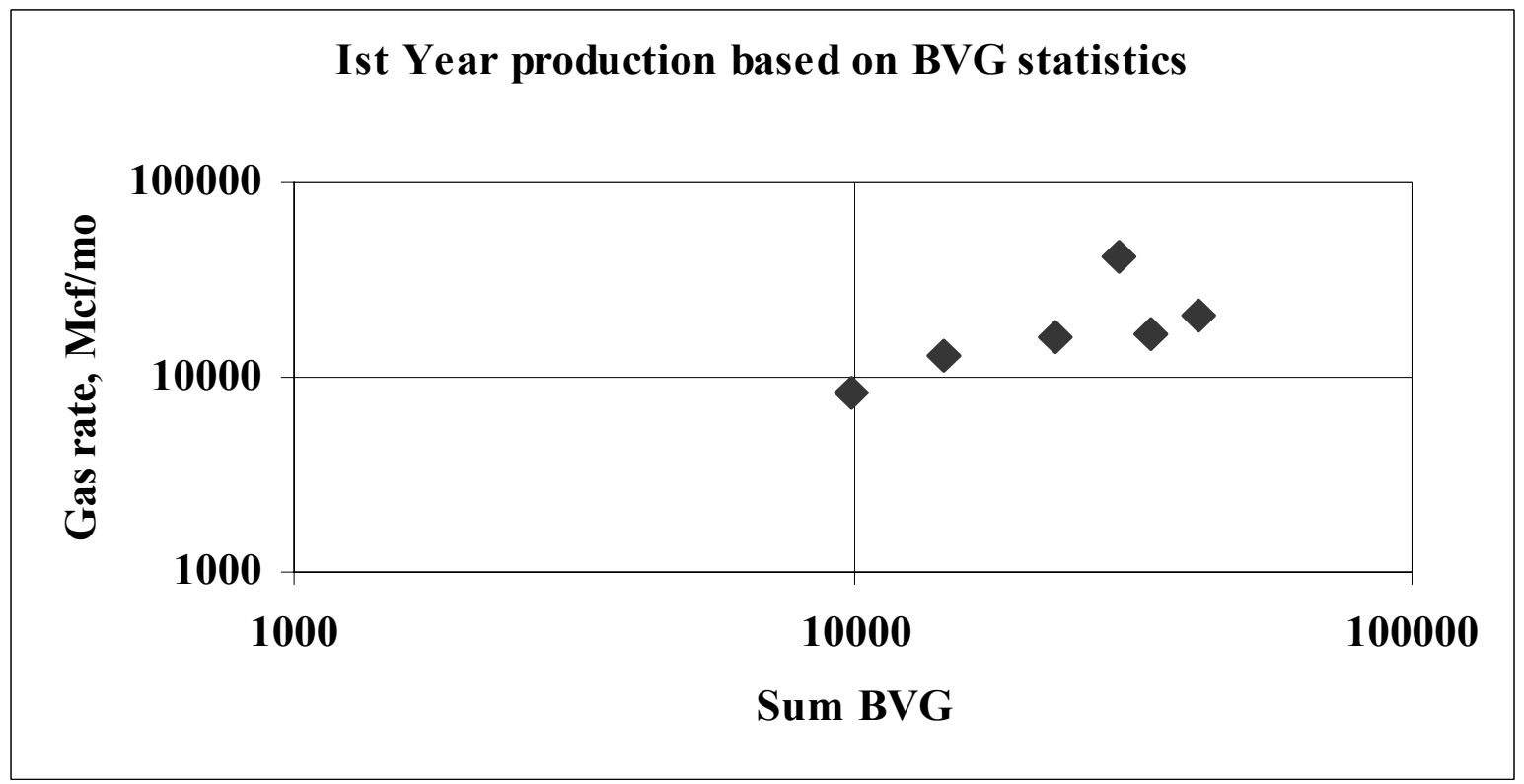

Fig. 48. Sum of pseudo-log BVG; log predictions vs. initial gas rate. 


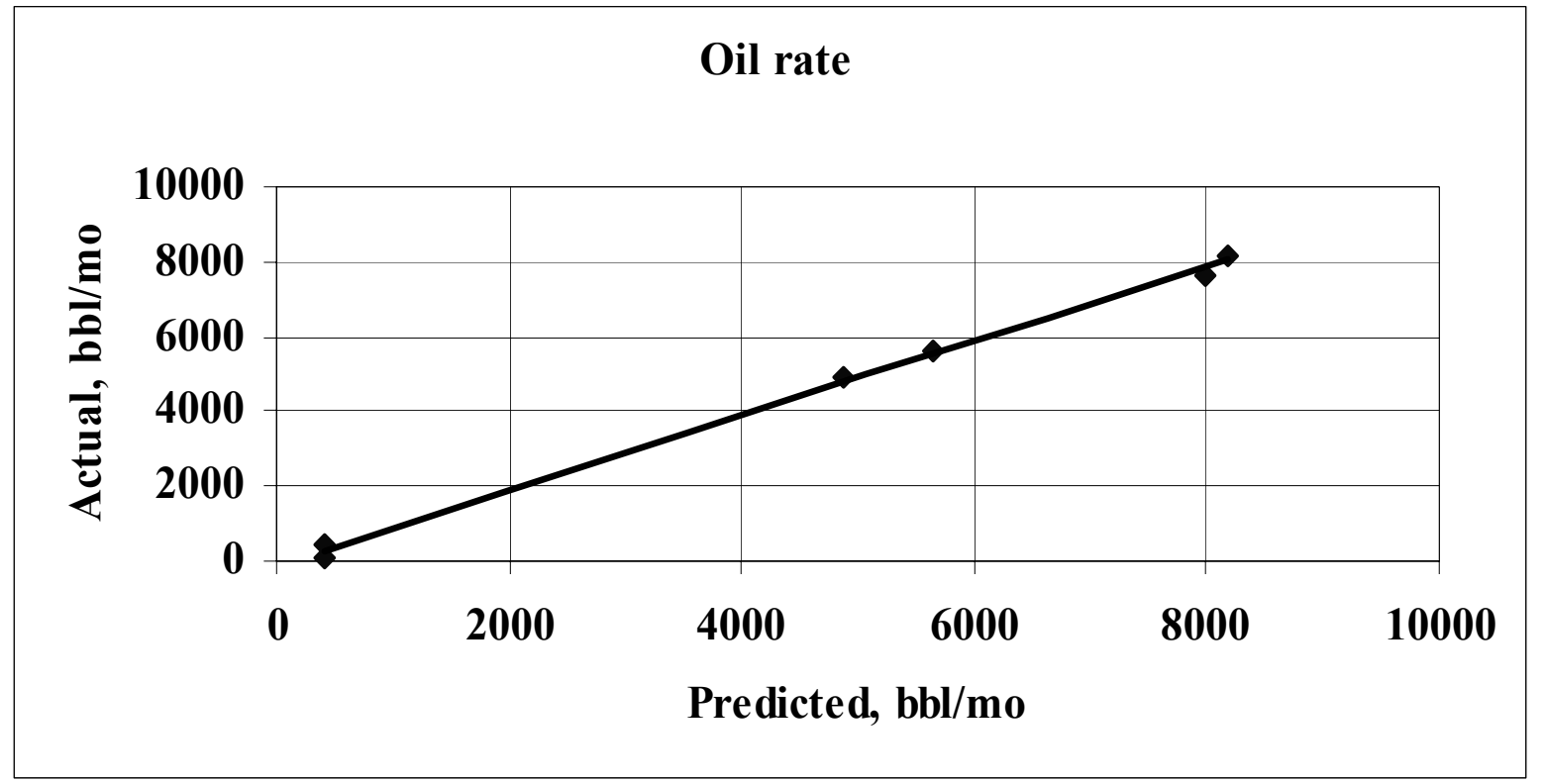

Fig. 49. Sum and average neural network predictions vs. actual initial oil rate.

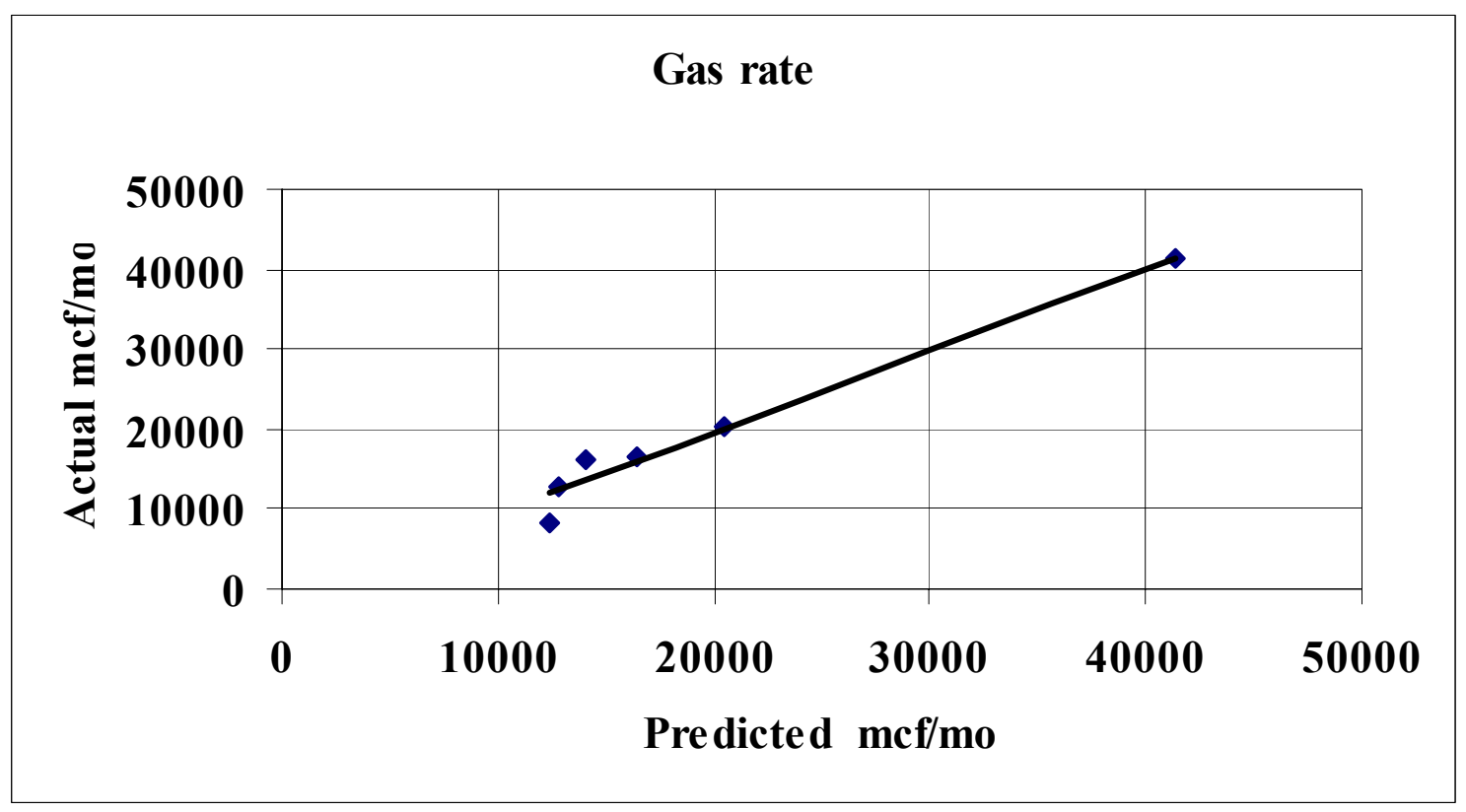

Fig. 50. Sum and average neural network predictions vs. actual initial gas rate. 


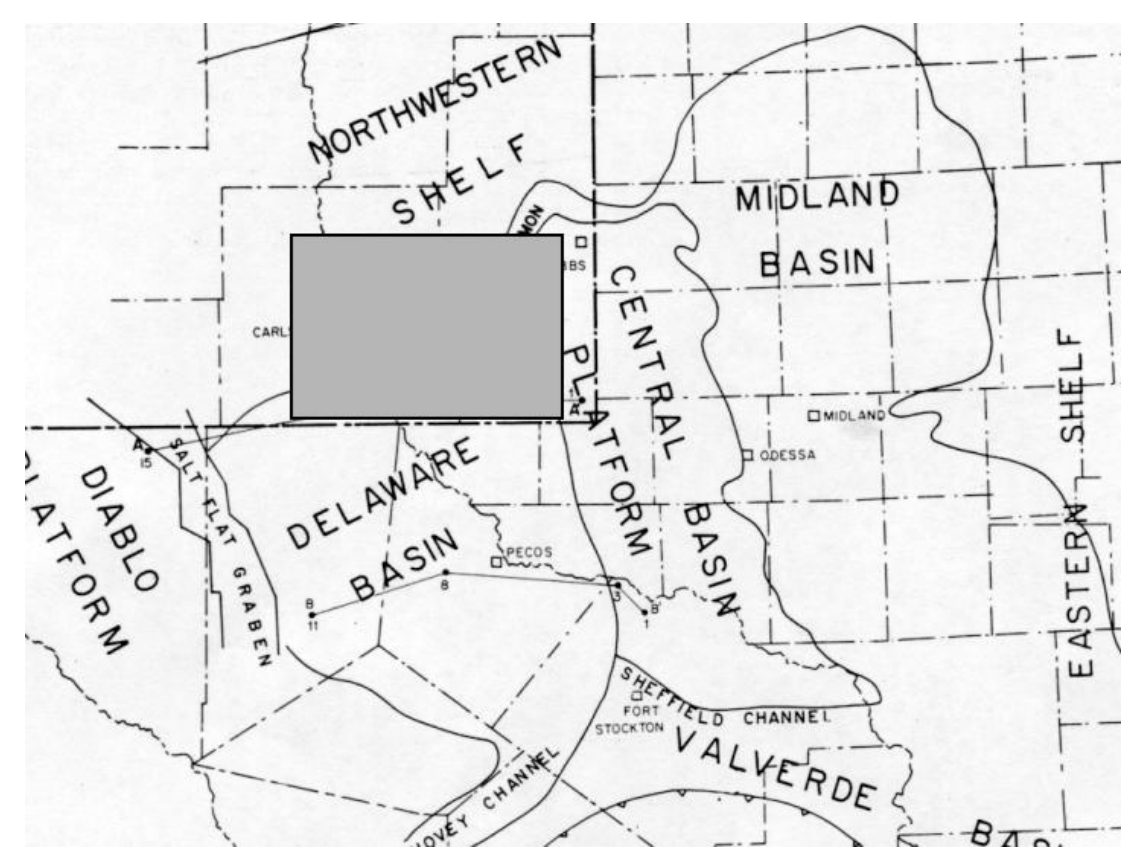

Figure 51. Location of project area in relation to Delaware Basin and other geologic elements, Permian Basin area southeast New Mexico and west Texas. Geologic elements modified from Hills (1984).

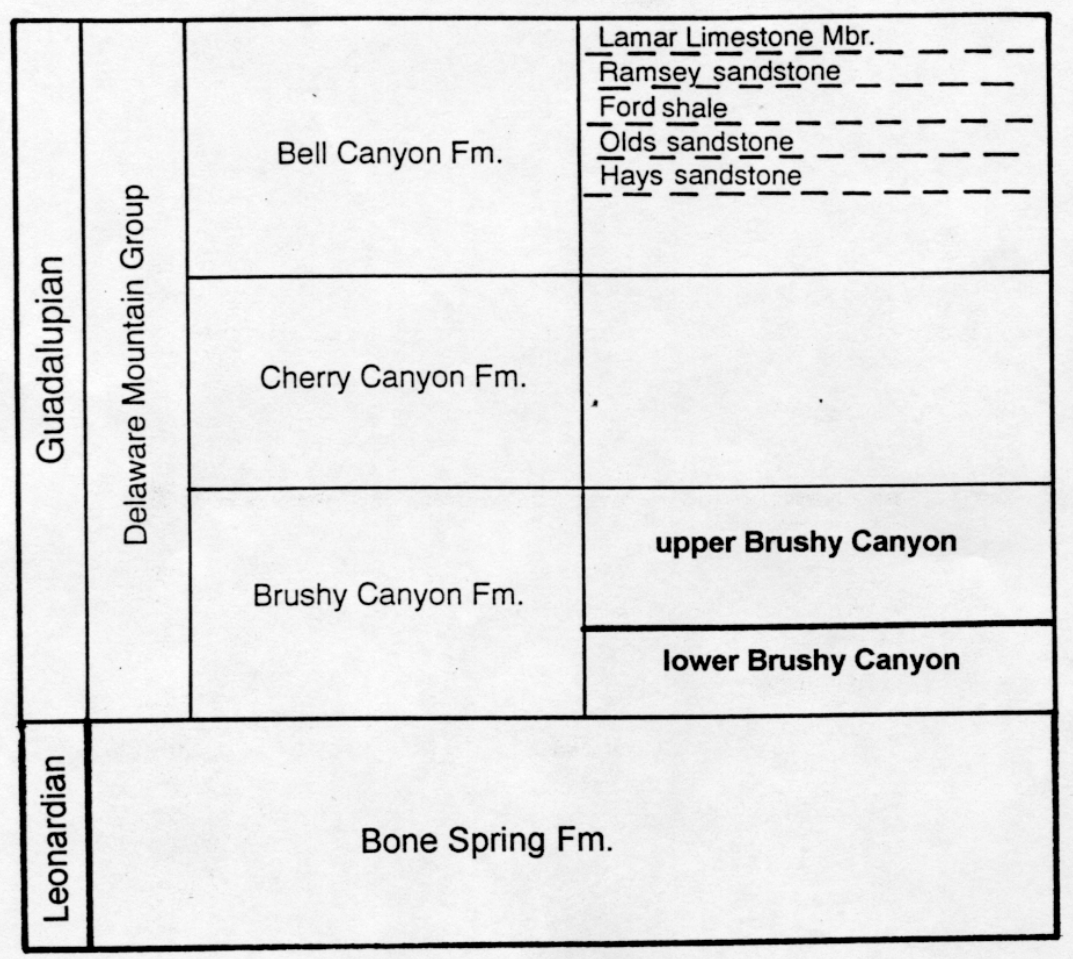

Figure 52. Stratigraphic chart of Delaware Mountain Group. Compiled from Payne (1976), Grauten (1979), Harms and Williamson (1988), and Montgomery et al. (1999). 


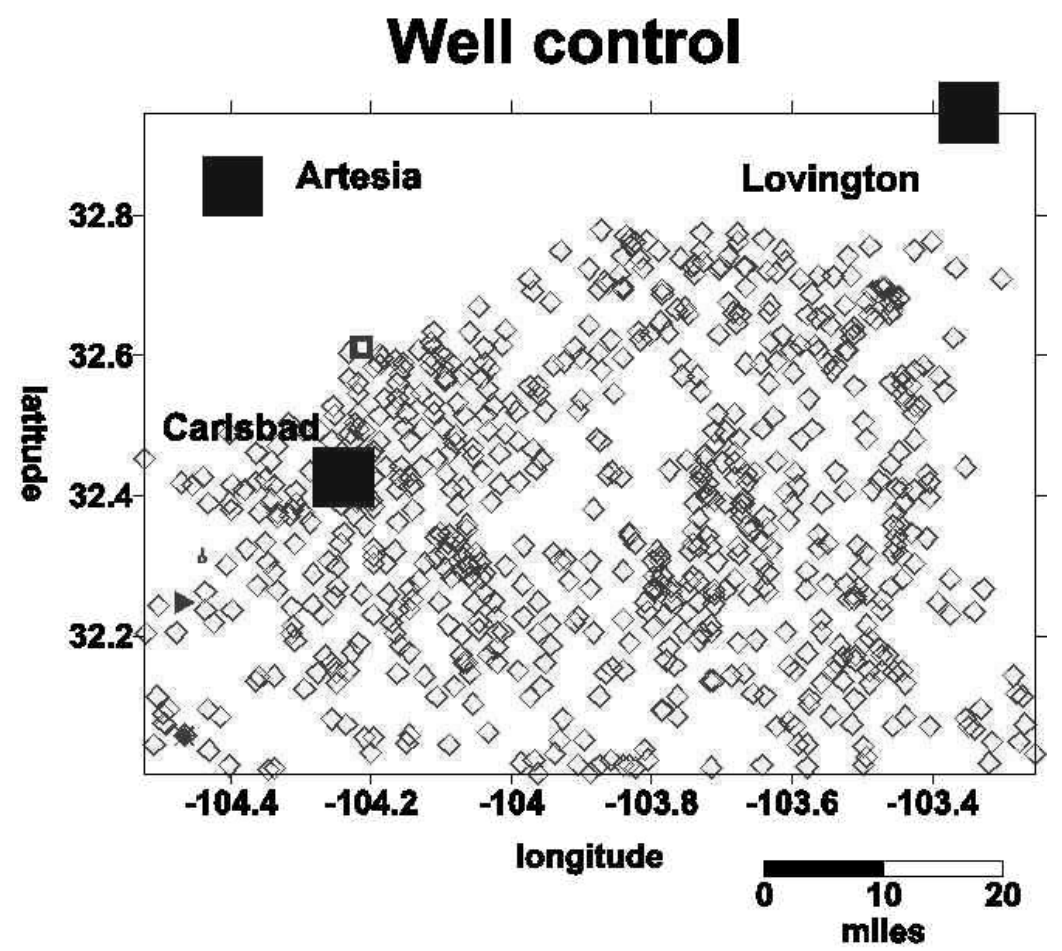

Figure 53. Location of well data control points used in the Brushy Canyon part of this project. See Figure 511 for map location.

\section{Bone Spring structure}

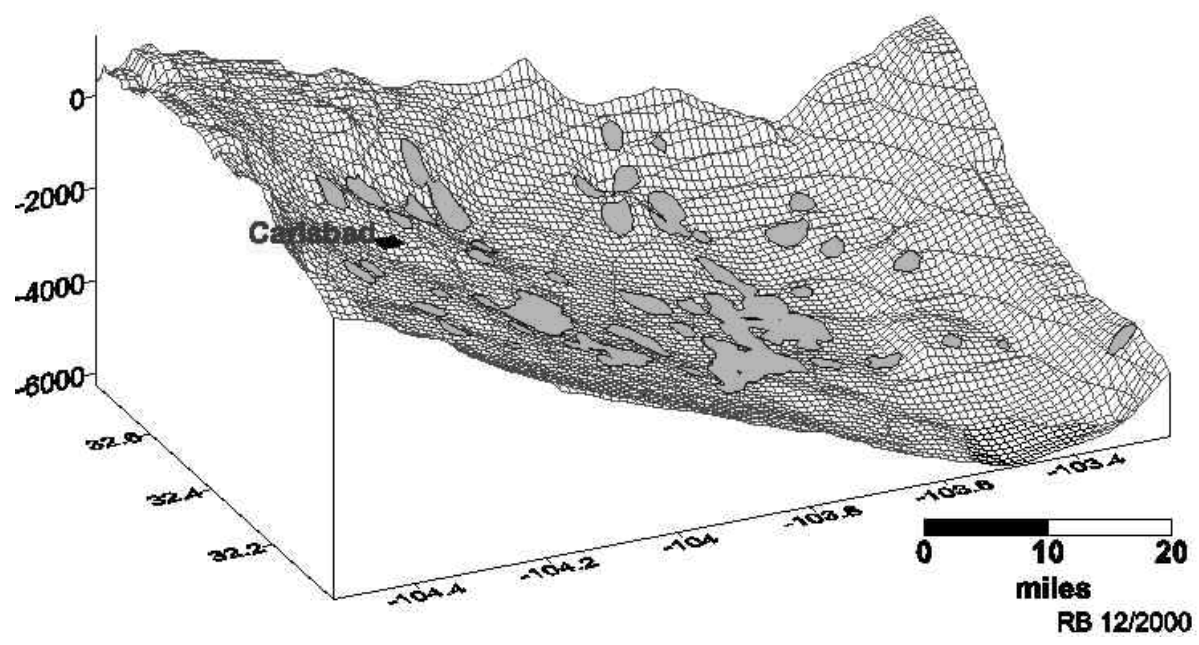

Figure 54. Three-dimensional view of structure on Bone Spring Formation and location of oil pools (in green) productive from the lower part of the Brushy Canyon Formation. 


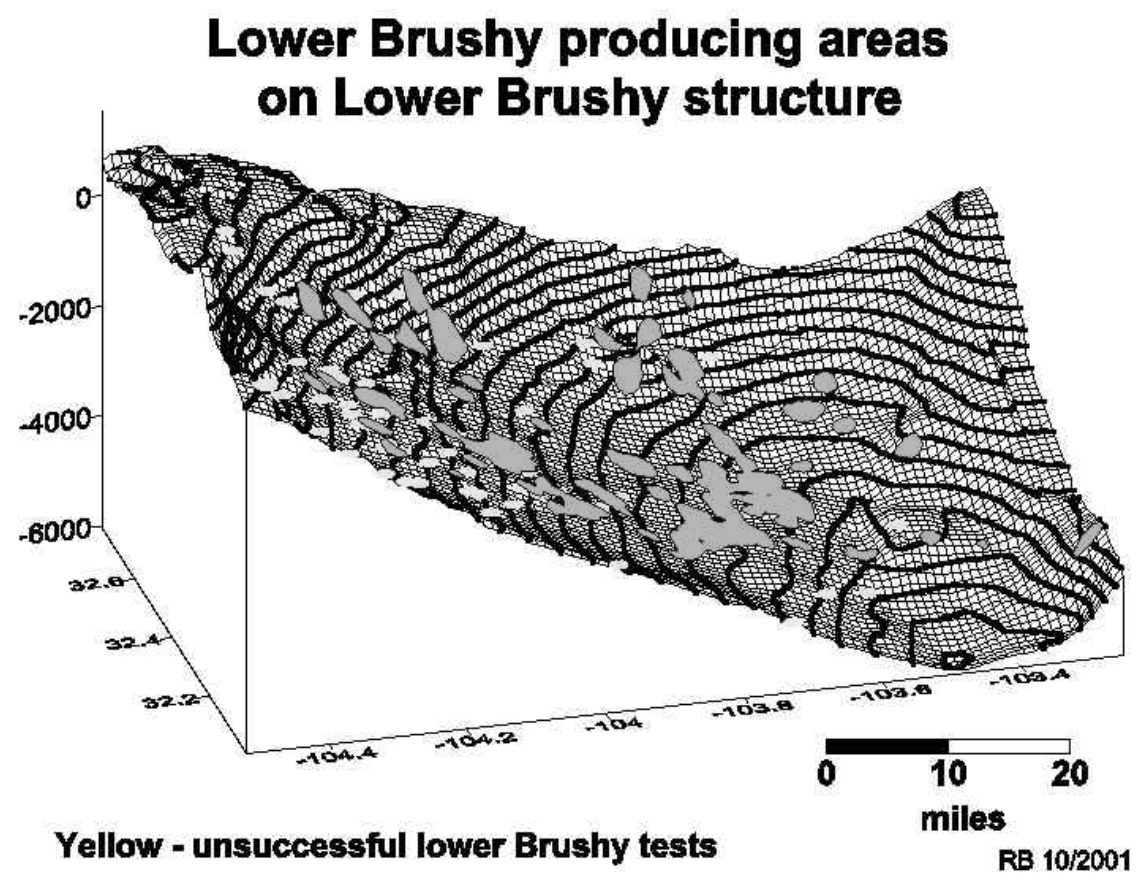

Figure 55. Three-dimensional view of structure on lower Brushy Canyon Formation and location of oil pools (in green) productive from the lower part of the Brushy Canyon Formation.

\section{Lower Brushy Isopach}

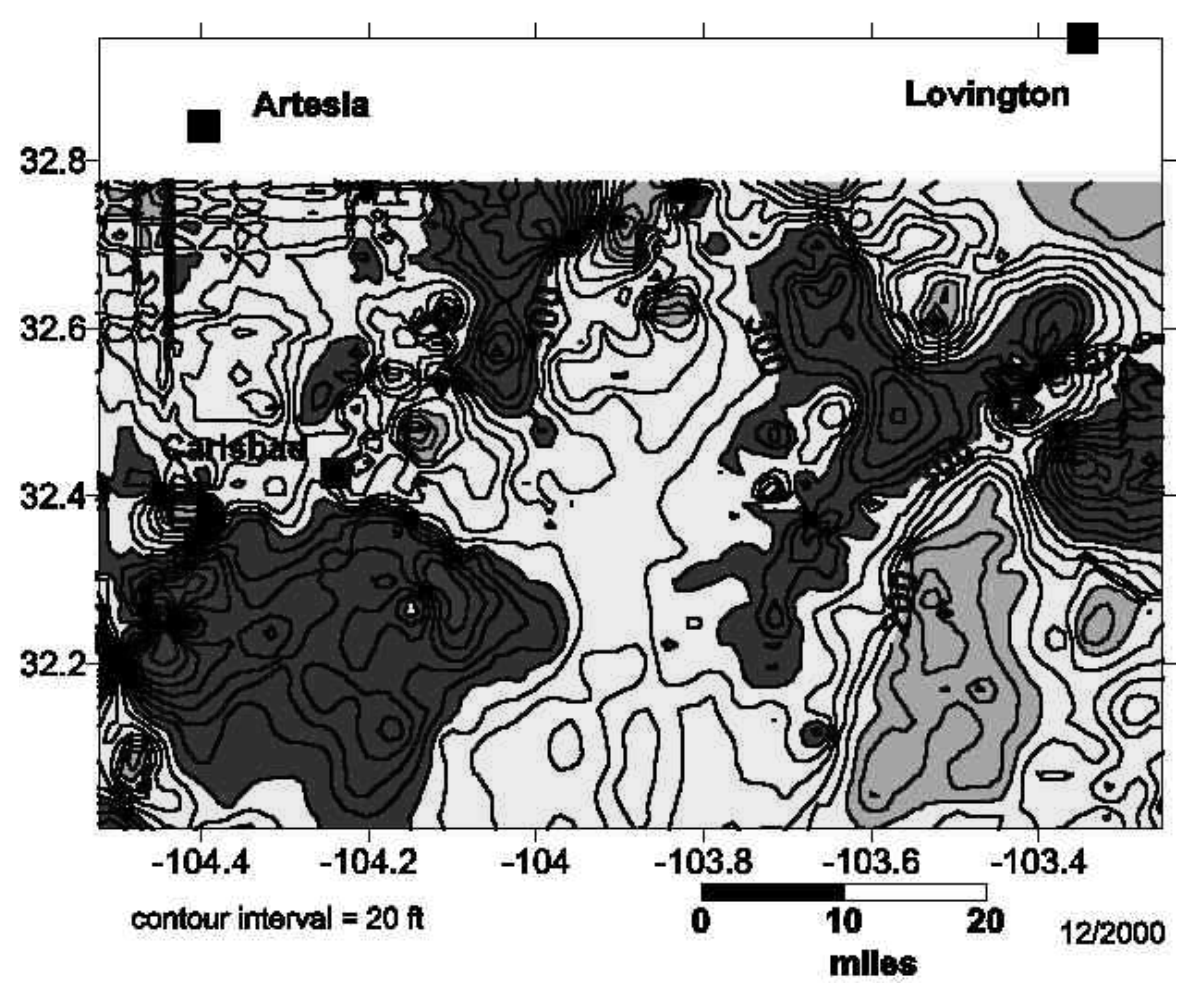

Figure 56. Isopach map of lower Brushy Canyon Formation. 


\section{Lower Brushy production, unsuccessful}

tests and percentage ss with $\mathrm{PHI}>15 \%$

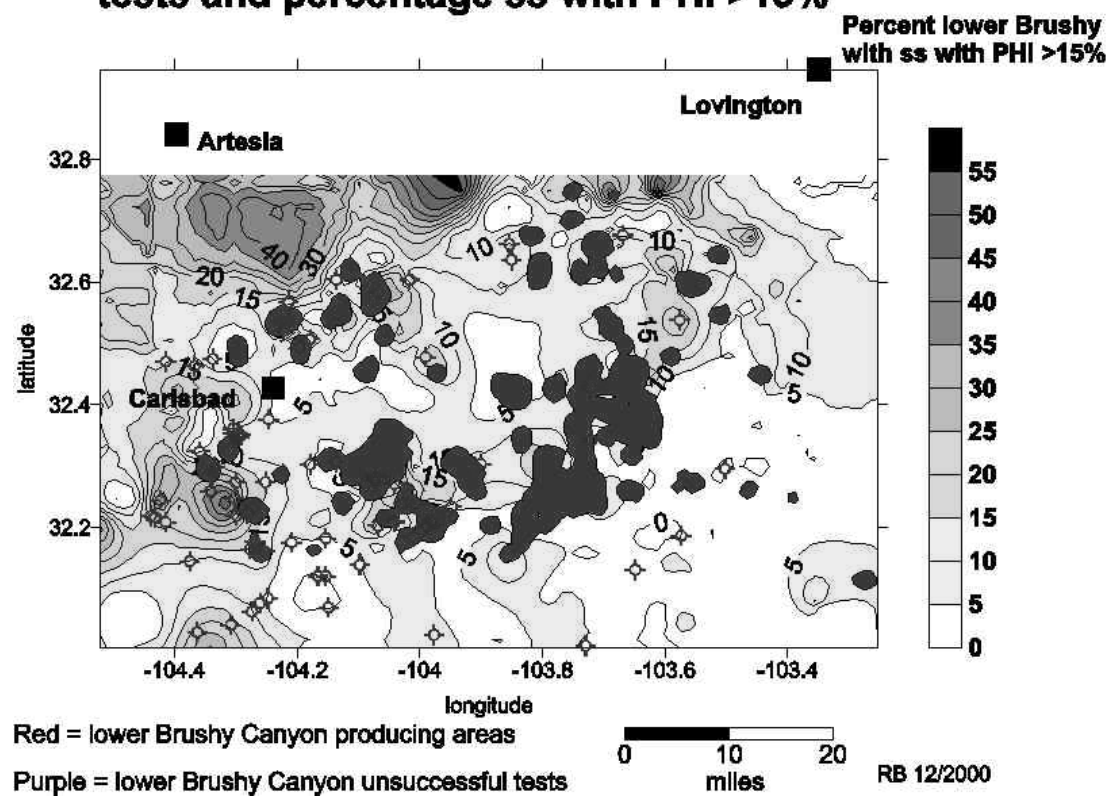

Figure 57. Areas productive from lower Brushy Canyon, net thickness of lower Brushy Canyon sandstones with porosity $>15 \%$, and wells that unsuccessfully tested the lower Brushy Canyon.

\section{Lower Brushy Canyon thickness ss with $>10 \% \mathrm{PHI}$ and lower Brushy production}

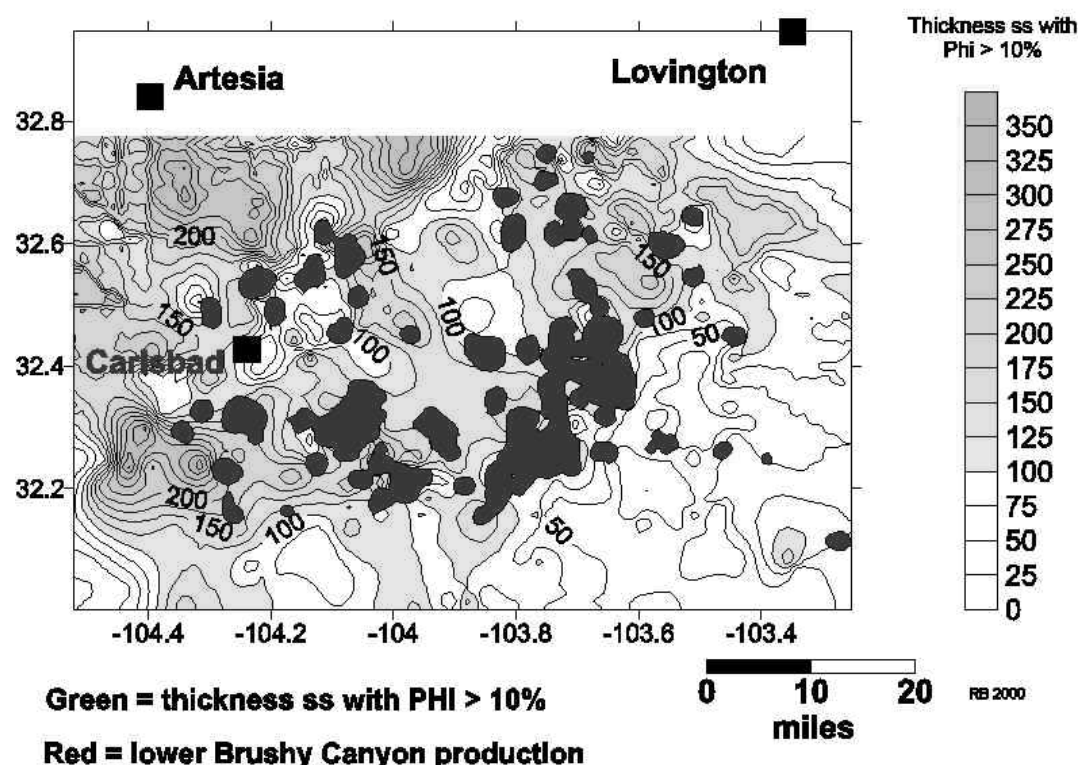

Figure 58. Areas productive from lower Brushy Canyon and net thickness of lower Brushy Canyon sandstones with porosity $>10 \%$. 


\section{Lower Brushy PHI>15\% on Bone Spring structure}

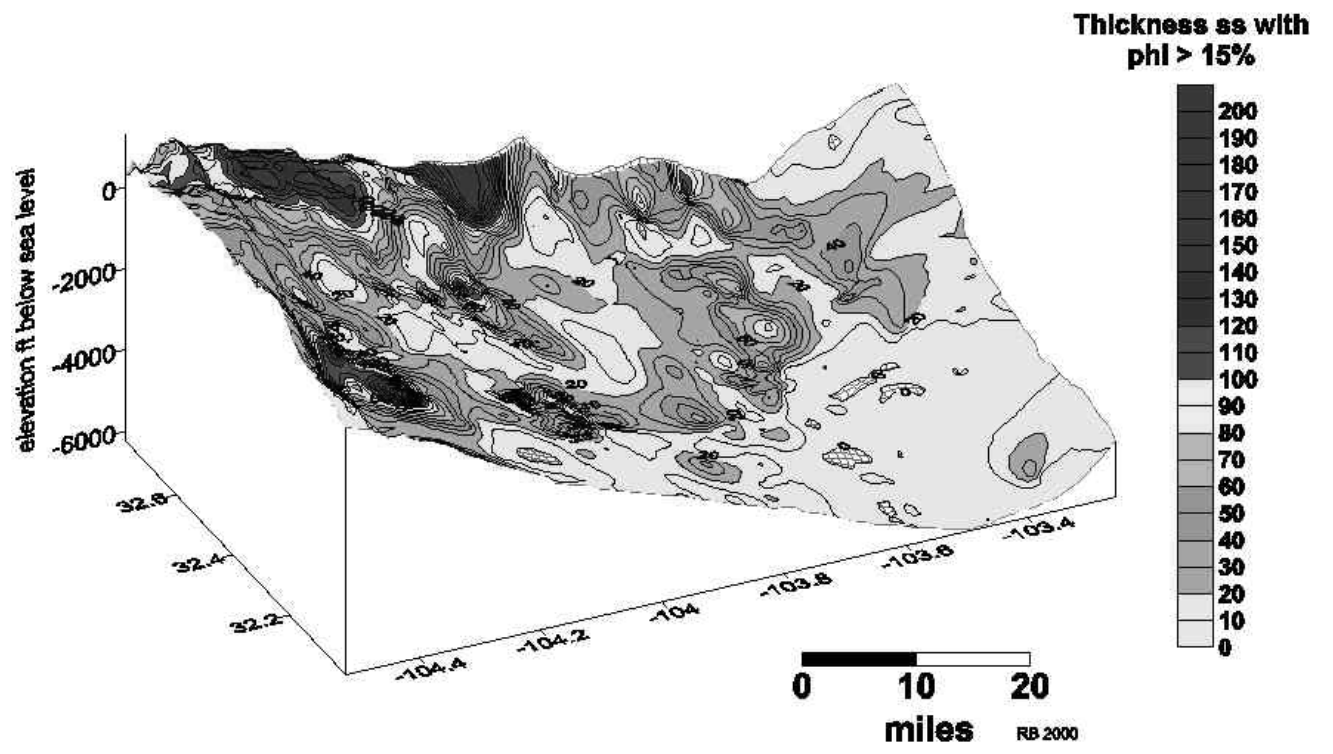

Figure 59. Net thickness of lower Brushy Canyon sandstones with porosity $>15 \%$ superimposed on 3-D diagram of Bone Spring structure.

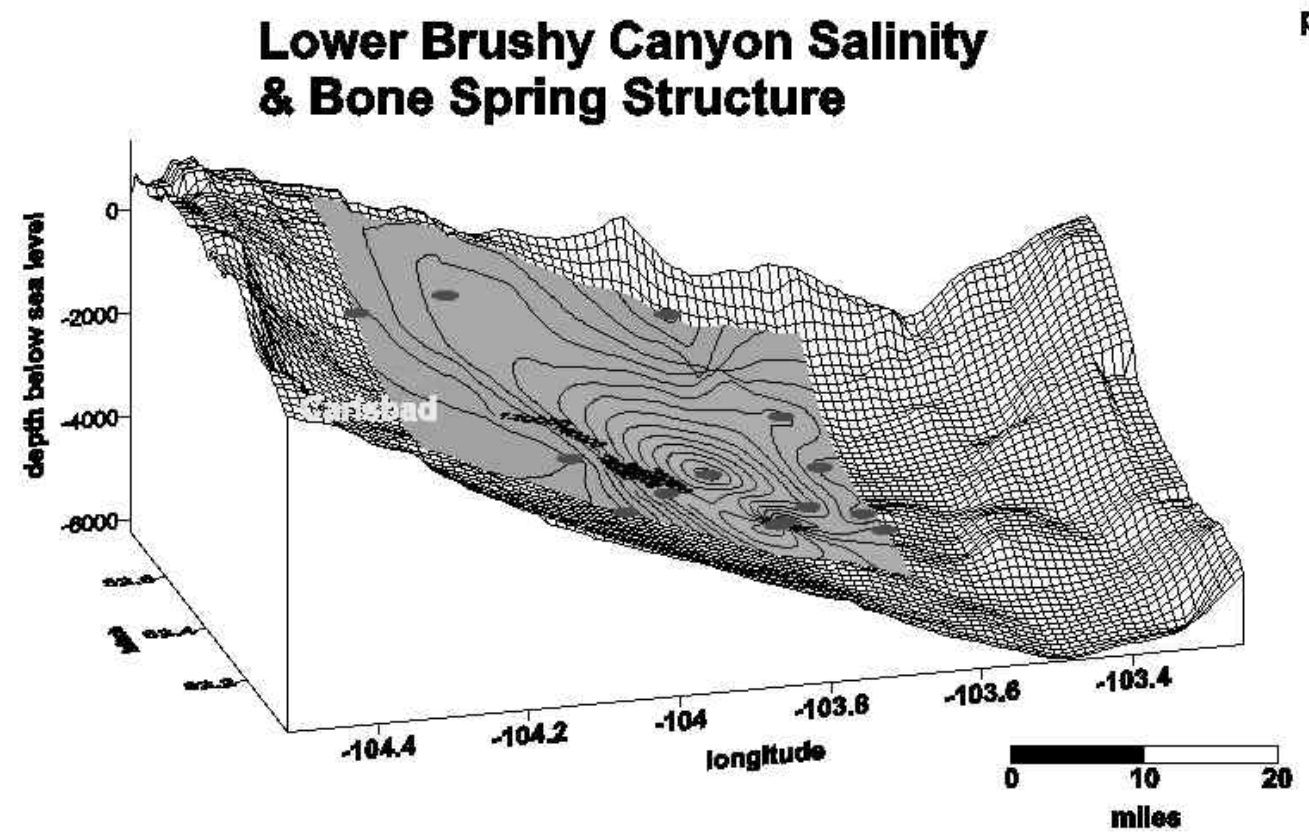

water sallnity ppm chlorldes

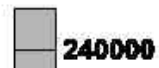

230000

220000

210000

200000

190000 180000 170000 160000 150000 140000 130000 120000 110000 100000 90000

Figure 60. Salinity of produced Brushy Canyon waters superimposed on 3-D diagram of Bone Spring structure. 


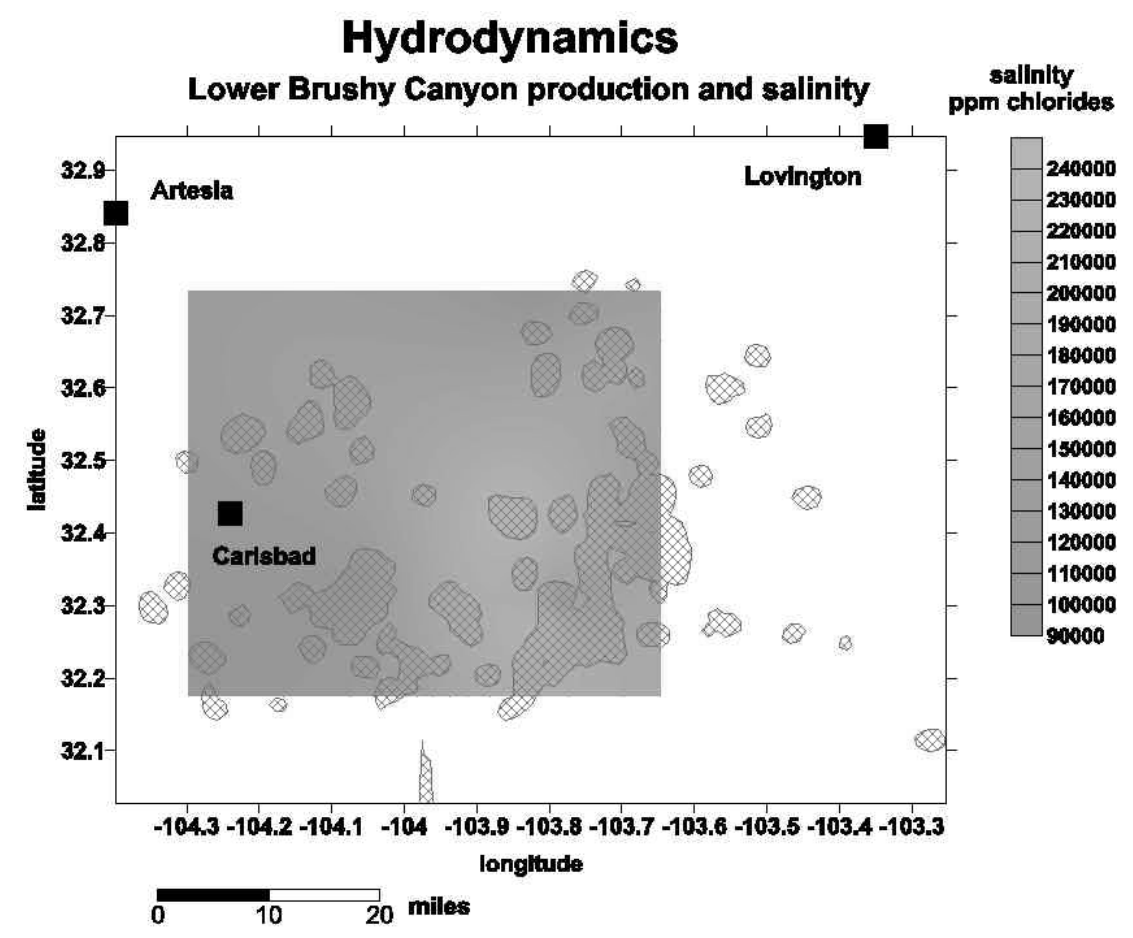

Figure 61. Salinity of produced lower Brushy Canyon waters and areas productive from lower Brushy Canyon.

Lower Brushy production and Lower Brushy TOC

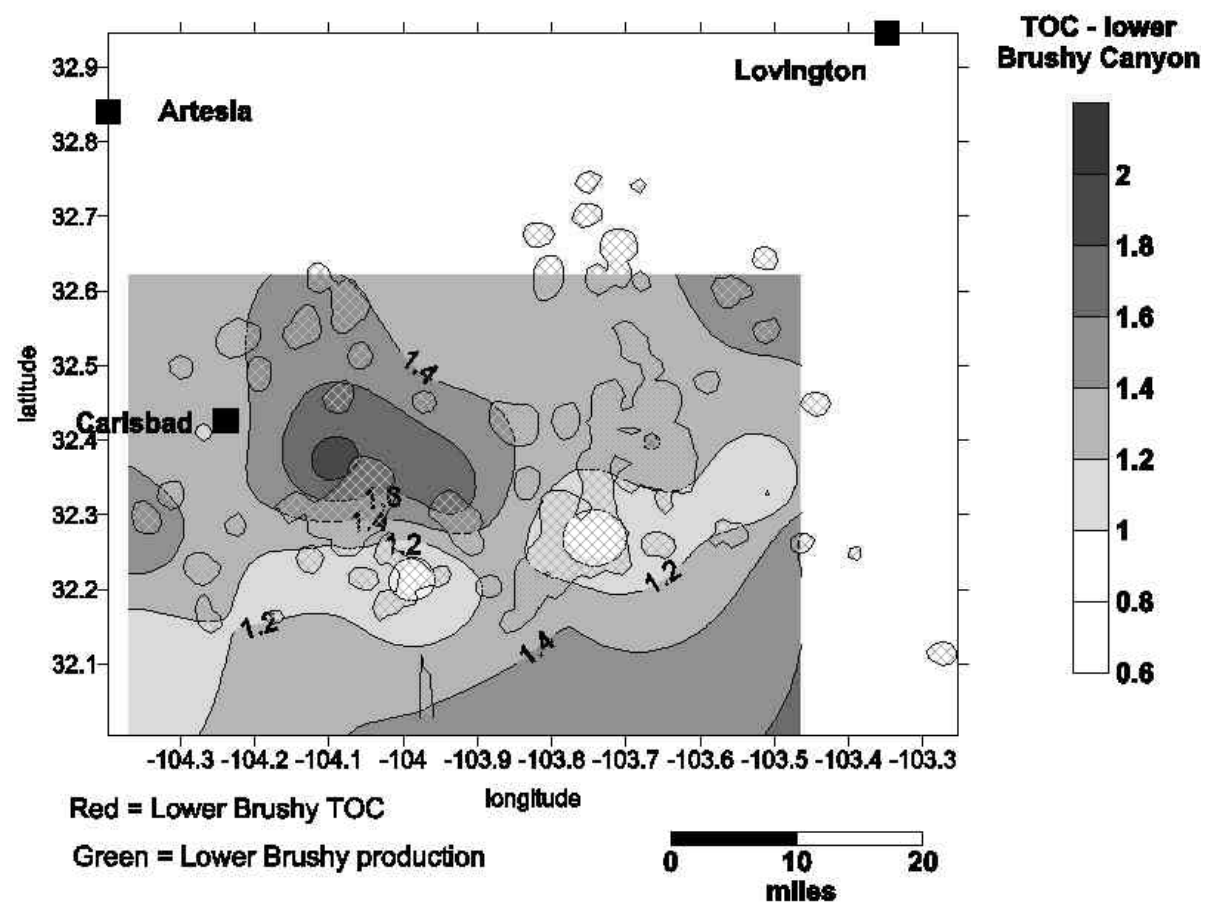

Figure 62. Total organic carbon content, in weight percent, of lower Brushy Canyon source rocks. 


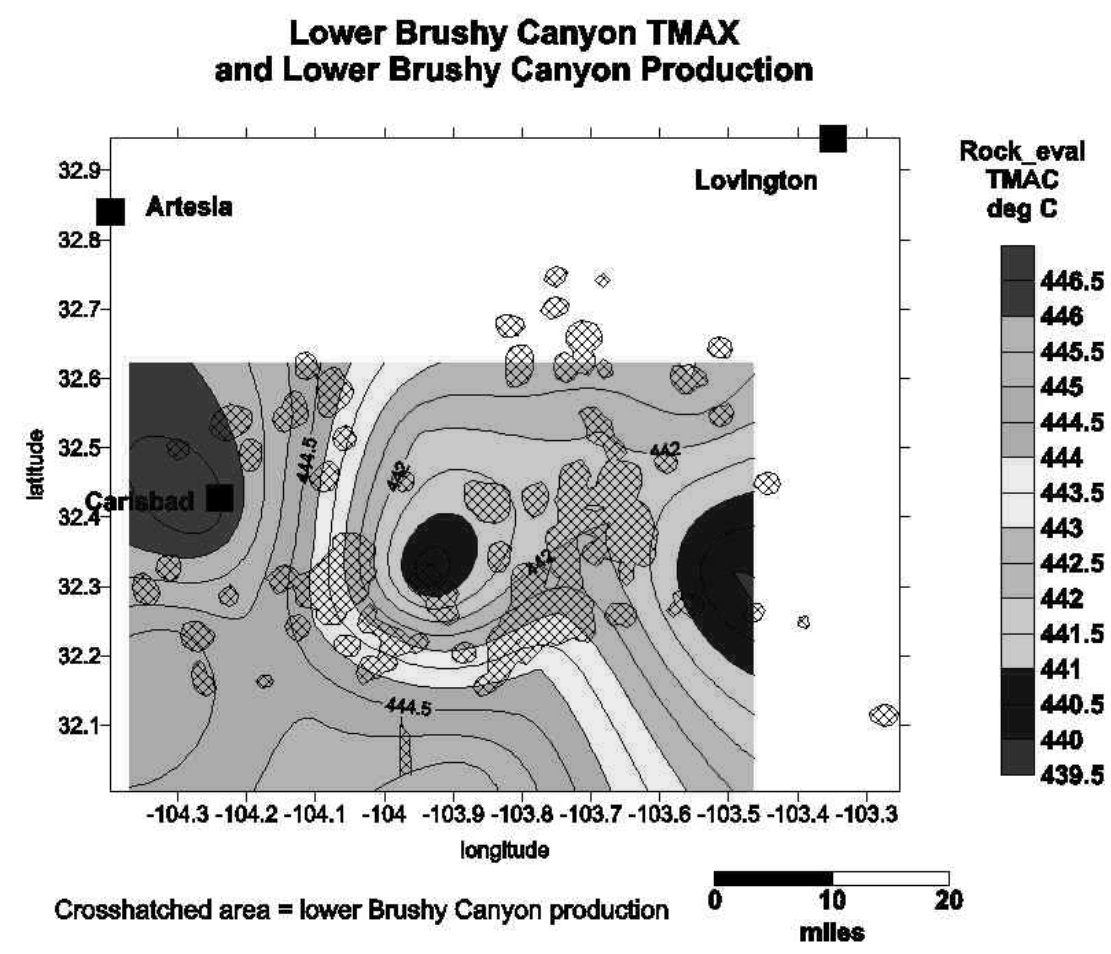

Figure 63. Thermal maturity of lower Brushy Canyon source rocks as determined by Rock-eval TMAX values and areas productive from lower Brushy Canyon.

\section{Lower Brushy Canyon OII Gravlty}

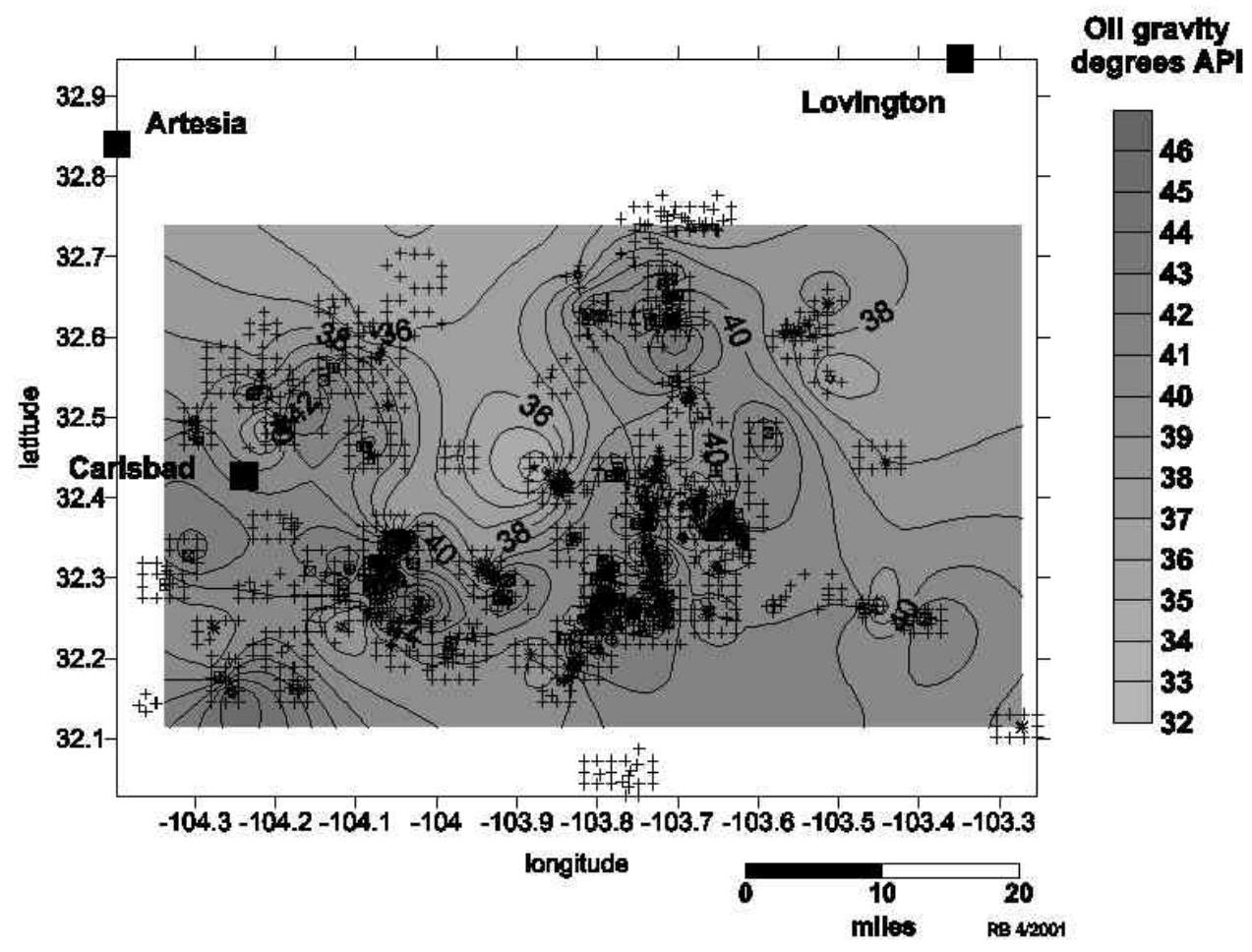

Figure 64. API gravity of lower Brushy Canyon oils. 


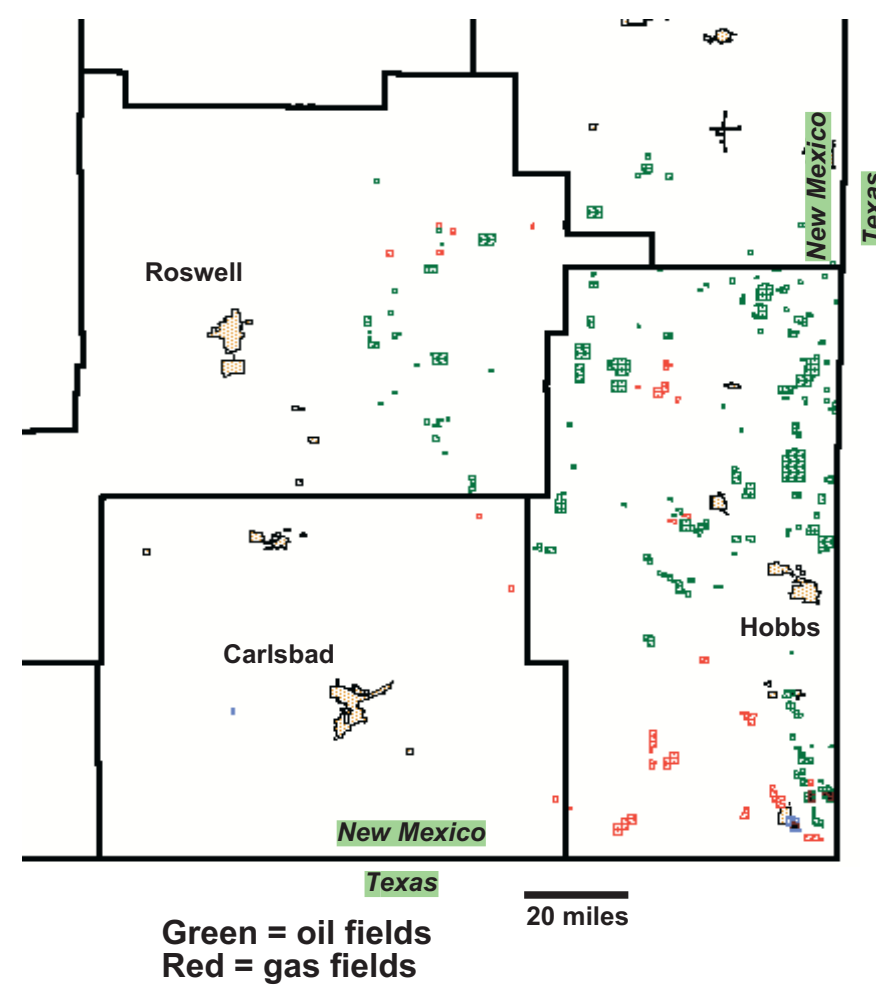

Figure 65. Oil and gas fields productive from Siluro-Devonian carbonates reservoirs in southeast New Mexico.

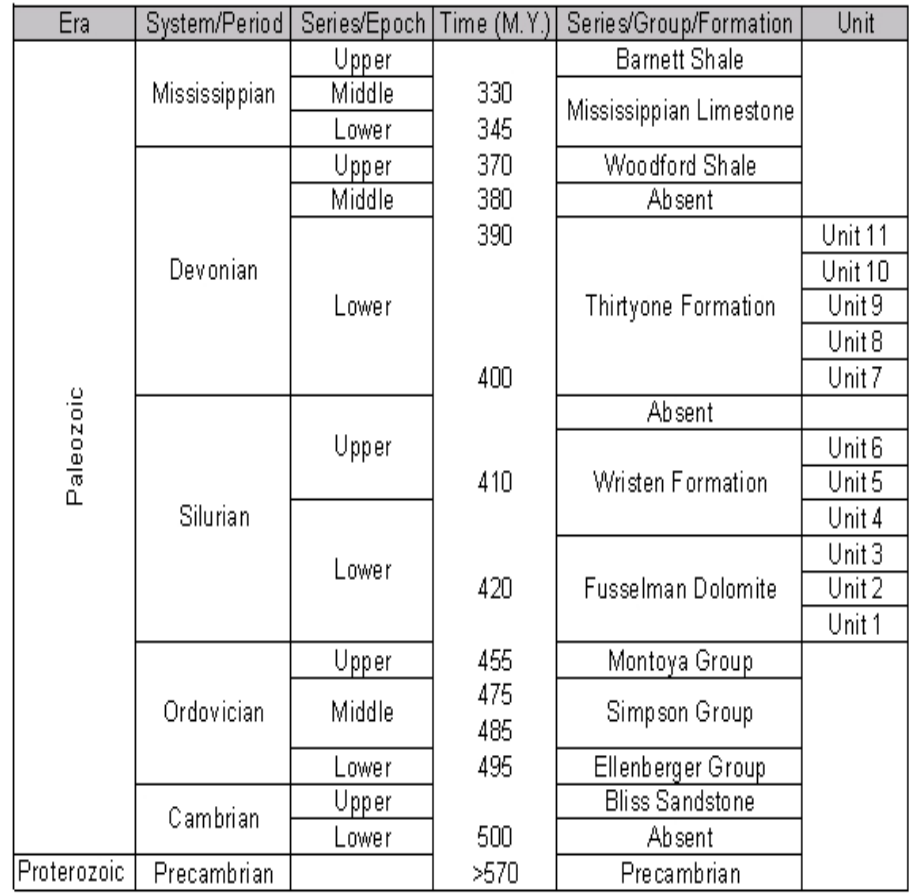

Figure 66. Stratigraphic column of lower Paleozoic strata in southeast New Mexico. Production in the Siluro-Devonian section is obtained from several zones within the Thirtyone, Wristen, and Fusselman Formations. Modified from Hill (1986). 


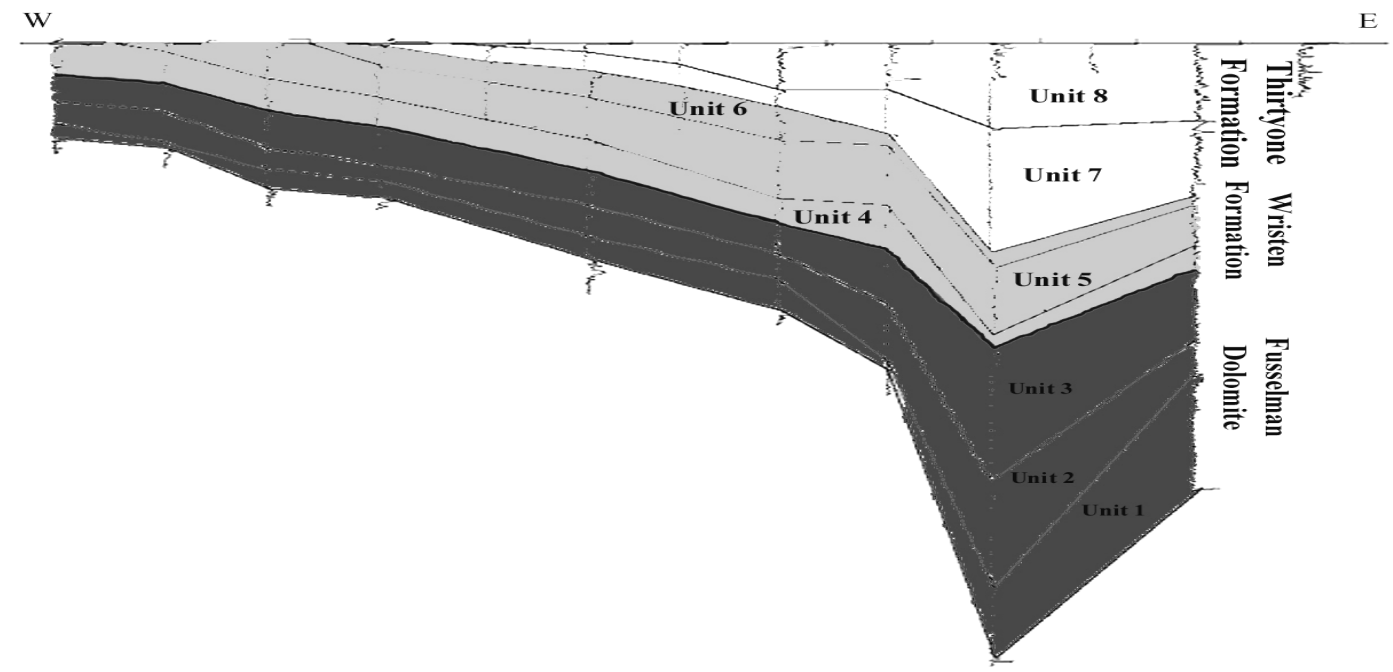

Figure 67. Regional west-east cross section across the Delaware Basin in southeastern New Mexico, showing internal stratigraphy of the Siluro- Devonian carbonate section.

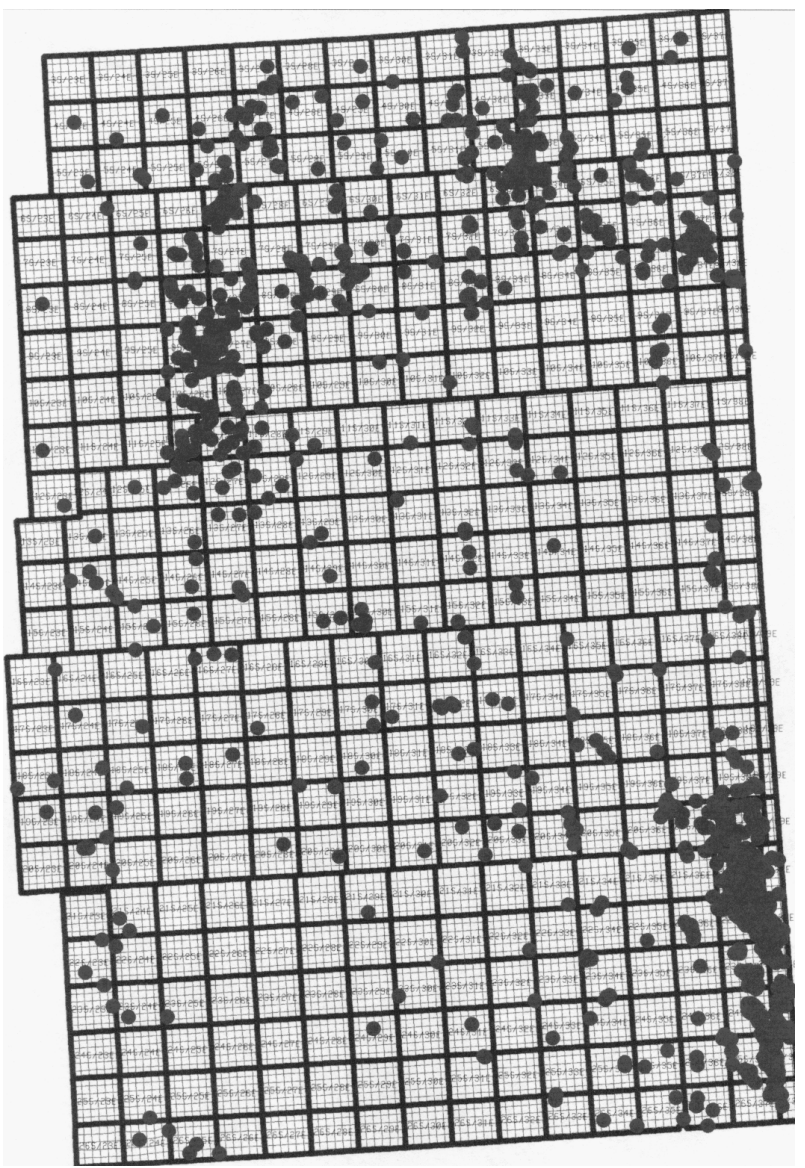

Figure 68. Wells penetrating sub-Woodford Devonian strata in southeast New Mexico. Logs from 465 of these wells were correlated for this project. 


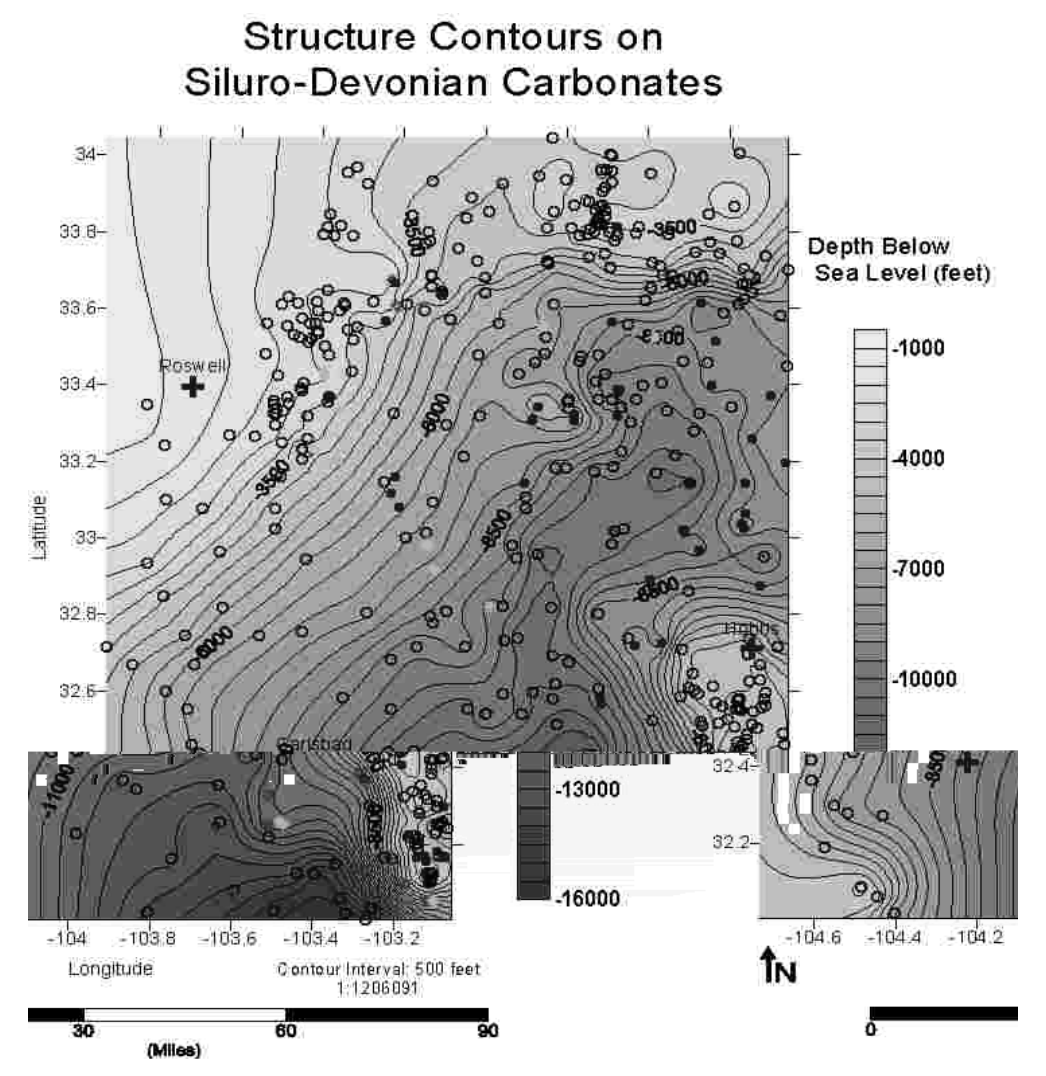

Figure 69. Structure contour map of Siluro-Devonian carbonates.

Thickness of Siluro-Devonian Carbonates

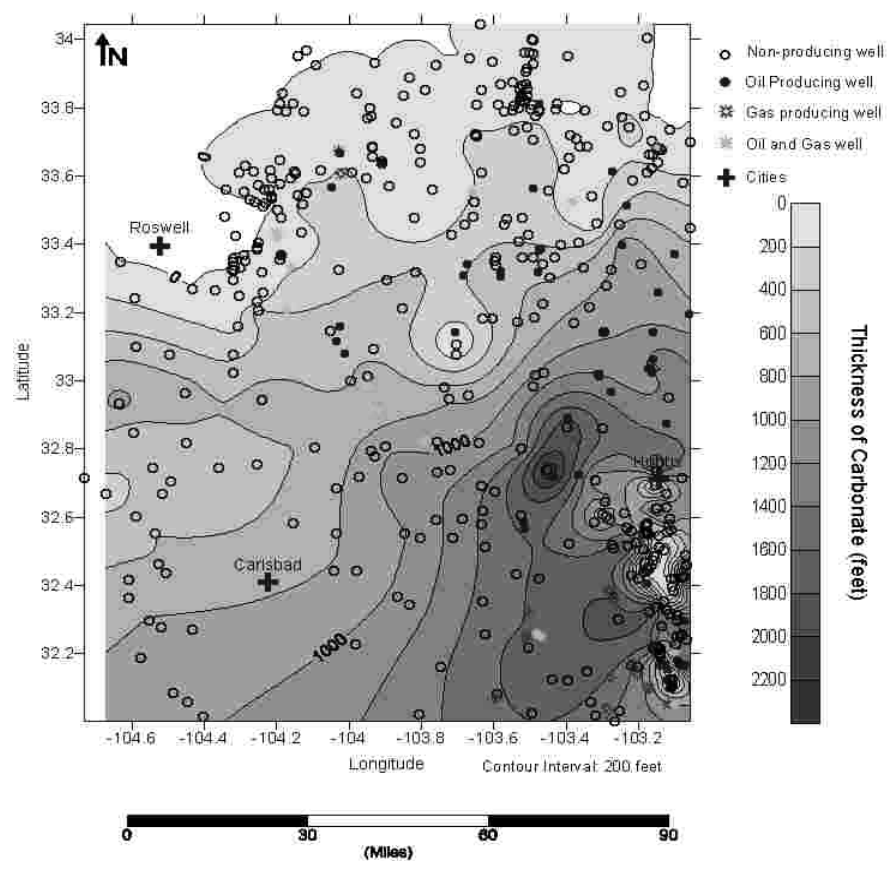

Figure 70. Isopach map of Siluro-Devonian strata in southeastern New Mexico. 


\section{Woodford sub-crop map}

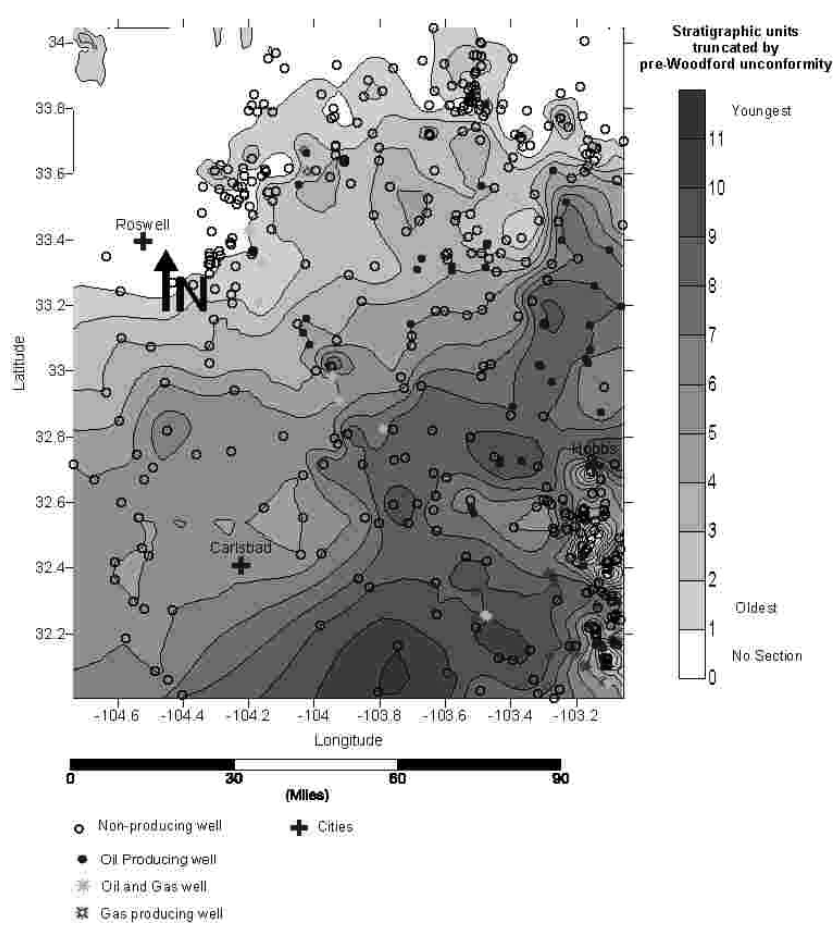

Figure 71. Woodford subcropmap.

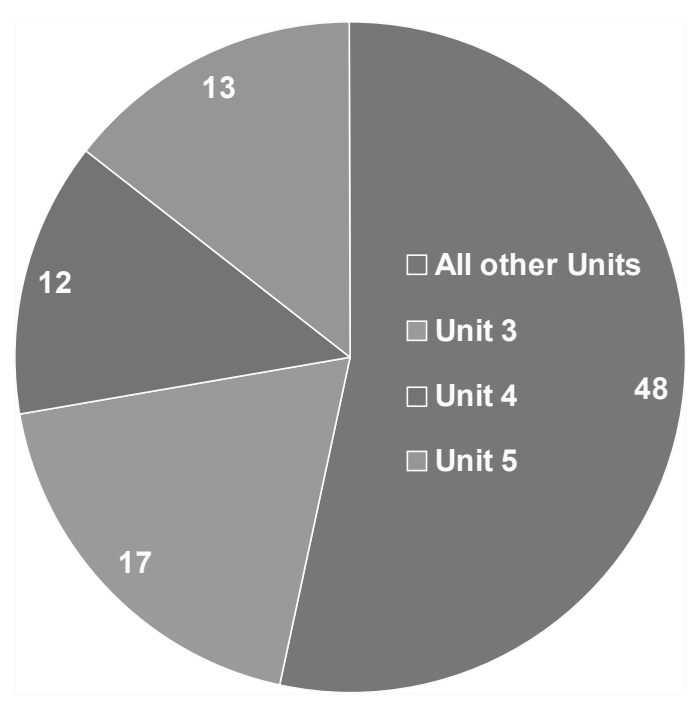

Figure 72. Pie diagram indicating relative number of wells that each SiluroDevonian stratal unit (see Fig. 66) produces. 


\section{Woodford Shale Rock-Eval Productivity Index}

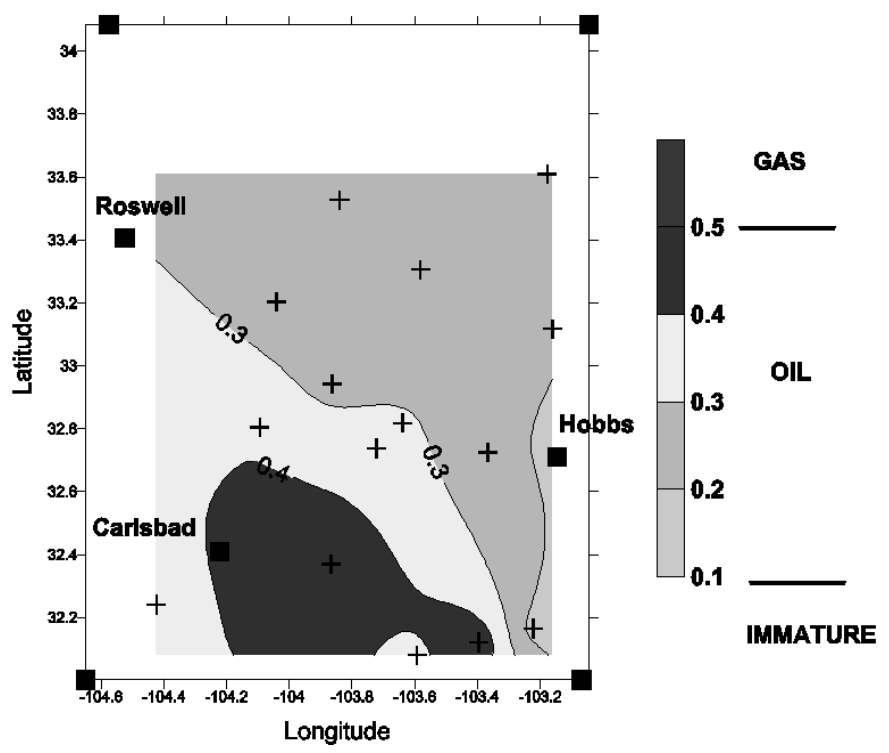

Figure 73. Rock eval productivity index (PI) for the Woodford Shale in southeastern New Mexico. The PI is an indicator of the thermal maturity of the source rock. Note how the Woodford is most mature (in the south-central part of the basin where the underlying Siluro-Devonian carbonates are productive mainly of gas (see Fig. 65).

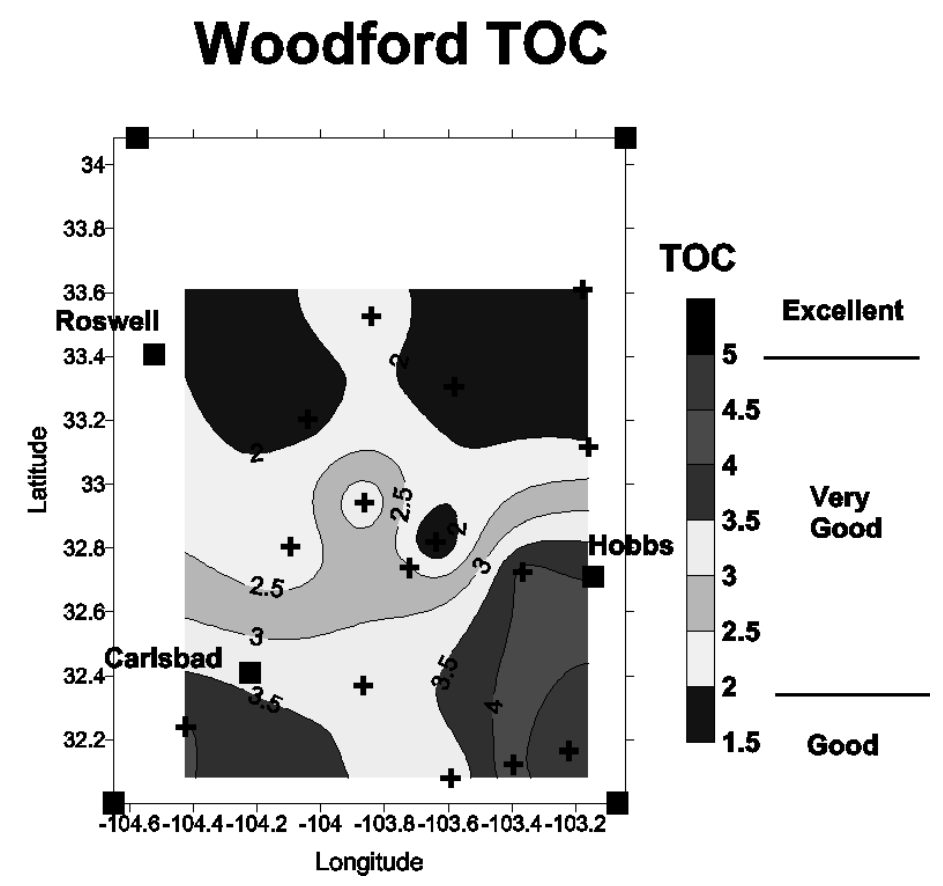

Figure 74. Total organic carbon (TOC) content of Woodford Shale in southeast New Mexico. Note that the Woodford has sufficient TOC for generation of hydrocarbons throughout its extent in southeastern New Mexico. 


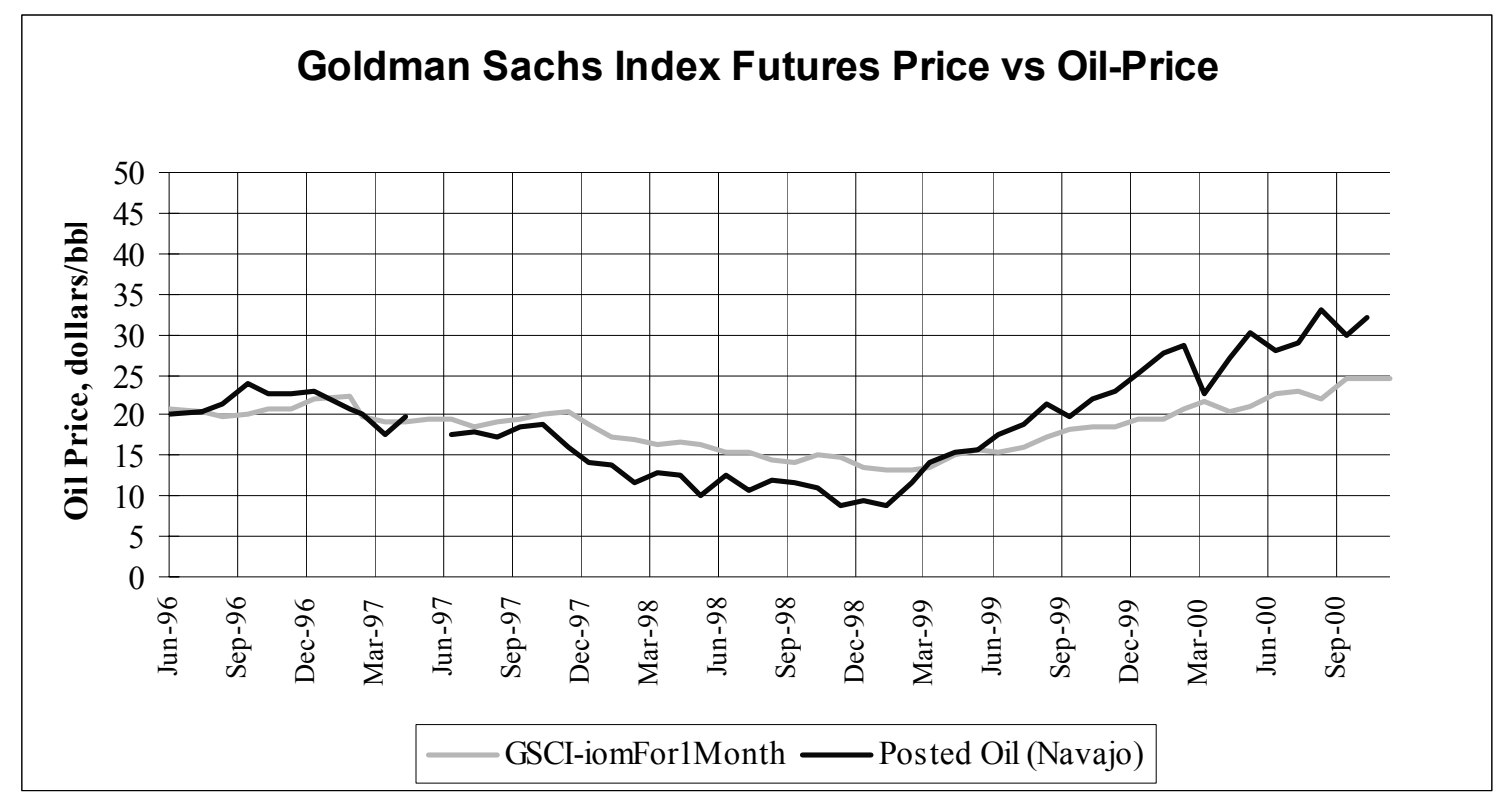

Fig. 75. Actual WTI oil price vs.30-day future price. Time format.

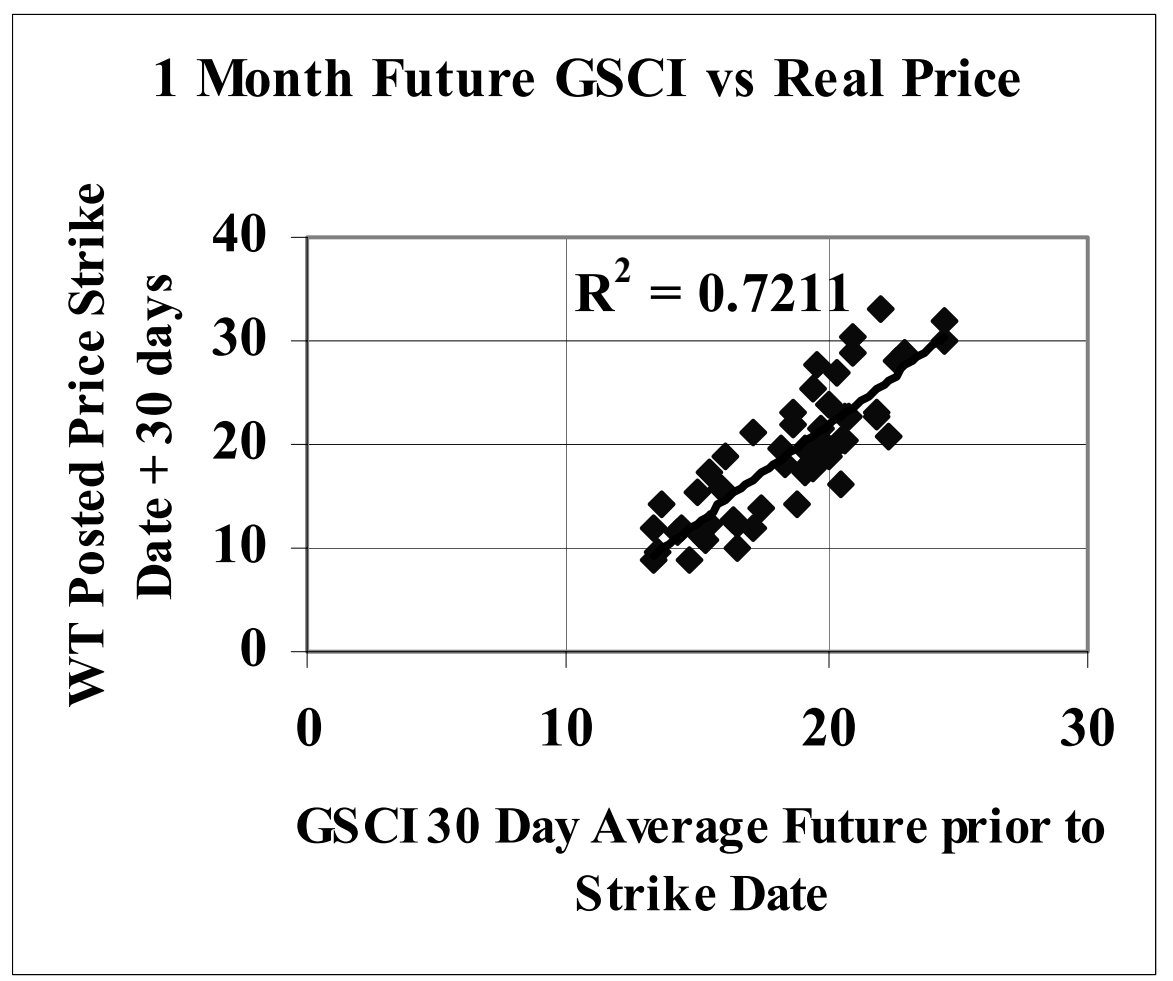

Fig. 76. Actual WTI oil price vs. 30-day future price. Crossplot format. 


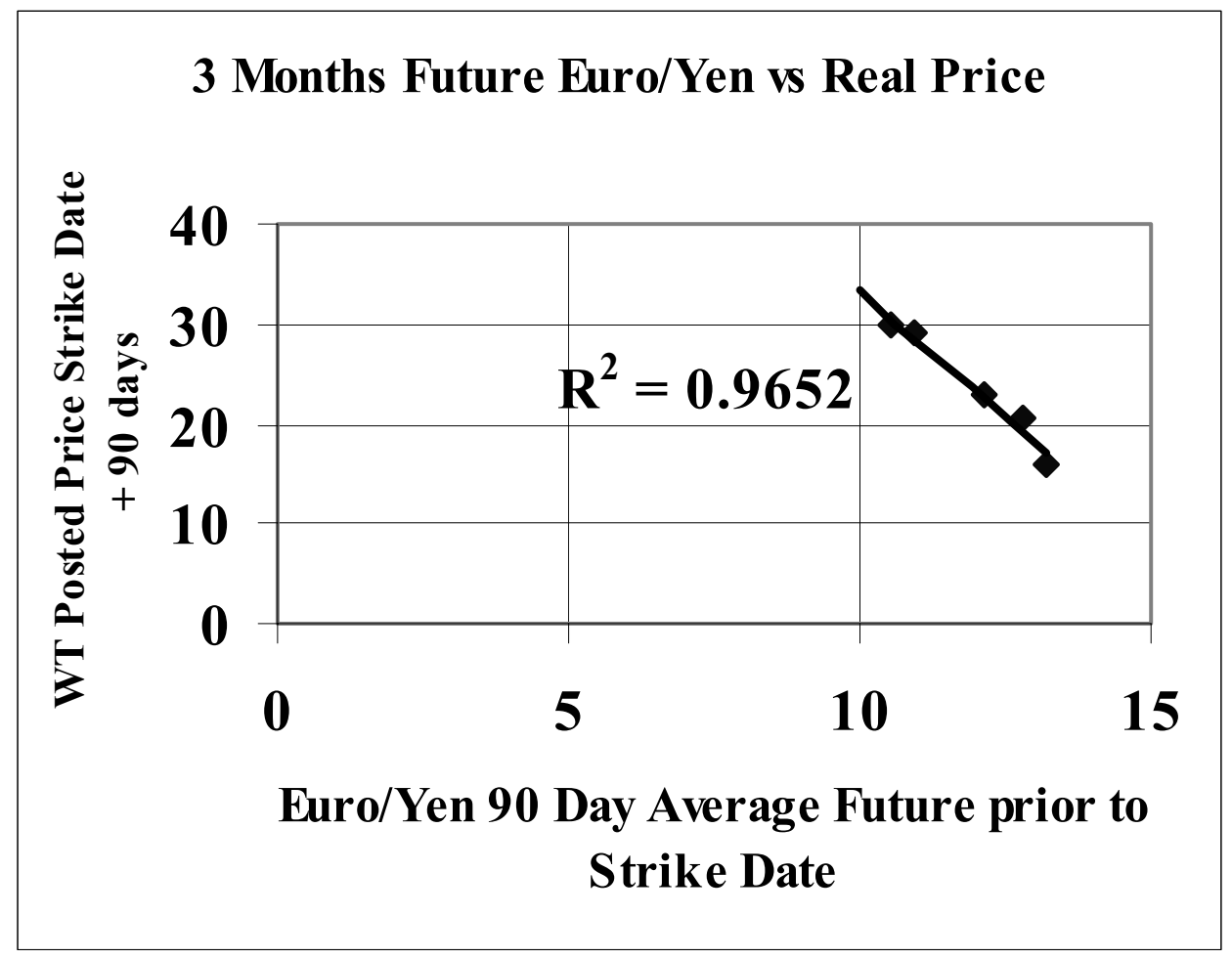

Fig. 77. Actual WTI oil price vs. Euro/Yen 90-day future price. 